\title{
BRAIDED ZESTING AND ITS APPLICATIONS
}

\author{
COLLEEN DELANEY, CÉSAR GALINDO, JULIA PLAVNIK, ERIC C. ROWELL, \\ AND QING ZHANG
}

\begin{abstract}
We give a rigorous development of the construction of new braided fusion categories from a given category known as zesting. This method has been used in the past to provide categorifications of new fusion rule algebras, modular data, and minimal modular extensions of super-modular categories. Here we provide a complete obstruction theory and parameterization approach to the construction and illustrate its utility with several examples.
\end{abstract}

\section{INTRODUCTION}

Despite recent progress on the classification of braided fusion categories, the general landscape remains largely unexplored. This is partly due to our lack of well-studied constructions. Most come from a few basic classes of fusion categories: subquotients of representation categories of quantum groups at roots of unity, representations of quasi-Hopf algebras, bimodule categories over finite index finite depth subfactors, planar algebras, and near-group categories. There are a few inter-related tools for obtaining new braided fusion categories from old such as the Drinfeld center construction, graded extensions by finite groups $G, G$-equivariantization $/ G$-de-equivariantization, symmetry gauging, and Deligne products. In this paper, we develop another recent construction known as zesting.

Zesting first appeared as a construction technique in [5] for the purpose of categorifying a mysterious rank 10, dimension 36 fusion rule algebra, with fusion rules reminiscent of, but distinct from, those of $S U(3)_{3}$. It was recognized that the fusion rules could be obtained from those of $S U(3)_{3}$ by rearranging them slightly, via a twisting of the tensor product.

The basic goal of zesting is to define new braided fusion categories from a given $A$-graded braided fusion category $\mathcal{C}=\bigoplus_{a \in A} \mathcal{C}_{a}$ by defining a new tensor product $X_{a} \stackrel{\lambda}{\otimes} Y_{b}:=\left(X_{a} \otimes Y_{b}\right) \otimes \lambda(a, b)$, where $X_{a} \in \mathcal{C}_{a}$ and $Y_{b} \in \mathcal{C}_{b}$ are simple objects in

2000 Mathematics Subject Classification. 16W30, 18D10, 19D23.

The authors gratefully acknowledge the support of the American Institute of Mathematics, where this collaboration was initiated. C.G would like to thank the hospitality and excellent working condition of the Department of Mathematics at the University of Hamburg, where he carried out this research as Fellows of the Humboldt Foundation. J.P was partially supported by US NSF Grants DMS-1802503 and DMS-1917319. E.C.R. was partially supported by US NSF Grant DMS-1664359, a Simons Foundation Fellowship, and a Texas A\&M Presidential Impact Fellowship. Part of this research was carried out while CD, JP, ER and QZ participated in a semester-long program at MSRI, which is partially supported by NSF grant DMS-1440140. We thank Richard Ng for useful discussions. 
their corresponding graded components and $\lambda(a, b) \in \mathcal{C}_{e}$ is an invertible object in the trivial component.

As one might expect, there are obstructions to $(\mathcal{C}, \stackrel{\lambda}{\otimes}, \mathbf{1})$ admitting the structure of a monoidal category for a given choice of $\lambda: A \times A \rightarrow \mathcal{C}_{p t} \cap \mathcal{C}_{e}$. That is, it is not immediate that associativity morphisms satisfying pentagons exist, and when these obstructions vanish there are inequivalent choices of associativity morphisms. Fixing a particular such associative zesting one can further investigate whether the category admits a braiding, which leads to more obstructions and choices. Such a braided structure is called a braided zesting. Finally, for a fixed braided zesting of a ribbon fusion category we may look for a balancing structure, which we call a twist zesting in general and a ribbon zesting in the case the twist has the ribbon property.

We hasten to point out that our associativity, braiding and twist choices for a zesting are assumed to only depend on the grading group $A$, the pointed subcategory of the trivial component, and the structures already present in $\mathcal{C}$. It follows that the trivial component of $\mathcal{C}$ and that of any zesting of $\mathcal{C}$ are equivalent as fusion categories so that a zesting is always an extension (in the sense of [16]) of the trivial component by the group $A$. Moreover, at each step, some of these extensions may fail to be realized by our construction. For example, the Ising categories and the pointed modular categories with fusion rules like $\mathbb{Z} / 4$ are braided $\mathbb{Z} / 2$-extensions of the category of super-vector spaces; however it is not possible to use zesting to construct one from the other, since any zesting of a pointed fusion category remain pointed. On the other hand, we can explicitly obtain both new fusion categories that do not admit braidings and new ribbon braided fusion categories with explicit formulas of their modular data from our approach.

There are two related constructions found in the literature that should be mentioned. The first is gauging [8]: one begins with a modular category $\mathcal{B}$ that admits an action of a finite group $G$ by braided tensor automorphisms, and constructs, assuming certain cohomological obstructions vanish, new modular categories $\mathcal{C}$. The first step is to construct the $G$-crossed braided categories $\mathcal{D}$ with $\mathcal{B}$ as the trivial component (using [16]), and the second takes the $G$-equivariantization, which will be modular. In some cases zestings can be placed in this framework. If $\mathcal{C}$ is modular and $\mathcal{C}_{p t} \cap \mathcal{C}_{e} \cong \operatorname{Rep}(G)$ is Tannakian, we may set $\mathcal{B}=\left(\mathcal{C}_{e}\right)_{G}$ the $G$-de-equivariantization of the trivial component. Then any modular zesting $\tilde{\mathcal{C}}$ will be a $G$-gauging of $\mathcal{B}$. The second construction is related. If $\mathcal{C}_{p t} \cap \mathcal{C}_{e} \cong \operatorname{Rep}(G)$ as above, we may construct new categories as tensor products over $G$ by condensing the diagonal algebra in $\mathcal{C} \otimes \operatorname{Rep}\left(D^{\omega} G\right)$.

While both of these constructions have the advantage of providing various structures from general arguments, zesting has several key features that these do not: 1) one has the fusion rules at the outset 2) we produce new categories that are not necessarily modular or even braided 3) it depends only on essentially cohomological choices and 4) we have explicit formulas for their modular data.

In a bit more detail, the construction goes as follows. For a fixed $A$-graded ribbon category $\mathcal{C}$ where $A=U(\mathcal{C})$ is the universal grading: 
(1) Fix a normalized 2-cocycle $\lambda \in Z^{2}\left(A, \operatorname{Inv}\left(\mathcal{C}_{e}\right)\right)$.

(2) Find a 3-cochain $\lambda \in C^{3}\left(A, \mathbb{k}^{\times}\right)$satisfying the second associative zesting constraint, if possible (see Figure 21). The set of all associative zestings form a torsor over $H^{3}\left(A, \mathbb{k}^{\times}\right)$for the chosen 2-cocycle.

(3) For a fixed associative zesting, a braided zesting is determined by a cochain $c \in C^{2}\left(A, \mathbb{k}^{\times}\right)$such that $t(a, b):=c(a, b) \operatorname{id}_{\lambda(a, b)}$ satisfies the two braided zesting constraints (see Figures 7 and $[8)$. For a fixed such $c(a, b)$ the set of all braided zestings forms a torsor over the group of bicharacters of $A$.

(4) For a fixed braided zesting of a braided fusion category $\mathcal{C}$ with a twist $\theta$, we may determine all braided twist (ribbon) zestings in terms of a function $f: A \rightarrow \mathbb{k}^{\times}$, as in Corollary [5.3, and all twist (ribbon) zestings form a torsor over the characters of $A / 2 A$.

More general situations can be considered as well, for example, we may choose a different grading group $B$ for $\mathcal{C}$ (i.e., a quotient of $U(\mathcal{C})$ ) and the above is still true provided the image of $\lambda \in Z^{2}\left(A, \operatorname{Inv}\left(\mathcal{C}_{e}\right)\right)$ centralizes the trivial component $\mathcal{C}_{e}$ with respect to the grading $B$. Failing this, we may still develop a theory of zesting, but there are several subtleties that must be addressed in the form of additional choices and more elaborate constraints. Moreover, the general definition of associative zesting does not require a braiding - one may apply it to any fusion category.

Zesting supersedes several known constructions as special cases. If one chooses the trivial 2-cocycle in step (1), the second step is the well-known associativity twist (see, e.g. [21]). If one makes the trivial choice of 2-cocycle and 3-cochain in steps (1) and (2), then the braided zestings are simply modifying the braiding by a bicharacter, which is also well-known. Finally, if one takes the trivial choice in each of steps (1), (2), and (3) for a braided fusion category with a twist then the last step is a change of pivotal structure on the underlying braided fusion category.

Here is a more detailed explanation of the contents of this article. In Section 2, we lay out the basic definitions and general results from the literature that we use in the sequel. Section 3.2 contains the general definition of associative zesting and the rigidity structure, the notational conventions for diagrams and the obstruction theory. Section 4 details braided zesting, in which we study the braided structures on associative zestings and the attendant obstruction theory. Section 5 studies the twist and ribbon twist structures on a braided zesting and the corresponding categorical trace and modular data. We illustrate our techniques with several examples coming from quantum groups of type $A$ in Section 6 .

\section{Preliminaries}

2.1. Group cohomology. To fix notation, we will recall the basic definitions of the standar cocycle description of group cohomology, for more details, see [26].

Let $G$ be a group and $M$ a $G$-module. We will denote by $\left(C^{n}(G, M), \delta\right)$ the cochain complex

$$
0 \rightarrow C^{0}(G, M) \rightarrow C^{1}(G, M) \rightarrow C^{2}(G, M) \rightarrow \cdots \rightarrow C^{n}(G, M) \rightarrow \cdots
$$


where $C^{0}(G, M)=M, C^{n}(G, M)$ is the abelian group of all maps from $G^{\times n}$ to $M$ and $\delta: C^{n}(G, M) \rightarrow C^{n+1}(G, M)$ is given by

$$
\begin{aligned}
\delta^{n}(f)\left(g_{1}, \cdots, g_{n+1}\right)= & g_{1} f\left(g_{2}, \cdots, g_{n+1}\right)+\sum_{i=1}^{n}(-1)^{i} f\left(g_{1}, \ldots, g_{i} g_{i+1}, \ldots, g_{n+1}\right) \\
& +(-1)^{n+1} f\left(g_{1}, \ldots, g_{n}\right) .
\end{aligned}
$$

The group cohomology of $G$ with coefficients in $M$ is defined as the cohomology of the cochain complex (2.1), that is, $H^{n}(G, M)=\operatorname{ker}\left(\delta^{n}\right) / \operatorname{Im}\left(\delta^{n-1}\right)$. As usual, we will denote by $Z^{n}(G, M)=\operatorname{ker}\left(\delta^{n}\right)$ the group of $n$-cocycles and by $B^{n}(G, M)=\operatorname{Im}\left(\delta^{n-1}\right)$ the $n$-coboundaries.

2.2. Basic definitions on fusion and modular categories. In this section, we recall some basic definitions and standard notions from [14], mainly in order to fix notation.

By a monoidal category we mean a tuple $(\mathcal{C}, \otimes, \alpha, \mathbf{1}, \lambda, \rho)$, where $\mathcal{C}$ is a category, $\otimes: \mathcal{C} \times \mathcal{C} \rightarrow \mathcal{C}$ is a bifunctor, a natural isomorphism

$$
\alpha_{X, Y, Z}:(X \otimes Y) \otimes Z \rightarrow X \otimes(Y \otimes Z),
$$

called the associator, natural isomorphisms

$$
\lambda_{X}: 1 \otimes X \rightarrow X, \quad \rho_{X}: X \otimes 1 \rightarrow X
$$

called the right and left unitors, respectively. This data must satisfy the well known pentagon and triangle axioms [14]. Hereafter we suppress the associators and unitors and denote a monoidal category by the tuple $(\mathcal{C}, \otimes, \mathbf{1})$.

A monoidal category has duals if for every $X \in \mathcal{C}$ there is an object $X^{*} \in \mathcal{C}$ and morphism $\epsilon_{X}: X^{*} \otimes X \rightarrow \mathbf{1}, \delta_{X}: \mathbf{1} \rightarrow X \otimes X^{*}$ satisfying the zig-zag axioms:

$$
\begin{gathered}
\left(\operatorname{id}_{X} \otimes \epsilon_{X}\right) \circ \alpha_{X, X^{*}, X} \circ\left(\delta_{X} \otimes \operatorname{id}_{X}\right)=\operatorname{id}_{X} \\
\left(\epsilon_{X} \otimes \operatorname{id}_{X^{*}}\right) \circ \alpha_{X^{*}, X, X^{*}}^{-1} \circ\left(\operatorname{id}_{X^{*}} \otimes \delta_{X}\right)=\operatorname{id}_{X^{*}} .
\end{gathered}
$$

A monoidal category with duals is called rigid if for every $X \in \mathcal{C}$ there is ${ }^{*} X \in \mathcal{C}$ such that $\left({ }^{*} X\right)^{*} \cong X$.

We will denote by $\mathbb{k}$ an algebraically closed field of characteristic zero. By a fusion category, we mean a semisimple $\mathbb{k}$-linear abelian rigid monoidal category $(\mathcal{C}, \otimes, \mathbf{1})$ such that the unit object $\mathbf{1}$ of $\mathcal{C}$ is simple and there are finite many isomorphism classes of simple objects. The set of isomorphism classes of simple objects of $\mathcal{C}$ is denoted by $\operatorname{Irr}(\mathcal{C})$. By a fusion subcategory of a fusion category, we mean a full monoidal abelian subcategory.

For a fusion category $\mathcal{C}$, we will denote by $\mathcal{C}_{\mathrm{pt}}$ the full fusion subcategory generated by $\otimes$-invertible objects. We will denote by $\operatorname{Inv}(\mathcal{C})$ the group of isomorphism classes of $\otimes$-invertible objects of $\mathcal{C}$ under the tensor product. A fusion category is called pointed if every simple object is $\otimes$-invertible. 
Example 2.1 (Pointed fusion categories). Let $G$ be a finite group. A (normalized) 3-cocycle $\omega \in Z^{3}\left(G, \mathbb{k}^{\times}\right)$is a map $\omega: G \times G \times G \rightarrow \mathbb{k}^{\times}$such that

$$
\omega(a b, c, d) \omega(a, b, c d)=\omega(a, b, c) \omega(a, b c, d) \omega(b, c, d), \quad \omega(a, 1, b)=1,
$$

for all $a, b, c, d \in G$.

Let us recall the description of the pointed fusion category $\operatorname{Vec}_{G}^{\omega}$. The objects of $\operatorname{Vec}_{G}^{\omega}$ are $G$-graded finite dimensional vector spaces $V=\bigoplus_{g \in G} V_{g}$. Morphisms are $G$ linear $G$-homogeneous maps. The tensor product of $V=\oplus_{g \in G} V_{g}$ and $W=\oplus_{g \in G} W_{g}$ is $V \otimes W$ as vector space, with $G$-grading

$$
(V \otimes W)_{g}=\bigoplus_{h \in G} V_{h} \otimes W_{h^{-1} g} .
$$

For objects $V, W, Z \in \operatorname{Vec}_{G}^{\omega}$ the associativity constraint is defined by

$$
\begin{aligned}
a_{V, W, Z}:(V \otimes W) \otimes Z & \rightarrow V \otimes(W \otimes Z) \\
\left(v_{g} \otimes w_{h}\right) \otimes z_{k} & \mapsto \omega(g, h, k) v_{g} \otimes\left(w_{h} \otimes z_{k}\right)
\end{aligned}
$$

for all $g, h, k \in G, v_{g} \in V_{g}, w_{h} \in W_{h}, z_{k} \in Z_{k}$. The unit objects is $\mathbb{k}_{e}$, the vector space $\mathbb{k}$ graded only by the identity element $e \in G$.

For $V \in \operatorname{Vec}_{G}^{\omega}$, the dual object is $V^{*}=\operatorname{Hom}_{\mathbb{k}}(V, \mathbb{k})$, with $G$-grading $V_{g}^{*}=\operatorname{Hom}_{\mathbb{k}}\left(V_{g^{-1}}, \mathbb{k}\right)$ and

$$
\begin{aligned}
\epsilon_{V}: V^{*} \otimes V & \rightarrow \mathbb{k}_{e}, & \delta_{V}: \mathbb{k}_{e} & \rightarrow V \otimes V^{*} \\
\alpha_{h} \otimes v_{g} & \mapsto \omega\left(g, g^{-1}, g\right)^{-1} \alpha_{h}\left(v_{g}\right), & 1 & \mapsto \sum v_{i} \otimes v^{i}
\end{aligned}
$$

where $g, h \in G, v_{g} \in V_{g}^{*}$ and $\alpha_{h} \in V_{h}^{*}$, and $\delta_{V}$ is the usual coevaluation map of finite dimensional vector spaces.

2.3. Pivotal and spherical fusion categories. If $\mathcal{C}$ is a monoidal category with duals, we can define a monoidal functor $(-)^{*}: \mathcal{C} \rightarrow \mathcal{C}^{o p}$, where $\mathcal{C}^{o p}$ is the opposite category with tensor product $X \otimes^{o p} Y:=Y \otimes X$. Here, for a morphism $f: X \rightarrow Y$, we have that $f^{*}: Y^{*} \rightarrow X^{*}$ is given by $\left(\epsilon_{Y} \otimes \mathrm{id}_{X^{*}}\right) \circ\left(\mathrm{id}_{Y^{*}} \otimes f \otimes \mathrm{id}_{X^{*}}\right) \circ\left(\operatorname{id}_{Y^{*}} \otimes \delta_{X}\right)$.

A pivotal structure on a rigid monoidal category is monoidal natural isomorphism $\psi: \operatorname{Id}_{\mathcal{C}} \rightarrow(-)^{* *}$. The left and right pivotal traces of an endomorphism $f: X \rightarrow X$ are given by

$$
\begin{aligned}
& \operatorname{Tr}_{L}(f)=\epsilon_{X} \circ\left(\operatorname{id}_{X^{*}} \otimes f\right) \circ\left(\operatorname{id}_{X^{*}} \otimes \psi_{X}^{-1}\right) \circ \delta_{X^{*}} \\
& \operatorname{Tr}_{R}(f)=\epsilon_{X^{*}} \circ\left(\psi_{X} \otimes \operatorname{id}_{X^{*}}\right) \circ\left(f \otimes \operatorname{id}_{X^{*}}\right) \circ \delta_{X} .
\end{aligned}
$$

A rigid monoidal category with a pivotal structure is called pivotal monoidal category.

A spherical monoidal category is a pivotal category such that the left and right traces of every endomorphism coincide. For spherical categories the left and right trace of an endomorphism $f$ will be denoted simply by $\operatorname{Tr}(f)$. The quantum dimension or just the dimension of an object $X \in \mathcal{C}$ is $\operatorname{dim}(X)=\operatorname{Tr}\left(\operatorname{id}_{X}\right)$. 
Example 2.2. The pointed fusion category $\operatorname{Vec}_{G}^{\omega}$ has a canonical pivotal structure given by

$$
\psi_{V}=\bigoplus_{g \in G} \omega\left(g^{-1}, g, g^{-1}\right) \mathrm{id}_{V_{g}} .
$$

Any other pivotal structure differs from the canonical one by a linear character $\chi$ : $G \rightarrow \mathbb{k}^{\times}$, given by

$$
\psi_{V}^{\chi}=\bigoplus_{g \in G} \chi(g) \omega\left(g^{-1}, g, g^{-1}\right) \operatorname{id}_{V_{g}} .
$$

The pivotal structure $\psi_{V}^{\chi}$ is spherical if and only if $\chi(g) \in\{1,-1\}$.

2.4. Premodular and modular tensor categories. A braiding for a monoidal category $\mathcal{B}$ is a natural isomorphism

$$
c_{X, Y}: X \otimes Y \rightarrow Y \otimes X, \quad X, Y \in \mathcal{B}
$$

satisfying the two well known hexagon axioms. A braided fusion category is a fusion category with a braiding. A braided category is called symmetric if $c_{X, Y}^{-1}=c_{Y, X}$ for all $X, Y \in \mathcal{B}$. The centralizer of a set of objects $\mathcal{S}$ is the subcategory with objects

$$
C_{\mathcal{S}}(\mathcal{B}):=\left\{X \in \mathcal{B}: c_{Y, X} \circ c_{X, Y}=\operatorname{id}_{X \otimes Y}, X \in \mathcal{S}\right\}
$$

An object $Y$ is called transparent if $C_{\{Y\}}(\mathcal{B})=\mathcal{B}$, so that every object is transparent in a symmetric category.

In [9], Deligne establishes that every symmetric fusion category is braided equivalent to one of the following:

- Tannakian categories. These take the form $\operatorname{Rep}(G)$ of finite dimensional representations of a finite group $G$, with the standard braiding $c_{X, Y}(x \otimes y):=y \otimes x$.

- Super-Tannakian categories. These are categories of finite-dimensional representations of finite super-groups, denoted by $\operatorname{Rep}(G, z)$. A finite super-group is a pair $(G, z)$, where $G$ is a finite group and $z$ is a central element of order two. As fusion categories they can be understood as $\operatorname{Rep}(G)$ but with a nonstandard braiding $c^{z}$ :

An irreducible representation of $G$ is called odd if $z$ acts as the scalar -1 , and even if $z$ acts as the identity. If the degree of a simple object $X$ is denoted by $|X| \in\{0,1\}$, then a braiding on $\operatorname{Rep}(G)$ is given by $c_{X, Y}^{z}(x \otimes y):=(-1)^{|x||y|} y \otimes x$ for $x \in X$ and $y \in Y$, where $X$ and $Y$ are simple representations.

A twist for a braided category is a natural isomorphism of the identity

$$
\theta_{X}: X \rightarrow X
$$

such that

$$
\theta_{X \otimes Y}=c_{Y, X} \circ c_{X, Y} \circ\left(\theta_{X} \otimes \theta_{Y}\right),
$$

for all $X, Y \in \mathcal{C}$. It is well-known (see e.g. [7]) that pivotal structures on a braided fusion category are in bijective correspondence with twists. A twist on a braided fusion category is called a ribbon twist if $\theta_{X^{*}}=\theta_{X}^{*}$ for all $X \in \mathcal{C}$, and ribbon twists correspond to spherical pivotal structures under the pivotal/twist correspondence. We will recall this correspondence briefly for more details, see [19, Appendix A.2]. 
Let $\mathcal{C}$ be a rigid braided monoidal category. The Drinfeld isomorphism is a natural isomorphism $u: \operatorname{Id} \rightarrow(-)^{* *}$ given by

$$
u_{X}:=\left(\epsilon_{X} \otimes \operatorname{id}_{X^{* *}}\right) \circ\left(\operatorname{id}_{X^{*}} \otimes c_{X^{* *}, X}^{-1}\right) \circ\left(\delta_{X^{*}} \otimes \operatorname{id}_{X}\right): X \rightarrow X^{* *} .
$$

Given a twist $\theta$, the natural isomorphism

$$
\psi_{X}=u_{X} \circ \theta_{X}, \quad X \in \mathcal{C}
$$

is a pivotal structure. Conversely, if $\psi_{X}: X \rightarrow X^{* *}$ is a pivotal structure then

$$
\theta_{X}=u_{X}^{-1} \circ \psi_{X}, \quad X \in \mathcal{C},
$$

is twist.

A braided fusion category with a spherical pivotal structure (or equivalently a ribbon twist) is called a premodular tensor category.

Following [1], we define the modular data of a premodular category as the following pair of matrices with respect to the a basis given by a fixed ordering of $\operatorname{Irr}(\mathcal{B})$ :

(i) $S$-matrix. $S_{X, Y}=\operatorname{Tr}\left(c_{Y^{*}, X} \circ c_{X, Y^{*}}\right)$,

(ii) T-matrix. $T_{X, Y}=\theta_{X} \delta_{X, Y}$.

Notice that the categorical dimension of a simple object $X$ is $\operatorname{dim}(X)=S_{X, \mathbf{1}}$. A premodular tensor category is called modular if $S$ is invertible. Any modular tensor category defines a projective representation of the modular group $\mathrm{SL}(2, \mathbb{Z})$ as follows: the matrices

$$
\mathfrak{s}:=\left(\begin{array}{cc}
0 & -1 \\
1 & 0
\end{array}\right), \quad \quad \mathfrak{t}:=\left(\begin{array}{ll}
1 & 1 \\
0 & 1
\end{array}\right)
$$

generates $\mathrm{SL}(2, \mathbb{Z})$ and by [1] the assignment

$$
\mathfrak{s} \mapsto \frac{1}{\sqrt{\operatorname{dim}(\mathcal{B})}} S, \quad \mathfrak{t} \mapsto T
$$

defines a projective representation, where $\operatorname{dim}(\mathcal{B})=\sum_{X \in \operatorname{Irr}(\mathcal{B})} \operatorname{dim}(X)^{2}$.

Remark 2.3. In [14 the $S$-matrix and $T$-matrix are defined by

$$
S_{X, Y}^{\prime}=\operatorname{Tr}\left(c_{Y, X} \circ c_{X, Y}\right), \quad T_{X, Y}^{\prime}=\theta_{X}^{-1} \delta_{X, Y} .
$$

the $\left(S^{\prime}, T^{\prime}\right)$ and $(S, T)$ are directly related by $T^{\prime}=T^{-1}$ and $S^{\prime}=S^{-1}$, see [14, Proposition 8.14.2].

Example 2.4 (Pointed braided fusion categories). Let $\operatorname{Vec}_{G}^{\omega}$ be a pointed fusion category, with $G$ abelian. A braiding on $\operatorname{Vec}_{G}^{\omega}$ is defined by a function $c: G \times G \rightarrow \mathbb{K}^{\times}$ as

$$
\begin{aligned}
c_{V, W}: V \otimes W & \rightarrow W \otimes V \\
v_{g} \otimes w_{h} & \mapsto c(g, h) w_{h} \otimes v_{g} .
\end{aligned}
$$


The function $c$ must satisfy the following equations:

$$
\begin{aligned}
& \frac{c(g, h k)}{c(g, h) c(g, k)}=\frac{\omega(g, h, k) \omega(h, k, g)}{\omega(h, g, k)} \\
& \frac{c(g h, k)}{c(g, k) c(h, k)}=\frac{\omega(g, k, h)}{\omega(g, h, k) \omega(k, g, h)},
\end{aligned} \quad \text { for all } g, h, k \in G,
$$

These equations correspond to the hexagon axioms. A pair $(\omega, c)$ satisfying (2.3) is called an abelian 3-cocycle. Following [12, 13] we denote by $Z_{a b}^{3}\left(G, \mathbb{k}^{\times}\right)$the abelian group of all abelian 3-cocycles $(\omega, c)$.

An abelian 3-cocycle $(\omega, c) \in Z_{a b}^{3}\left(G, \mathbb{k}^{\times}\right)$is called an abelian 3-coboundary if there is $\alpha: G^{\times 2} \rightarrow \mathbb{k}^{\times}$, such that

$$
\begin{aligned}
\omega(g, h, k) & =\frac{\alpha(g, h) \alpha(g h, k)}{\alpha(g, h k) \alpha(h, k)} \\
c(g, h) & =\frac{\alpha(g, h)}{\alpha(h, g)},
\end{aligned}
$$

for all $g, h, k \in G$.

$B_{a b}^{3}\left(G, \mathbb{k}^{\times}\right)$denotes the subgroup of $Z_{a b}^{3}\left(G, \mathbb{k}^{\times}\right)$of abelian 3-coboundaries. The quotient group $H_{a b}^{3}\left(G, \mathbb{k}^{\times}\right):=Z_{a b}^{3}\left(G, \mathbb{k}^{\times}\right) / B_{a b}^{3}\left(G, \mathbb{k}^{\times}\right)$is called the third group of abelian cohomology of $G$.

Under the correspondence between pivotal structures and twist, we have that pivotal structure $\psi^{\chi}$ in (2.2) corresponds to the twist

$$
\theta_{V}^{\chi}=\bigoplus_{g \in G} \chi(g) c(g, g) \operatorname{id}_{V_{g}} .
$$

2.5. Graded fusion categories and tensor natural isomorphisms of the identity functor. Let $G$ be a finite group. A fusion category $\mathcal{C}$ is $G$-graded if there is a decomposition

$$
\mathcal{C}=\bigoplus_{g \in G} \mathcal{C}_{g}
$$

of $\mathcal{C}$ into a direct sum of full abelian subcategories such that the tensor product of $\mathcal{C}$ maps $\mathcal{C}_{g} \times \mathcal{C}_{h}$ to $\mathcal{C}_{g h}$ for all $g, h \in G$. We will say that the $G$-grading is faithful if $\mathcal{C}_{g} \neq 0$ for all $g \in G$.

Example 2.5. Let $\mathcal{C}$ be a fusion category and $A=\widehat{\operatorname{Aut}_{\otimes}\left(\operatorname{Id}_{\mathcal{C}}\right)}$ the group of linear characters of $\mathrm{Aut}_{\otimes}\left(\mathrm{Id}_{\mathcal{C}}\right)$, the abelian group of tensor automorphism of the identity functor. Then $\mathcal{C}=\bigoplus_{\gamma \in A} \mathcal{C}_{\gamma}$ is faithfully $A$-graded, where

$$
\mathcal{C}_{\gamma}=\left\{X \in \mathcal{C}: \rho_{X}=\gamma(\rho) \operatorname{id}_{X}, \quad \forall \rho \in \operatorname{Aut}_{\otimes}\left(\operatorname{Id}_{\mathcal{C}}\right)\right\}
$$

It was proved in [18, Theorem 3.5] that any fusion category $\mathcal{C}$ is naturally graded by a group $U(\mathcal{C})$, called the universal grading group of $\mathcal{C}$, and the adjoint fusion subcategory $\mathcal{C}_{a d}$ (tensor generated by all subobjects of $X \otimes X^{*}$ for all simple objects $X)$ is the trivial component of this grading. Additionally, any other faithful grading arises from a quotient of $U(\mathcal{C})$. 
Definition 2.6. Let $\mathcal{C}$ be a faithfully $G$-graded fusion category. We will denote by $\operatorname{Aut}_{\otimes}^{G}\left(\operatorname{Id}_{\mathcal{C}}\right)$ the abelian group of all tensor natural isomorphisms of the identity $\Phi \in$ Aut $_{\otimes}\left(\operatorname{Id}_{\mathcal{C}}\right)$ such that $\Phi_{X}=\operatorname{id}_{X}$ for all $X \in \mathcal{C}_{e}$.

Let $\mathcal{C}$ be a faithfully $G$-graded fusion category. It follows as in [18, Proposition 3.9] that given $\gamma \in \widehat{G}$ the assignment $\Phi_{\gamma} \in \operatorname{Aut}_{\otimes}\left(\operatorname{Id}_{\mathcal{C}}\right)$ given by

$$
\Phi_{\gamma}\left(X_{s}\right)=\gamma(s) \operatorname{id}_{X_{s}}, \quad X_{s} \in \mathcal{C}_{s}
$$

defines a group homorphism from $\widehat{G}$ to $\operatorname{Aut}_{\otimes}^{G}\left(\operatorname{Id}_{\mathcal{C}}\right)$.

The following result is a direct consequence of [18, Proposition 3.9].

Proposition 2.7. Let $\mathcal{C}$ be a faithfully $G$-graded fusion category. The group homomorhism $\Phi: \widehat{G} \rightarrow \operatorname{Aut}_{\otimes}^{G}\left(\operatorname{Id}_{\mathcal{C}}\right)$ is an isomorphism. In particular $\left.\Phi: \widehat{U(\mathcal{C}}\right) \cong \operatorname{Aut}_{\otimes}\left(\operatorname{Id}_{\mathcal{C}}\right)$ so that any $\psi \in \mathrm{Aut}_{\otimes}\left(\mathrm{Id}_{\mathcal{C}}\right)$ is constant on $U(\mathcal{C})$-graded components.

Let $\mathcal{B}$ be a braided monoidal category and $a \in \mathcal{B}$ an invertible object. Then we can define a natural isomorphism of the identity functor $\chi_{a} \in \operatorname{Aut}\left(\operatorname{Id}_{\mathcal{B}}\right)$ by the equality $\chi_{a}(X) \otimes \mathrm{id}_{a}=c_{a, X} \circ c_{X, a}$ for $X \in \operatorname{Obj}(\mathcal{B})$, see Figure 1.

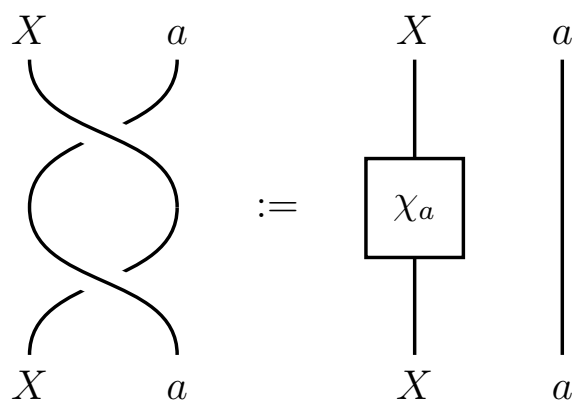

Figure 1. Definition of $\chi$

The following result is essentially the same as [14, Lemma 8.22.9]. Note that the result does not use semisimplicity or finiteness.

Proposition 2.8. Let $\mathcal{B}$ be a braided tensor category and $a \in \mathcal{B}$ an invertible object. Then

(i) $\chi_{a}$ is a monoidal natural isomorphism.

(ii) The map $\chi: \operatorname{Inv}(\mathcal{B}) \rightarrow \operatorname{Aut}_{\otimes}\left(\operatorname{Id}_{\mathcal{B}}\right)$ is a group morphism.

(iii) The kernel of $\chi$ is the group of transparent invertible objects.

Corollary 2.9. Let $\mathcal{B}$ be a braided fusion category. Then $\mathcal{B}$ is faithfully graded over the group $\operatorname{Inv}(\widehat{\mathcal{B}) / \operatorname{ker}}(\chi)$ (linear characters over $\operatorname{Inv}(\mathcal{B}) / \operatorname{ker}(\chi)$ ) as follows:

$$
\mathcal{B}_{\gamma}=\left\{X \in \mathcal{B}: \chi_{a}(X)=\gamma(a) \operatorname{id}_{X}, \quad \forall a \in \operatorname{Inv}(\mathcal{B})\right\}, \quad \gamma \in \operatorname{Inv}(\widehat{\mathcal{B}) / \operatorname{ker}}(\chi) .
$$

Proof. Since the induced map $\chi: \operatorname{Inv}(\mathcal{B}) / \operatorname{ker}(\chi) \rightarrow \operatorname{Aut}_{\otimes}\left(\operatorname{Id}_{\mathcal{B}}\right)$ is injective, then the induced surjective map $\left.\chi^{*}:{\widehat{\operatorname{At}} \otimes\left(\operatorname{Id}_{\mathcal{B}}\right.}\right) \rightarrow \operatorname{Inv}(\widehat{\mathcal{B}) / \operatorname{ker}}(\chi)$ defines a faithful $\operatorname{Inv}(\widehat{\mathcal{B}) / \operatorname{ker}}(\chi)$ grading by Example 2.5 . 
2.6. Conventions and Graphical calculus. In the following sections $\mathcal{C}$ is a fusion category, which we may assume is strict without loss of generality by MacLane's Strictness and Coherence Theorems. In particular we can ignore associators and draw diagrams modulo isotopy that preserves the order of objects, for example

$$
||=|+|=\mid
$$

Our diagrams are oriented top to bottom and left to right. Our convention for braiding diagrams is that for positive braids the $i+1$ st strand passes over the $i$ th strand.

\section{Associative Zestings}

3.1. Relative centralizer of monoidal subcategories. Let $\mathcal{C}$ be a fusion category and $\mathcal{D} \subset \mathcal{C}$ a fusion subcategory. The relative centralizer $R_{\mathcal{D}}(\mathcal{C})$ is the fusion subcategory of the Drinfeld center $\mathcal{Z}(\mathcal{C})$ whose objects are pairs $\left(X, \sigma_{X,-}\right)$ where $X \in \mathcal{D}$ and

$$
\sigma_{X,-}=\left\{\sigma_{X, V}: X \otimes V \rightarrow V \otimes X\right\}_{V \in \mathcal{C}}
$$

is a family of isomorphisms natural in $V \in \mathcal{C}$ such that

$$
\sigma_{X, V \otimes W}=\left(\operatorname{id}_{V} \otimes \sigma_{X, W}\right) \circ\left(\sigma_{X, V} \otimes \operatorname{id}_{W}\right)
$$

for all $V, W \in \mathcal{C}$.

While $\mathcal{C}$ is not a priori braided, since $R_{\mathcal{D}}(\mathcal{C})$ is a subcategory of the braided Drinfeld center $\mathcal{Z}(\mathcal{C})$ we can use crossings in our graphical calculus when objects of the relative center are involved. For example, Equation 3.1 becomes

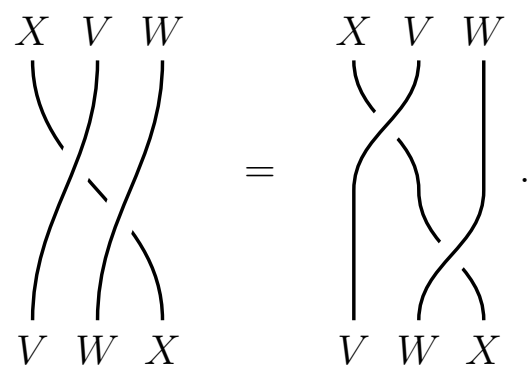

A morphism $f:\left(X, \sigma_{X,-}\right) \rightarrow\left(Y, \sigma_{Y,-}\right)$ in $R_{\mathcal{D}}(\mathcal{C})$ is a morphism $f: X \rightarrow Y$ in $\mathcal{D}$ such that $\left(\operatorname{id}_{V} \otimes f\right) \circ \sigma_{X, V}=\sigma_{Y, V} \circ\left(f \otimes \operatorname{id}_{V}\right)$ for all $V \in \mathcal{C}$. In pictures, 


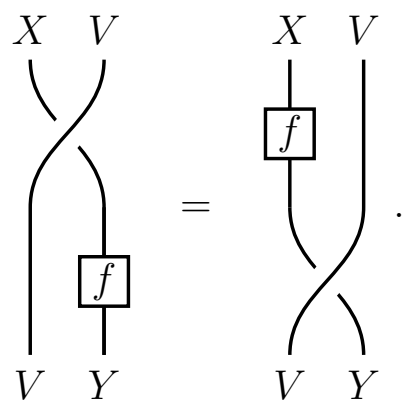

The isomorphism $\sigma$ will be called the relative half braiding. The category $R_{\mathcal{D}}(\mathcal{C})$ is monoidal with tensor product given by

$$
\left(X, \sigma_{X,-}\right) \otimes\left(Y, \sigma_{Y,-}\right)=\left(X \otimes Y,\left(\sigma_{X,-} \otimes \mathrm{id}\right) \circ\left(\mathrm{id} \otimes \sigma_{Y,-}\right)\right),
$$

and unit object $(\mathbf{1}, \mathrm{id})$.

Remark 3.1. The notion of relative center was defined in [17]. This concept is closely related to the one of relative centralizer introduced above. If $\mathcal{C}$ is a fusion category and $\mathcal{D} \subset \mathcal{C}$ a fusion subcategory, then the relative centralizer $R_{\mathcal{D}}(\mathcal{C})$ is a full fusion subcategory of the relative center $\mathcal{Z}_{\mathcal{D}}(\mathcal{C})$ (see [17, Definition 2.1] for the precise definition). The relative centralizer $R_{\mathcal{D}}(\mathcal{C})$ is also a full fusion subcategory of the Drinfeld centers $\mathcal{Z}(\mathcal{D})$ and $\mathcal{Z}(\mathcal{C})$. In particular, $R_{\mathcal{D}}(\mathcal{C})$ is braided. In the case that $\mathcal{C}=\mathcal{D}$, the fusion category $R_{\mathcal{C}}(\mathcal{C})$ coincides with the Drinfeld center $\mathcal{Z}(\mathcal{C})$.

\subsection{Associative zesting.}

Definition 3.2. Let $G$ be a group and $\mathcal{C}=\oplus_{g \in G} \mathcal{C}_{g}$ be a faithfully $G$-graded fusion category.

An associative $G$-zesting $\lambda$ for $\mathcal{C}$ consists of the following data:

(1) A map

$$
\lambda: G \times G \rightarrow\left[R_{\mathcal{C}_{e}}(\mathcal{C})\right]_{p t}, \quad(g, h) \mapsto \lambda(g, h)
$$

(2) For each $\left(g_{1}, g_{2}, g_{2}\right) \in G^{\times 3}$ an isomorphism

$$
\lambda_{g_{1}, g_{2}, g_{3}}: \lambda\left(g_{1}, g_{2}\right) \otimes \lambda\left(g_{1} g_{2}, g_{3}\right) \rightarrow \lambda\left(g_{2}, g_{3}\right) \otimes \lambda\left(g_{1}, g_{2} g_{3}\right)
$$

which we represent graphically by

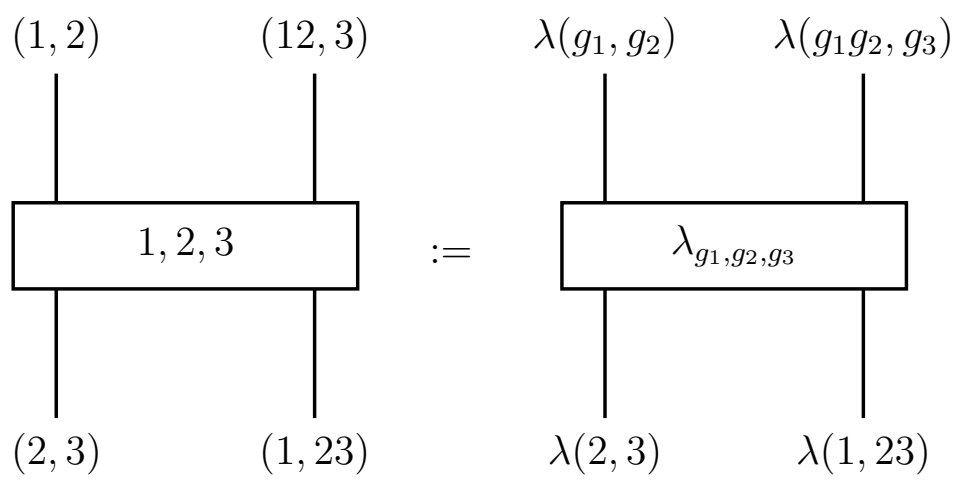


such that for any $\left(g_{1}, g_{2}, g_{3}, g_{4}\right) \in G^{\times 4}$ the equation in Figure 2 holds (see Remark 3.3(1) for notation conventions).

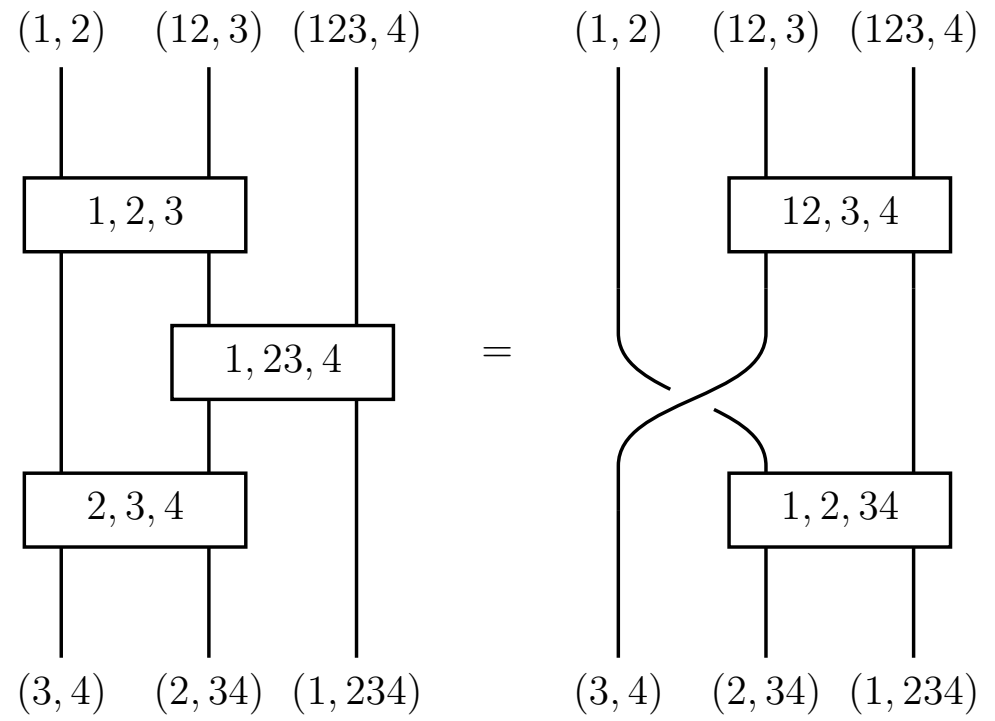

Figure 2. Associative zesting constraint

Moreover, we impose the following normalization conditions:

$$
\begin{aligned}
\lambda\left(e, g_{1}\right) & =\lambda\left(g_{1}, e\right)=\mathbf{1}, \\
\lambda\left(g_{1}, e, g_{2}\right) & =\operatorname{id}_{\lambda\left(g_{1}, g_{2}\right)} .
\end{aligned}
$$

Remark 3.3. (1) The label 1,2,3 of the box on the right-hand side of Definition $3.2(2)$ has enough information to recover the target and source of the isomorphism $\lambda_{g_{1}, g_{2}, g_{3}}$, so we suppress the labels on the strands. For further notational convenience we identify $g_{i}$ with the index $i$ so that, for example $g_{1} g_{2}$ becomes 12 in Figure 2 and in subsequent figures.

(2) Condition (2) of Definition 3.2 implies that $\lambda$ in (1) is a 2-cocycle in the sense that $\left(g_{1}, g_{2}\right) \mapsto\left[\lambda\left(g_{1}, g_{2}\right)\right]$ satisfies the 2-cocycle condition, where we interpret $\left[\lambda\left(g_{1}, g_{2}\right)\right]$ as an element of the group of invertible objects.

(3) It follows from the assumptions (3.2), (3.3), and the associative zesting condition that we also have $\lambda\left(e, g_{1}, g_{2}\right)=c_{1, \lambda\left(g_{1}, g_{2}\right)}=\operatorname{id}_{\lambda\left(g_{1}, g_{2}\right)}$, and $\lambda\left(g_{1}, e, g_{2}\right)=$ $c_{\lambda\left(g_{1}, g_{2}\right), \mathbf{1}}=\mathrm{id}_{\lambda\left(g_{1}, g_{2}\right)}$.

Proposition 3.4. Let $G$ be a finite group and $\mathcal{C}$ a faithfully $G$-graded fusion category. Given an associative $G$-zesting $\lambda$, we can define a new faithfully G-graded fusion category $\mathcal{C}^{\lambda}:=\left(\mathcal{C}, \stackrel{\lambda}{\otimes}, \boldsymbol{a}^{\lambda}\right)$, where the tensor product $\stackrel{\lambda}{\otimes}$ is defined as

$$
V_{g_{1}} \stackrel{\lambda}{\otimes} W_{g_{2}}:=V_{g_{1}} \otimes W_{g_{2}} \otimes \lambda\left(g_{1}, g_{2}\right),
$$


BRAIDED ZESTING

the associativity constraint $\boldsymbol{a}_{V_{g_{1}}, W_{g_{2}}, Z_{g_{3}}}^{\lambda}$ by
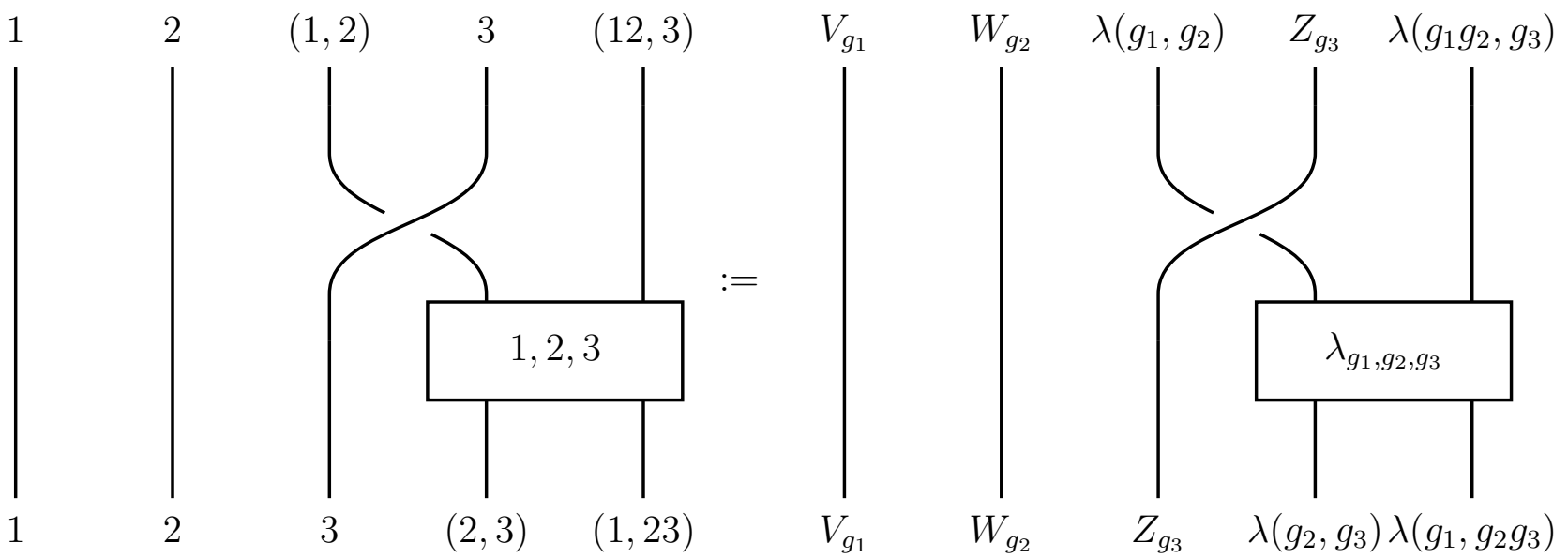

and the same unit object and unit constraint as $\mathcal{C}$.

Proof. An associative zesting is a particular case of the construction of a faithfully graded fusion category given in [15, Section 8]. For the convenience of the reader, we will check the pentagon axiom. The pentagon axiom is equivalent to the equality in Figure 3 .

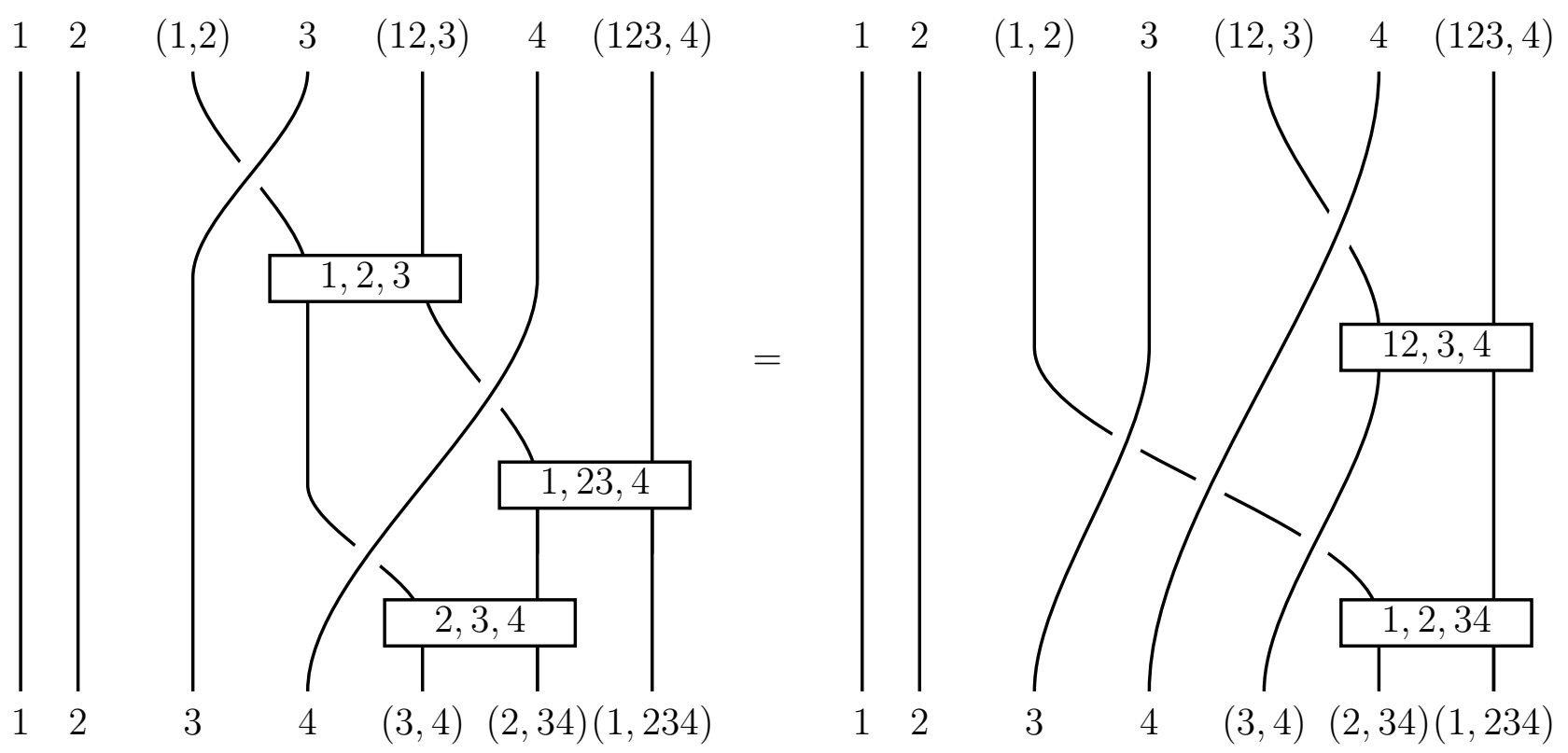

Figure 3. Pentagon axiom that must be satisfied by the zested associators.

Using the graphical calculus is easy to check that the equality depicted in Figure 3 is equivalent to the one in Figure 4. Now, the associative zesting condition of Figure 
2 implies the equality in Figure 4 and therefore the pentagon axiom in the zested category.

Finally, the fact that $\mathbf{1}$ is the unit object with the same unit constraints follows directly from the definition of the tensor product of $\mathcal{C}^{\lambda}$ and the conditions (3.2) and (3.3).

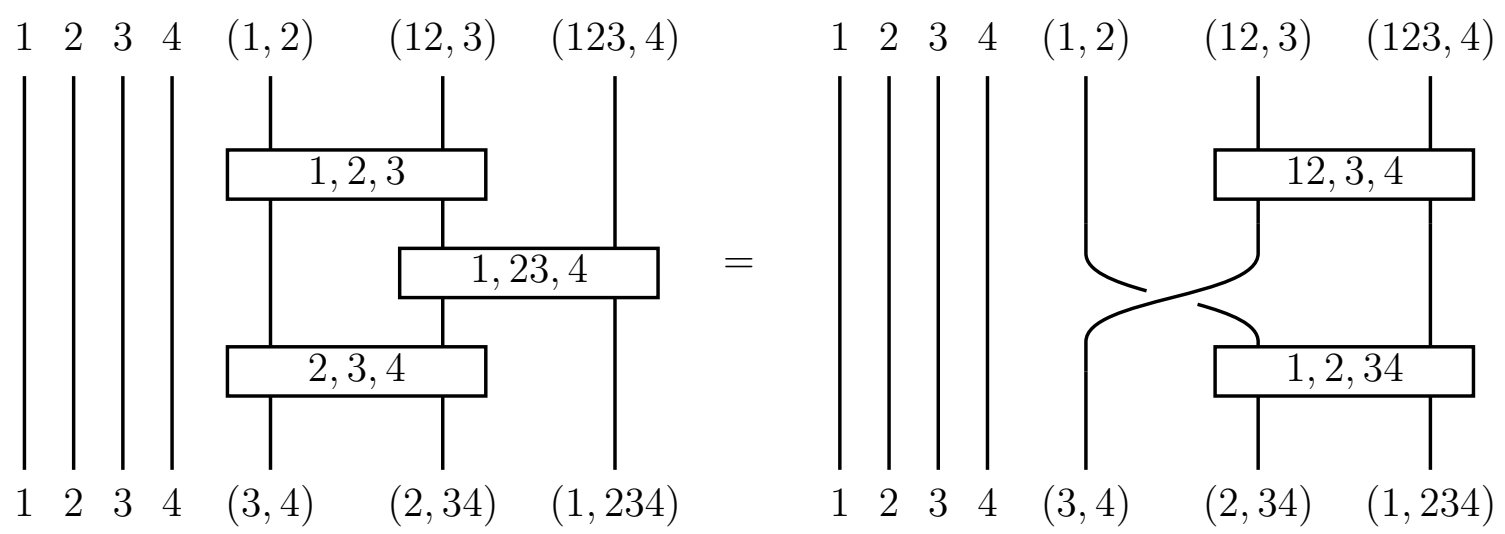

FiguRE 4. Equivalent formulation of the pentagon axiom from Figure 3.

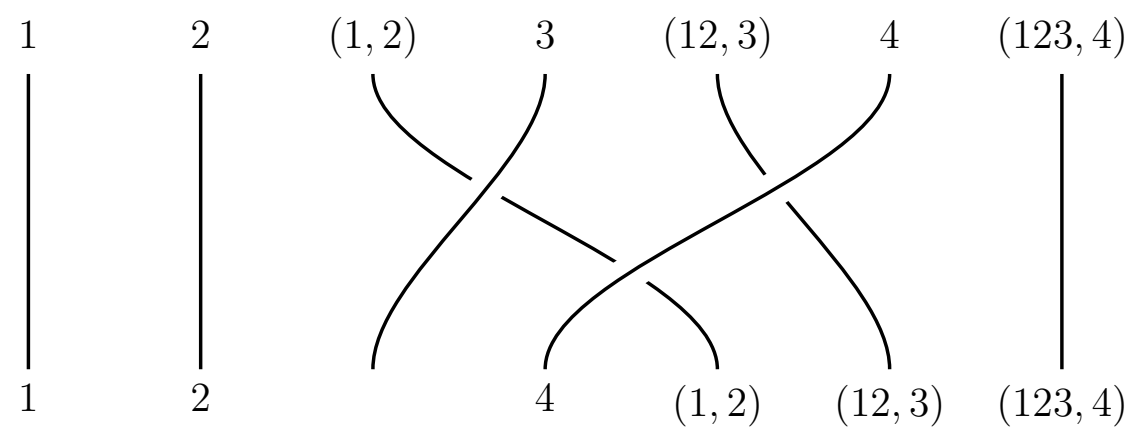

Remark 3.5.

Given a map $\lambda: G \times G \rightarrow\left[R_{\mathcal{C}_{e}}(\mathcal{C})\right]_{p t}$ there are at least three associated bifunctors, namely

$$
\begin{aligned}
& V_{g} \otimes{ }^{1} W_{h}:=V_{g} \otimes W_{h} \otimes \lambda(g, h), \\
& V_{g} \otimes^{2} W_{h}:=V_{g} \otimes \lambda(g, h) \otimes W_{h} \\
& V_{g} \otimes{ }^{3} W_{h}:=\lambda(g, h) \otimes V_{g} \otimes W_{h} .
\end{aligned}
$$

They are easily seen to be naturally isomorphic (proof supplied upon request), so our choice of $\otimes^{1}$ is no loss of generality. 
Example 3.6. As a special case of our construction we can recover some examples found in [21] from the modular $\mathbb{Z} / N$-graded category $S U(N)_{k}$. In loc. cit. they classify fusion categories with the same fusion rules as $S U(N)_{k}$, showing that any such category is obtained from $S U(N)_{k}$ by either changing the quantum parameter $q$ or twisting the associativity morphisms by a 3-cocycle, or both. If we choose the trivial 2-cocycle $\lambda: \mathbb{Z} / N \times \mathbb{Z} / N \rightarrow \mathcal{C}_{p t} \cap \mathcal{C}_{0}$, i.e., $\lambda(a, b)=\mathbf{1}$ then the second associative zesting constraint (Figure 2) is simply the condition that $\lambda_{a, b, c}$ is a (normalized) 3cocycle on $\mathbb{Z} / N$. Thus the associative zestings of $S U(N)_{k}$ with trivial 2-cocycle are precisely the ones obtained in [21] by twisting the associativity morphisms. We will study some cases with non-trivial 2-cocycle below.

\subsection{Rigidity of associative zesting.}

Lemma 3.7. Let $\mathcal{C}$ be a fusion category and $X, Y \in \mathcal{C}$ simple objects such that $\operatorname{Hom}(1, X \otimes Y) \neq 0$. If

$$
\phi: Y \otimes X \rightarrow 1, \quad \rho: 1 \rightarrow X \otimes Y
$$

is a pair of non-zero morphisms, then the scalar $z(\phi, \rho) \in \mathbb{k}$ defined by

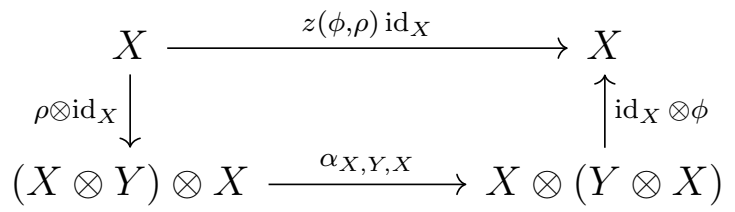

is non-zero and the triple $(Y, \phi, z(\phi, \rho) \rho)$ is a dual of $X$. Moreover, given a non-zero map $\phi: Y \otimes X \rightarrow 1$ the map $z(\phi, \rho) \rho$ does not depend in the choice of $\rho$, that is, if $\rho, \rho^{\prime}: \mathbf{1} \rightarrow X \otimes Y$ are non-zero maps, then $z(\phi, \rho) \rho=z\left(\phi, \rho^{\prime}\right) \rho^{\prime}$.

Proof. In a fusion category we have that, for simple objects $X$ and $Y, \operatorname{Hom}(\mathbf{1}, X \otimes$ $Y) \neq 0$ if and only if $Y \cong X^{*}$. Moreover, since in that case, $\operatorname{Hom}(\mathbf{1}, X \otimes Y)$ is one dimensional, there are non-zero scalars $c_{1}, c_{2} \in \mathbb{k}^{\times}$such that $\epsilon_{X}=c_{1} \phi$ and $\delta_{X}=c_{2} \rho$ where $\left(\delta_{X}, \epsilon_{X}\right)$ defines a dual for $X$. Clearly $z(\phi, \rho)=c_{1} c_{2}$, thus $z(\phi, \rho)$ is non-zero. Moreover, we have that $(\phi, z(\phi, \rho) \rho)=\left(c_{1}^{-1} \epsilon_{X}, c_{1} \delta_{X}\right)$. Hence $(\phi, z(\phi, \rho) \rho)$ also defines a dual for $X$.

For the uniqueness, note that $z(\phi, \rho) \rho=c_{1} c_{2} \rho=c_{1} \delta_{X}$. Since $c_{1}$ only depends on $\phi$ then $z(\phi, \rho) \rho$ only depends on $\phi$.

Let $\mathcal{C}$ be a faithfully $G$-graded fusion category and $\lambda$ an associative zesting. Given a simple object $X_{g} \in \mathcal{C}_{g}$ we will denote by $X_{g}^{*} \in \mathcal{C}_{g^{-1}}$ the dual object with respect to the tensor product $\otimes$. Then

$$
\bar{X}_{g}:=X_{g}^{*} \otimes \lambda\left(g, g^{-1}\right)^{*} \in \mathcal{C}_{g^{-1}}^{\lambda}
$$

is also a simple object and $X_{g}^{*} \otimes X_{g} \cong \bar{X}_{g} \stackrel{\lambda}{\otimes} X_{g}$. Hence $\operatorname{Hom}\left(\mathbf{1}, X_{g}^{*} \otimes^{\lambda} X_{g}\right) \neq 0$, and we will use Lemma 3.7 to find specific formulas for the evaluation and coevaluation maps of $X_{g}$ in $\mathcal{C}^{\lambda}$. 
We are assuming that $\lambda(e, g)=\lambda(g, e)=\mathbf{1}$ for all $g \in G$. Hence we have isomorphisms

$$
\lambda_{g}:=\lambda_{g, g^{-1}, g}: \lambda\left(g, g^{-1}\right) \rightarrow \lambda\left(g^{-1}, g\right), \quad g \in G .
$$

As we notice in Example 2.1 any pointed fusion category has a spherical structure. Then for any invertible objects $a \in \mathcal{C}$ in addition to the maps

$$
\epsilon_{a}: a^{*} \otimes a \rightarrow \mathbf{1}, \quad \delta_{a}: \mathbf{1} \rightarrow a \otimes a^{*},
$$

we have maps

$$
\epsilon_{a}^{\prime}: a \otimes a^{*} \rightarrow \mathbf{1}, \quad \delta_{a}^{\prime}: 1 \rightarrow a^{*} \otimes a,
$$

such that

$$
\operatorname{dim}(a)=\epsilon_{a}^{\prime} \circ \delta_{a}=\epsilon_{a} \circ \delta_{a}^{\prime} \in \mathbb{k}^{\times} .
$$

Using these maps, we define $\phi_{X_{g}}: \bar{X}_{g} \stackrel{\lambda}{\otimes} X_{g} \rightarrow \mathbf{1}$ via the following pictures,

$$
\rho_{X_{g}}=\overbrace{X_{g}}^{X_{g}^{*}} \overbrace{X_{g}^{*}}^{\lambda(g)^{*}}
$$

We obtain the scalar

$$
z\left(\phi_{X_{g}}, \rho_{X_{g}}\right)=\operatorname{dim}\left(\lambda\left(g, g^{-1}\right)\right)^{-1} .
$$

Hence for any $X_{g} \in \mathcal{C}_{g}^{\lambda}$ (not necessarily simple) the data

$$
\left(\bar{X}_{g}=X_{g}^{*} \otimes \lambda\left(g, g^{-1}\right)^{*}, \phi_{X_{g}}, \operatorname{dim}\left(\lambda\left(g, g^{-1}\right)\right)^{-1} \rho_{X_{g}}\right)
$$

define a dual in $\mathcal{C}^{\lambda}$, where

$$
\operatorname{dim}\left(\lambda\left(g, g^{-1}\right)\right)=\bigcap^{\lambda\left(g, g^{-1}\right)}=\epsilon_{\lambda\left(g, g^{-1}\right)} \circ \delta_{\lambda(g, g)}^{\prime} .
$$

3.4. Obstruction to associative zestings. Let $G$ be a finite group and $\mathcal{C}$ a $G$ graded fusion category. Recall that $R_{\mathcal{C}_{e}}(\mathcal{C})$ is a braided fusion category. We will denote by $B$ the abelian group $\operatorname{Inv}\left(R_{\mathcal{C}_{e}}(\mathcal{C})\right)$ of isomorphism classes of invertible objects in $R_{\mathcal{C}_{e}}(\mathcal{C})$.

Definition 3.8. (1) We will say that a 2-cocycle $\beta \in Z^{2}(G, B)$ has a lifting if there is a $G$-zesting $\lambda$ of $\mathcal{C}$ such that $\beta(g, h)=[\lambda(g, h)]$ for all $g, h \in G$. 
(2) Two liftings $\lambda_{1}$ and $\lambda_{2}$ are equivalent if there are isomorphisms $f_{g_{1}, g_{2}}: \lambda_{1}\left(g_{1}, g_{2}\right) \rightarrow$ $\lambda_{2}\left(g_{1}, g_{2}\right)$ such that

$$
\left(f_{g_{2}, g_{3}} \otimes f_{g_{1}, g_{2} g_{3}}\right) \circ \lambda_{1}\left(g_{1}, g_{2}, g_{3}\right)=\lambda_{2}\left(g_{1}, g_{2}, g_{3}\right) \circ\left(f_{g_{1}, g_{2}} \otimes f_{g_{1} g_{2}, g_{3}}\right),
$$

for all $g_{1}, g_{2}, g_{3} \in G$.

Since every invertible object is simple, we have that $\operatorname{Aut}_{\mathcal{B}}(X)=\left\{c \operatorname{id}_{X}: c \in \mathbb{k}^{\times}\right\}$. Hence, we can canonically identify $\operatorname{Aut}_{\mathcal{B}}(X)$ with $\mathbb{k}^{\times}$for any invertible object.

Let $\beta \in Z^{2}(G, B)$. Take $\lambda\left(g_{1}, g_{2}\right) \in R_{\mathcal{C}_{e}}(\mathcal{C})$ such that the isomorphism class of $\lambda\left(g_{1}, g_{2}\right)$ is $\beta\left(g_{1}, g_{2}\right)$ and isomorphisms

$$
\lambda_{g_{1}, g_{2}, g_{3}}: \lambda\left(g_{1}, g_{2}\right) \otimes \lambda\left(g_{1} g_{2}, g_{3}\right) \rightarrow \lambda\left(g_{2}, g_{3}\right) \otimes \lambda\left(g_{1}, g_{2} g_{3}\right),
$$

for all $g_{1}, g_{2}, g_{3} \in G$.

Define a map $\nu_{\lambda}: G^{\times 4} \rightarrow \mathbb{k}^{\times}$, where $\nu_{\lambda}\left(g_{1}, g_{2}, g_{3}, g_{4}\right) \in \mathbb{k}^{\times}$is given by the automorphism of $\lambda\left(g_{1}, g_{2}\right) \otimes \lambda\left(g_{1} g_{2}, g_{3}\right) \otimes \lambda\left(g_{1} g_{2} g_{3}, g_{4}\right)$ defined in Figure 5

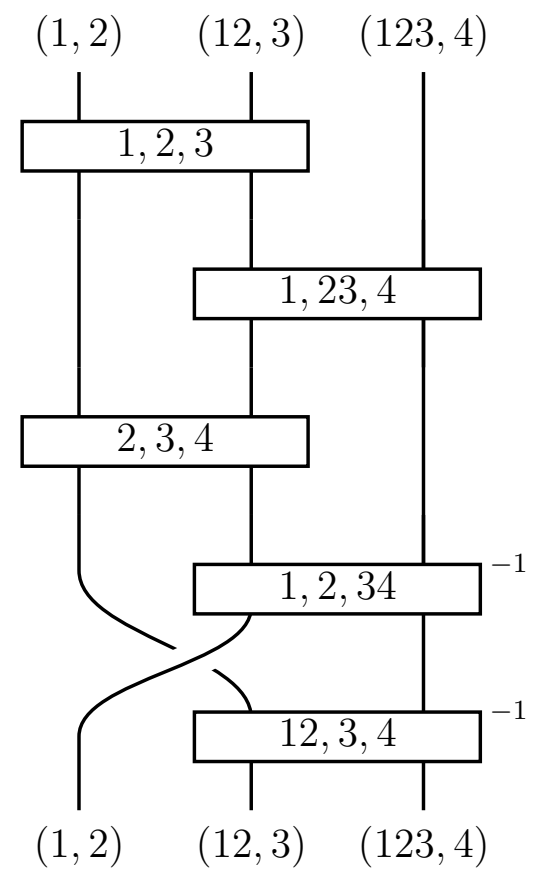

FiguRE 5. 4-cocycle obstruction

Proposition 3.9 ([15]). $\quad$ (i) $\nu_{\lambda} \in Z^{4}\left(A, \mathbb{k}^{\times}\right)$.

(ii) The cohomology class of $\nu_{\lambda}$ only depends on the cohomology class of $\beta$.

(iii) The map $\nu$ induces a map $P W: H^{2}(A, \operatorname{Inv}(\mathcal{B})) \rightarrow H^{4}\left(A, \mathbb{k}^{\times}\right)$.

(iv) The 2-cocycle $\beta$ admits a lifting if and only if $P W(\beta)=0$.

(v) If $P W(\beta)=0$, the set of equivalence classes of lifting is a torsor over $H^{3}\left(A, \mathbb{k}^{\times}\right)$.

Proof. The first three items of the proposition correspond to [15, Proposition 8.10]. Item (iv) is consequence of [15, Proposition 8.9] and item (v) is [15, Proposition 8.15] 
Remark 3.10. If $A$ is cyclic then $H^{4}\left(A, \mathbb{k}^{\times}\right)=0$ so any $\beta$ admits a lifting.

\section{BRAIDED ZESTING}

Recall that if $c$ is a braiding for a monoidal category $\mathcal{B}$, then $c_{X, Y}^{\prime}:=c_{Y, X}^{-1}$ is also a braiding for $\mathcal{B}$. The category $\mathcal{B}$ with the braiding $c^{\prime}$ is denoted $\mathcal{B}^{\text {rev }}$.

If $\mathcal{B}$ is a braided monoidal category and $\mathcal{D} \subset \mathcal{B}$ is a monoidal subcategory, the functors

$$
\begin{aligned}
F: \mathcal{D} & \rightarrow C_{\mathcal{D}}(\mathcal{B}), X \mapsto\left(X,\left\{c_{X, V}\right\}_{V \in \mathcal{B}}\right), \\
G: \mathcal{D}^{r e v} & \rightarrow C_{\mathcal{D}}(\mathcal{B}), X \mapsto\left(X,\left\{c_{X, V}^{\prime}\right\}_{V \in \mathcal{B}}\right)
\end{aligned}
$$

are braided faithful functors.

Definition 4.1. Let $\mathcal{B}$ be a braided faithfully $A$-graded fusion category, where $A$ is an abelian group. A braided zesting consists of a triple $(\lambda, j, t)$, where

(i) $\lambda$ is an associative zesting such that the relative half braiding of $\lambda(a, b)$ is $\left\{c_{\lambda(a, b), V}^{\prime}\right\}_{V \in \mathcal{B}}$ for all $a, b \in A$.

(ii) For any pair $g_{1}, g_{2} \in A$ there is an isomorphism (see Figure 6)

$$
t\left(g_{1}, g_{2}\right): \lambda\left(g_{1}, g_{2}\right) \rightarrow \lambda\left(g_{2}, g_{1}\right),
$$

(iii) A function $j: A \rightarrow \operatorname{Aut}_{\otimes}\left(\operatorname{Id}_{\mathcal{B}}\right)$, where $\operatorname{Aut}_{\otimes}\left(\operatorname{Id}_{\mathcal{B}}\right)$ is the abelian group of all tensor natural isomorphisms of the identity.

The triple $(\lambda, j, t)$, must satisfy the following conditions:

(BZ1) For any $a, b \in A$,

$$
\omega(a, b):=\chi_{\lambda(a, b)} \circ j_{a b} \circ j_{a}^{-1} \circ j_{b}^{-1} \in \operatorname{Aut}_{\otimes}^{A}\left(\operatorname{Id}_{\mathcal{B}}\right) \cong \widehat{A},
$$

and we will denote $\omega(a, b)(c):=\omega(a, b ; c) \in \mathbb{k}^{\times}$.

(BZ2) The equality in Figures 7 and 8 holds, for any $\left(g_{1}, g_{2}, g_{3}\right) \in A^{3}$ and objects $X_{g_{1}} \in \mathcal{B}_{g_{1}}, Y_{g_{2}} \in \mathcal{B}_{g_{2}}, Z_{g_{3}} \in \mathcal{B}_{g_{3}}$.

Moreover, we impose the following normalization conditions:

(a) $t(e, g)=t(g, e)=\operatorname{id}_{\mathbf{1}}$ (Recall that for a normalized associative zesting $\lambda(e, g)=$ 1.)

(b) $j_{e}=$ Id (The identity natural transformation.)

(c) $j_{g}(\mathbf{1})=\mathrm{id}_{\mathbf{1}}$ for all $g \in A$.

Remark 4.2. (1) Condition (BZ1) is equivalent to the condition

$$
\left.\chi_{\lambda(a, b)}\right|_{\mathcal{B}_{e}}=\left(\frac{j_{a} j_{b}}{j_{a b}}\right)_{\mathcal{B}_{e}}
$$

for all $a, b \in \mathcal{B}$.

(2) The choice of the half braiding in braided zesting is compatible with the braidings used in the graphical calculus in the discussion of the relative centralizer from Section 3.1 and Definition 3.2 of associative zesting. 


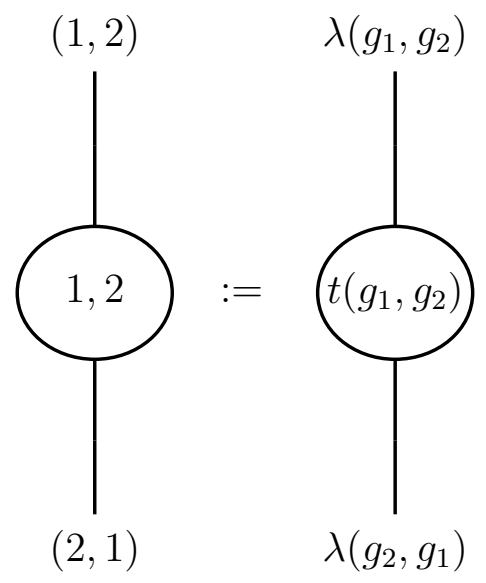

Figure 6. Diagram for the isomorphisms $t\left(g_{1}, g_{2}\right)$.

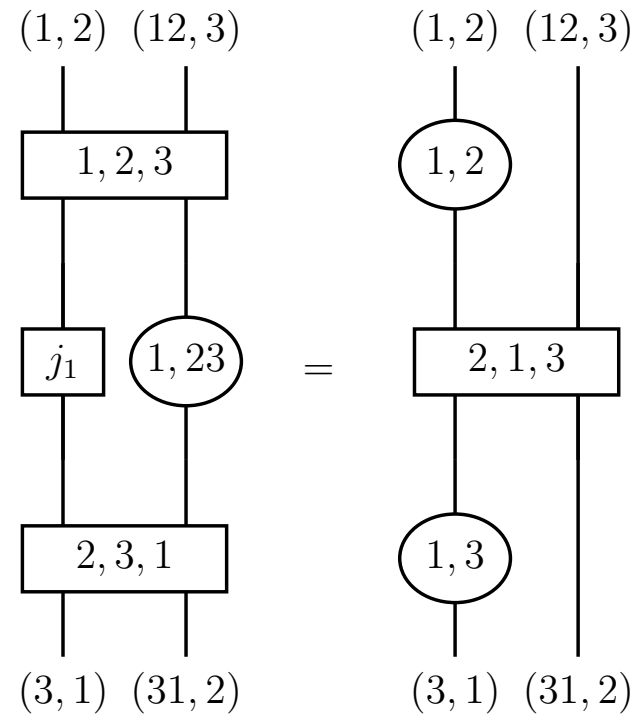

FigURE 7. First braided zesting condition.

(3) Since the two braided zesting conditions are isomorphisms of invertible simple objects, they can be expressed as scalar equations which take the form

$$
\begin{aligned}
j_{1}\left(\lambda\left(g_{2}, g_{3}\right)\right) \lambda_{g_{1}, g_{2}, g_{3}} t\left(g_{1}, g_{2} g_{3}\right) \lambda_{g_{2}, g_{3}, g_{1}} & =t\left(g_{1}, g_{2}\right) \lambda_{g_{2}, g_{1}, g_{3}} t\left(g_{1}, g_{3}\right) \\
\omega(1,2 ; 3) \lambda_{g_{1}, g_{2}, g_{3}}^{-1} t\left(g_{1} g_{2}, g_{3}\right) \lambda_{g_{3}, g_{1}, g_{2}}^{-1} & =t\left(g_{2}, g_{3}\right) \lambda_{g_{1}, g_{3}, g_{2}}^{-1} t\left(g_{1}, g_{3}\right) .
\end{aligned}
$$

Lemma 4.3. For a fixed $\lambda$ and $j$, both $(\lambda, j, t)$ and $\left(\lambda, j, t^{\prime}\right)$ are braided $A$-zestings of $\mathcal{B}$ if and only if $r(a, b):=\frac{t(a, b)}{t^{\prime}(a, b)}$ is a bicharacter on $A$. In particular, such braided zestings form a torsor over the group of bicharacters of $A$. 


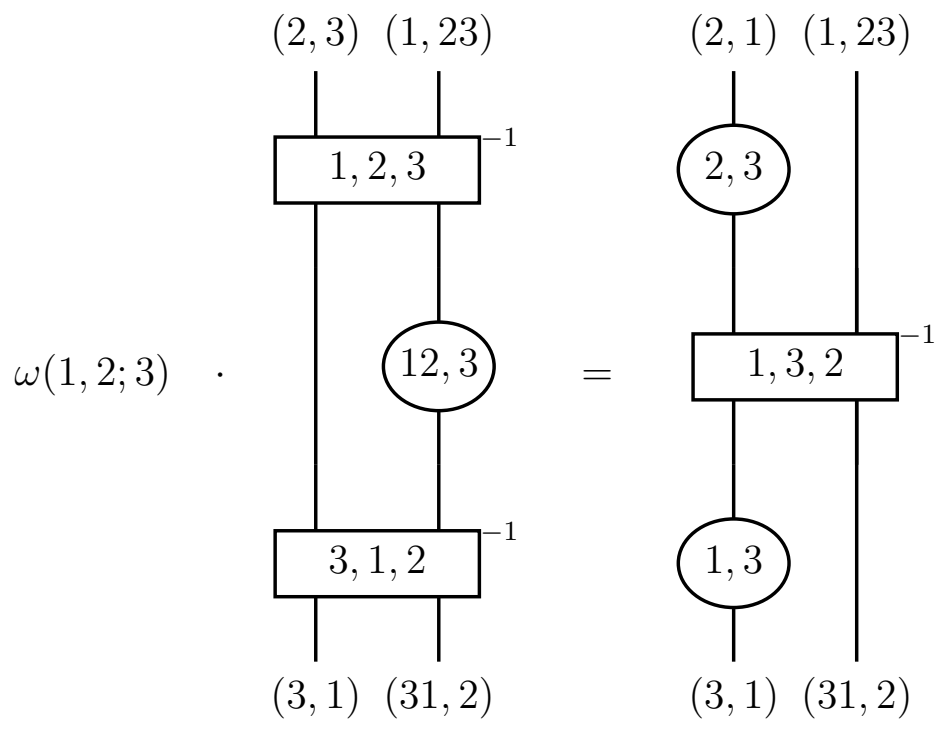

Figure 8. Second braided zesting condition.

Proof. From the form of equations (4.3) and (4.4) we see that $r(a, b+c)=r(a, b) r(a, c)$ and $r(a+b, c)=r(a, c) r(b, c)$.

Proposition 4.4. Let $A$ be an abelian group and $\mathcal{B}$ a faithfully A-graded braided fusion category. Given a braided zesting $(\lambda, j, t)$, the fusion category $\mathcal{B}^{\lambda}$ defined in Proposition 3.4 is braided with braiding $c_{V_{g}, W_{h}}^{(\lambda, j, t)}$ given by the natural isomorphisms defined in Figure 9.
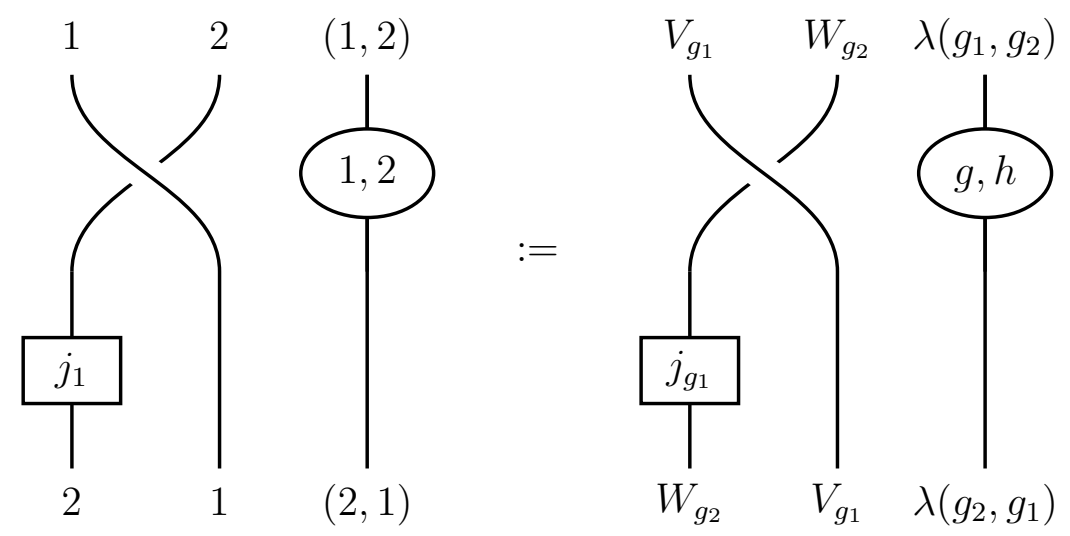

FIGURE 9. Braiding for $\mathcal{B}^{\lambda}$

Proof. The two hexagon equations that must be satisfied by the zested braiding and associators take the form of the equations depicted in Figures 10 and 11, That the equations in Figure 10 and 11 are equivalent to the hexagons can be readily checked in 
the graphical calculus using that the $j_{g}$ 's are monoidal and by applying the definition of $\chi_{a}$ from Figure 1, respectively. These equations are then satisfied by the conditions found in Figures 7 and 8 .

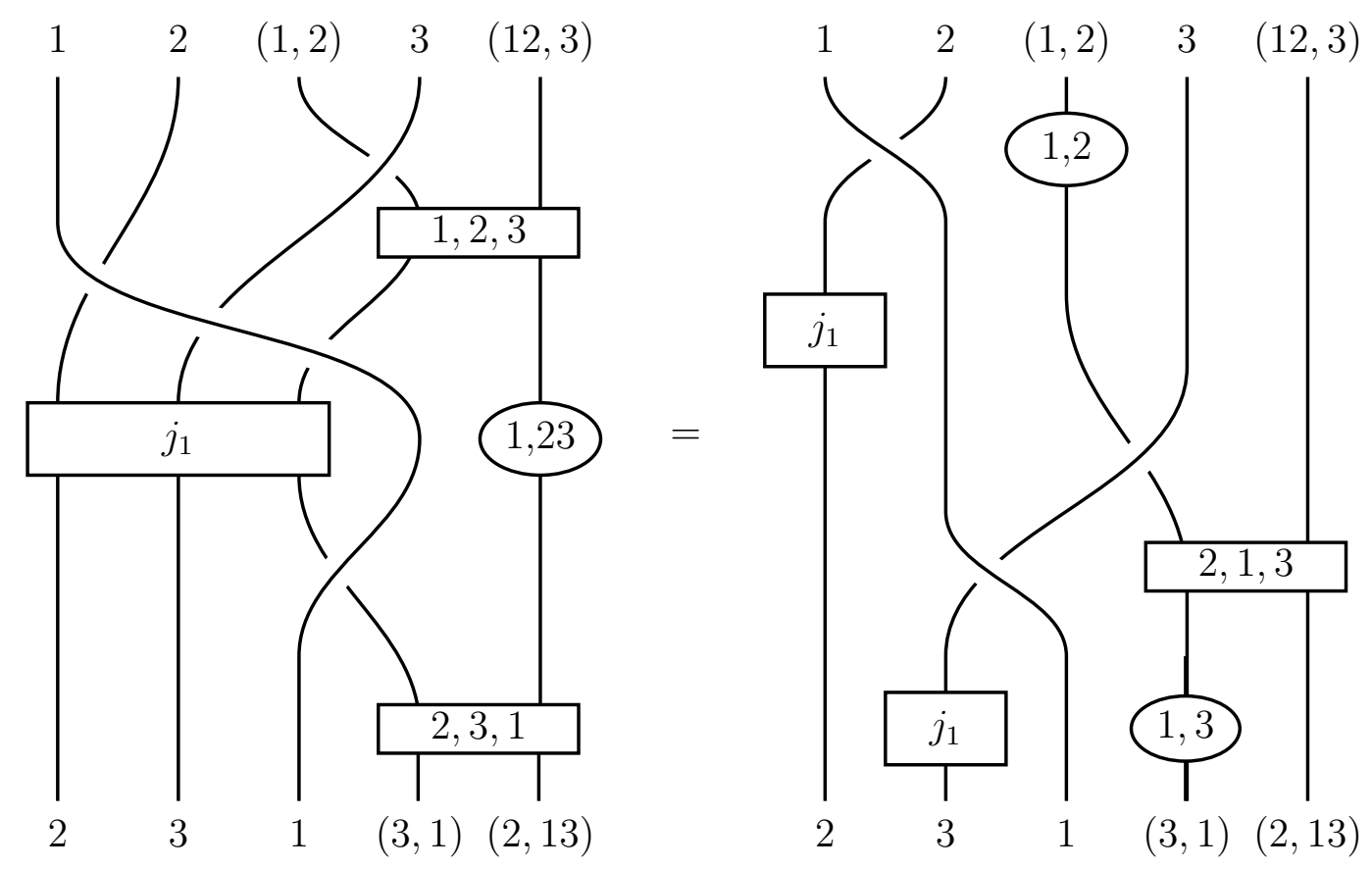

FiguRE 10. First Hexagon condition

Remark 4.5. Notice that a braided zesting does not recover all braidings that may exist on a given associative zesting. For example with the trivial associative zesting (i.e. $\lambda(i, j)=\mathbf{1}$ and $\lambda(i, j, k)=1$ ) on a braided fusion category we might not recover the reverse braiding, as in general this changes the braiding on the trivial component, while braided zesting does not.

4.1. Equivalent braided zesting. Let $A$ be an abelian group and $\mathcal{B}$ a faithfully $A$-graded fusion category.

Definition 4.6. (i) We will say that two braided zestings $(\lambda, j, t)$ and $\left(\lambda, j^{\prime}, t^{\prime}\right)$ are similar if they define the same braiding on $\mathcal{B}^{\lambda}$, that is

$$
c_{X_{a}, Y_{b}}^{(j, t)}=c_{X_{a}, Y_{b}}^{\left(j^{\prime}, t^{\prime}\right)}
$$

for all $a, b \in A, X_{a} \in \mathcal{B}_{a}, Y_{b} \in \mathcal{B}_{b}$.

(ii) We will say that two braided zestings $(\lambda, j, t)$ and $\left(\lambda, j^{\prime}, t^{\prime}\right)$ are braided equivalent if there an alternating character $\nu: A \times A \rightarrow \mathbb{k}^{\times}$such that $(\lambda, j, t)$ is similar to $\left(\lambda, j^{\prime}, \nu t^{\prime}\right)$. 


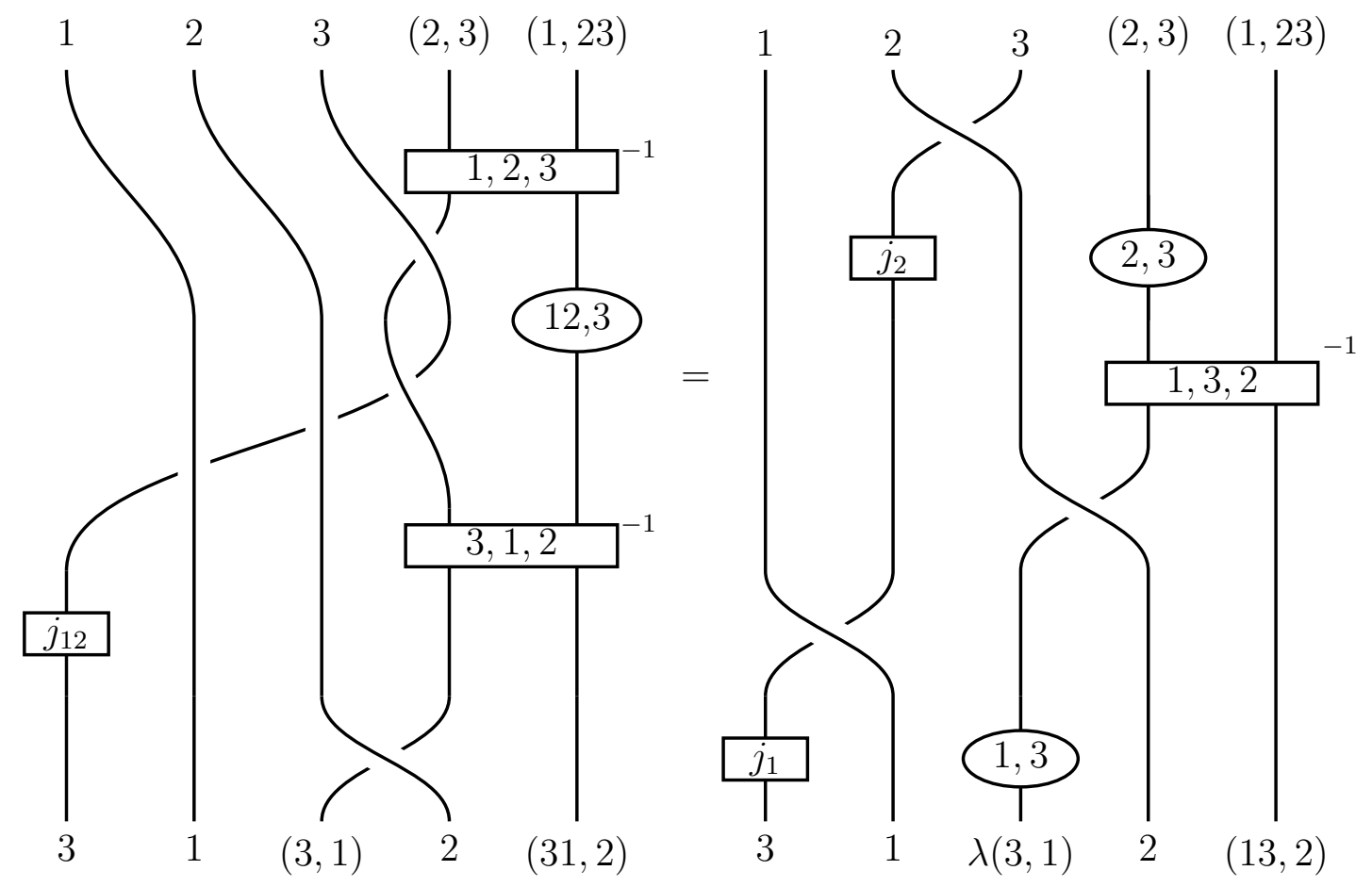

Figure 11. Second Hexagon condition

Let $\lambda$ be an associative $A$-zesting of a braided fusion category $\mathcal{B}$. We define the abelian group

$$
H_{\lambda}=\left\{(\kappa, l) \in C^{1}\left(A, \operatorname{Aut}_{\otimes}\left(\operatorname{Id}_{\mathcal{B}}\right)\right) \times C^{2}\left(A, \mathbb{k}^{\times}\right): \begin{array}{r}
\delta(\kappa)_{a, b} \in \operatorname{Aut}_{\otimes}^{A}\left(\operatorname{Id}_{\mathcal{B}}\right) \\
\kappa(\lambda(a, b))=\frac{l(a, b) l(a, c)}{l(a, b+c)} \\
\delta(\kappa)_{a, b}(c)=\frac{l(a+b, c)}{l(a, c) l(b, c)} \\
\forall a, b, c \in A
\end{array}\right\}
$$

and the abelian subgroups

$$
\text { (4.8) } H_{2}=\left\{(\kappa, l) \in H_{\lambda}: \kappa_{a}=\mathrm{id}, \forall a \in A \text {, and } l \text { is an alternating bicharacter }\right\}
$$

Proposition 4.7. Let $\lambda$ be an associative A-zesting of a braided fusion category $\mathcal{B}$.

(i) The set of all braided $A$-zestings of the form $(\lambda, j, t)$ with $\lambda$ fixed, form a torsor over the abelian group $H_{\lambda}$ defined in (4.6).

(ii) The set of all braided A-zestings similar to $(\lambda, j, t)$ form a torsor over the abelian group $H_{1}$ defined in (4.7). 
(iii) The set of equivalence classes of braided A-zestings form a torsor over $H_{\lambda} / H_{1} H_{2}$, where $\mathrm{H}_{2}$ was defined in (4.8).

Proof. Let $(\lambda, j, t)$ and $\left(\lambda, j^{\prime}, t^{\prime}\right)$ braided $A$-zestings of $\mathcal{B}$. Then $\kappa_{a}:=j_{a}^{\prime} / j_{a}$ and $l(a, b)=t^{\prime}(a, b) / t(a, b)$ for all $a, b \in A$ define an element in $(\kappa, l) \in H_{\lambda}$. In fact, condition (BZ1) implies that $\delta(\kappa)_{a, b}=\kappa_{a} \kappa_{a b}^{-1} \kappa_{b} \in \mathrm{Aut}_{\otimes}^{A}\left(\operatorname{Id}_{\mathcal{B}}\right)$ for all $a, b \in A$, and condition 4.1(BZ2) implies $\kappa_{a}(\lambda(b, c))=\frac{l(a, b) l(a, c)}{l(a, b+c)}$ and $\delta(\kappa)_{a, b}(c)=\frac{l(a b, c)}{l(a, c) l(b, c)}$ for all $a, b, c \in A$.

For item (ii), note that if $c_{X_{a}, Y_{b}}^{(j, t)}=c_{X_{a}, Y_{b}}^{\left(j^{\prime}, t^{\prime}\right)}$ then $\left[j_{a} \circ j_{a}^{\prime-1}\left(X_{b}\right)\right] \otimes\left[t^{\prime}(a, b)^{-1} \circ t(a, b)\right]=$ $\operatorname{id}_{X_{b} \otimes \lambda(a, b)}$ for all $a, b \in A, X_{b} \in \mathcal{B}_{b}$. Then if $(\kappa, l) \in H_{\lambda}$ such that $j=j^{\prime} \kappa, t=t l$, then $\kappa_{a}\left(X_{b}\right)=l(a, b)^{-1}$ for all $a, b \in A$ and $X_{b} \in \mathcal{B}_{b}$.

Item (iii) follows immediately from (i) and (ii).

Corollary 4.8. Let $\mathcal{B}$ be an A-graded braided fusion category. A braided A-zesting $(\lambda, j, t)$ is similar to an A-braided zesting of the form $\left(\lambda, \operatorname{Id}_{\mathcal{B}}, t^{\prime}\right)$ if and only if

$$
\left.\chi_{\lambda(a, b)}\right|_{\mathcal{B}_{e}}=\operatorname{Id}_{\mathcal{B}_{e}},\left.\quad \quad j_{a}\right|_{\mathcal{B}_{e}}=\operatorname{Id}_{\mathcal{B}_{e}}
$$

for all $a, b \in A$. In particular, if $A$ is the universal grading every braided zesting is similar to a braided zesting of the form $\left(\lambda, \operatorname{Id}_{\mathcal{B}}, t\right)$.

Proof. A braided $A$-zesting $(\lambda, j, t)$ is similar to one of the form $\left(\lambda, \mathrm{id}, t^{\prime}\right)$ if and only if $\left(j^{-1}, l_{j}\right) \in H_{1}$, where $l_{j}(a, b)=j_{a}\left(X_{b}\right)^{-1}$ with $X_{b} \in \mathcal{B}_{b}$. Hence

$$
j_{a} \in \operatorname{Aut}_{\otimes}^{A}\left(\operatorname{Id}_{\mathcal{B}}\right), \quad \forall a \in A,
$$

and (4.10) imply that $\chi_{\lambda(a, b)} \in \mathrm{Aut}_{\otimes}^{A}\left(\operatorname{Id}_{\mathcal{B}}\right)$ or equivalently $\left.\chi_{\lambda(a, b)}\right|_{\mathcal{B}_{e}}=\operatorname{Id}_{\mathcal{B}_{e}}$. Conversely, if conditions (4.10) holds, then it is is easy to see that $\left(j^{-1}, l_{j}\right) \in H_{1}$.

Now, if $A$ is the universal grading group it follows from Proposition 2.7 and Proposition 2.8 that any braided zesting satisfies the condition in (4.9).

4.2. Obstructions to braided zestings. Let $\mathcal{B}$ be a braided fusion category. From now on, using Proposition 2.7 we will identify the abelian groups $\mathrm{Aut}_{\otimes}^{G}\left(\operatorname{Id}_{\mathcal{B}}\right)$ and $\widehat{G}$. Recall that in particular, $\mathrm{Aut}_{\otimes}\left(\operatorname{Id}_{\mathcal{B}}\right) \cong \widehat{U(\mathcal{B})}$.

Let $\mathcal{B}$ be a braided fusion category graded by a finite abelian group $A$. Let $U(\mathcal{B})$ be the universal grading group of $\mathcal{B}$ and $\pi_{1}: U(\mathcal{B}) \rightarrow A$ the group epimorphism that defines the $A$-grading on $\mathcal{B}$. We first describe obstructions to the existence of braided zestings obtained by restricting to the trivially graded component. By restriction of the $U(\mathcal{B})$-grading, the fusion subcategory $\mathcal{B}_{e}$ is $\operatorname{ker}\left(\pi_{1}\right)$-graded with $\left(\mathcal{B}_{e}\right)_{e}=\mathcal{B}_{\text {ad }}$. Then this grading defines a group epimorphism

$$
\pi_{2}: U\left(\mathcal{B}_{e}\right) \rightarrow \operatorname{ker}\left(\pi_{1}\right) .
$$

Proposition 4.9. A tensor natural isomorphism $j \in \operatorname{Aut}_{\otimes}\left(\operatorname{Id}_{\mathcal{B}_{e}}\right) \cong \widehat{U\left(\mathcal{B}_{e}\right)}$ has an extension to an element in $\mathrm{Aut}_{\otimes}\left(\operatorname{Id}_{\mathcal{B}}\right) \cong \widehat{U(\mathcal{B})}$ if and only if $j \in \operatorname{Aut}_{\otimes}^{\operatorname{ker}\left(\pi_{1}\right)}\left(\operatorname{Id}_{\mathcal{B}_{e}}\right)$. The set of extensions of $j$ is a torsor over $\widehat{A}$. 
Proof. We have the exact sequence of abelian groups

$$
0 \rightarrow \operatorname{ker}\left(\pi_{2}\right) \rightarrow U\left(\mathcal{B}_{e}\right) \stackrel{\pi_{2}}{\rightarrow} \operatorname{ker}\left(\pi_{1}\right) \rightarrow U(\mathcal{B}) \stackrel{\pi_{7}}{\rightarrow} A \rightarrow 0
$$

and dualizing

$$
0 \rightarrow \widehat{A} \stackrel{\pi_{*}^{*}}{\rightarrow} \widehat{U(\mathcal{B})} \rightarrow \widehat{\operatorname{ker}\left(\pi_{1}\right)} \stackrel{\pi_{2}^{*}}{\rightarrow} \widehat{U\left(\mathcal{B}_{e}\right)} \rightarrow \widehat{\operatorname{ker}\left(\pi_{2}\right)} \rightarrow 0 .
$$

Hence the image of the restriction map $\widehat{U(\mathcal{B})} \rightarrow \widehat{U\left(\mathcal{B}_{e}\right)}$ is exactly $\widehat{\operatorname{ker}\left(\pi_{1}\right)}$, or equivalently all $\gamma \in U\left(\mathcal{B}_{e}\right)$ such that $\left.\gamma\right|_{\operatorname{ker}\left(\pi_{2}\right)} \equiv 1$.

It follows from Proposition 4.9 that a first partial obstruction to the existence of a braided zesting is that

$$
\left.\chi_{\lambda(a, b)}\right|_{\mathcal{B}_{e}} \in \operatorname{Aut}_{\otimes}^{\operatorname{ker}\left(\pi_{1}\right)}\left(\operatorname{Id}_{\mathcal{B}_{e}}\right) \cong \widehat{\operatorname{ker}\left(\pi_{1}\right)}, \quad \forall a, b \in A .
$$

It follows from Proposition 2.7 that condition (4.12) is equivalent to

$$
\chi_{\lambda(a, b)}(X)=\operatorname{id}_{X}, \quad \text { for all } X \in\left(\mathcal{B}_{e}\right)_{g} \text { where } g \in \operatorname{ker}\left(\pi_{2}\right) \subset U\left(\mathcal{B}_{e}\right),
$$

where $\pi_{2}$ was defined in (4.11).

It follows by condition (BZ1) that

$$
\left.\chi_{\lambda(a, b)}\right|_{\mathcal{B}_{e}}=\left(j_{a} \circ j_{b} \circ j_{a b}^{-1}\right)_{\mathcal{B}_{e}},
$$

where $j$ 's are in $\operatorname{Aut}_{\otimes}^{\operatorname{ker}\left(\pi_{1}\right)}\left(\operatorname{Id}_{\mathcal{B}_{e}}\right)$. Hence a second partial obstruction is that the cohomology class of

$$
\left.\chi_{\lambda(-,-)}\right|_{\mathcal{B}_{e}} \in H^{2}\left(A, \operatorname{Aut}_{\otimes}^{\operatorname{ker}\left(\pi_{1}\right)}\left(\operatorname{Id}_{\mathcal{B}_{e}}\right)\right)=H^{2}\left(A, \widehat{\operatorname{ker}\left(\pi_{1}\right)}\right),
$$

must be trivial.

Remark 4.10. When $A$ is the universal grading the first and second partial obstructions automatically vanish. If $A$ is the universal grading and $\mathcal{B}$ is modular then $\operatorname{ker}\left(\pi_{2}\right)=U\left(\mathcal{B}_{e}\right)$. Since $\pi_{1}$ is trivial, the grading on $\mathcal{B}_{e}$ is trivial, so $\left(\mathcal{B}_{e}\right)_{g}=\mathcal{B}_{e}=\mathcal{B}_{a d}$. Therefore $\lambda(a, b) \in \mathcal{B}_{p t}$ centralizes $\mathcal{B}_{e}, \chi_{\lambda(a, b)}(X)=\operatorname{id}_{X}$, and the first partial obstruction vanishes. The triviality of $\operatorname{ker}\left(\pi_{1}\right)$ implies that the second partial obstruction vanishes.

4.2.1. Shuffle identities. In this section we collect some notation and identities that will be useful later.

We use the following notation, where $A$ is a group:

(a) $A^{p} \mid A^{q}=\left\{\boldsymbol{x} \mid \boldsymbol{y}=\left(x_{1}, \ldots, x_{p} \mid y_{1}, \ldots, y_{q}\right), x_{i}, y_{j} \in A\right\}, p, q \geq 0$.

(b) $\operatorname{Shuff}(p, q)$ is the set of $(p, q)$-shuffles, i.e. elements in the symmetric group $\mathbb{S}_{p+q}$ such that $\lambda(i)<\lambda(j)$ whenever $1 \leq i<j \leq p$ or $p+1 \leq i<j \leq p+q$.

Now let $A$ and $N$ be abelian groups. We define a double complex by $D^{p, q}(A, N)=0$ if $p$ or $q$ is zero and

$$
D^{p, q}(A, N):=\operatorname{Maps}\left(A^{p} \mid A^{q} ; N\right), \quad p, q>0
$$

with horizontal and vertical differentials the standard differentials, that is,

$$
\delta_{h}: D^{p, q}(A, N)=C^{p}\left(A, C^{q}(A, N)\right) \rightarrow D^{p+1, q}(A, N)=C^{p+1}\left(A, C^{q}(A, N)\right)
$$


and

$$
\delta_{v}: D^{p, q}(A, N)=C^{q}\left(A, C^{p}(A, N)\right) \rightarrow D^{p, q+1}(A, N)=C^{q+1}\left(A, C^{p}(A, N)\right)
$$

defined by the equations

$$
\begin{aligned}
\left(\delta_{h} F\right)\left(g_{1}, \ldots, g_{p+1} \mid k_{1}, \ldots, k_{q}\right)=F\left(g_{2}, \ldots,\right. & \left.g_{p+1} \mid k_{1}, \ldots, k_{q}\right) \\
& +\sum_{i=1}^{p}(-1)^{i} F\left(g_{1}, \ldots, g_{i} g_{i+1}, . ., g_{p+1} \mid k_{1}, \ldots, k_{q}\right) \\
& +(-1)^{p+1} F\left(g_{1}, \ldots, g_{p} \mid k_{1}, \ldots, k_{q}\right) \\
\left(\delta_{v} F\right)\left(g_{1}, \ldots, g_{p} \mid k_{1}, \ldots, k_{q+1}\right)=F\left(g_{1}, \ldots, g_{p} \mid k_{2}, \ldots, k_{q+1}\right) & \\
& +\sum_{j=1}^{q}(-1)^{j} F\left(g_{1}, \ldots, g_{p} \mid k_{1}, \ldots, k_{j} k_{j+1}, \ldots, k_{q+1}\right) \\
& +(-1)^{q+1} F\left(g_{1}, \ldots, g_{p} \mid k_{1}, \ldots, k_{q}\right) .
\end{aligned}
$$

For any $\alpha \in C^{n}(A, N)$, for any $1 \leq p \leq n-1$ we define the $p$-th shuffle $\alpha_{p} \in$ $\operatorname{Maps}\left(A^{p} \mid A^{n-p}, N\right)$ as

$$
\alpha_{p}\left(a_{1}, \ldots, a_{p} \mid a_{p+1}, \ldots, a_{n}\right)=\sum_{\pi \in \operatorname{Shuff}(\mathrm{p}, \mathrm{n}-\mathrm{p})}(-1)^{\epsilon(\pi)} \alpha\left(a_{\pi(1)}, \ldots, a_{\pi(n)}\right) .
$$

Proposition 4.11. [20, Proposition 2, page 123] Let $\alpha \in C^{n}(A, N)$ then

$$
(\delta \alpha)_{p}=\delta_{h}\left(\alpha_{p-1}\right)+(-1)^{p} \delta_{v}\left(\alpha_{p}\right) .
$$

for all $1 \leq p \leq n$, where by notation $\alpha_{0}=\alpha_{n}=0$, and $\delta$ is the standard differential (2.1).

4.2.2. General obstructions. Now let us consider the more general obstruction theory.

Fix an associative zesting $\lambda$ of $\mathcal{B}$ (i.e., a 2-cocycle $\lambda \in H^{2}\left(A, \operatorname{Inv}\left(\mathcal{B}_{e}\right)\right)$ and isomorphisms $\lambda$ satisfying Definition 3.2) such that the first and second partial obstructions vanish.

In order to solve the equation in Figure 7 we fix an arbitrary family of isomorphisms $\{\nu(a, b): \lambda(a, b) \rightarrow \lambda(b, a)\}_{a, b \in A}$ (see Figure [12) and a map $\tilde{j}: A \rightarrow \operatorname{Aut}_{\otimes}^{\operatorname{ker}\left(\pi_{1}\right)}\left(\operatorname{Id}_{\mathcal{B}_{e}}\right)$ such that $\left.\chi_{\lambda(a, b)}\right|_{\mathcal{B}_{e}}=\tilde{j}_{a} \circ \tilde{j}_{b} \circ \tilde{j}_{a b}^{-1}$. Then we define a map $O_{1}(\lambda, \nu, \tilde{j}) \in \operatorname{Maps}\left(A^{2} \mid A, \mathbb{k}^{\times}\right)$,

$$
\begin{aligned}
O_{1}(\lambda, \nu, \tilde{j}): A \times A \times A & \rightarrow \mathbb{k}^{\times} \\
\left(a_{1}, a_{2}, a_{3}\right) & \mapsto O_{1}\left(a_{1} \mid a_{2}, a_{3}\right)
\end{aligned}
$$

by Figure 13 .

Lemma 4.12. (i) For any $a \in A$ the function $O_{1}(\lambda, \nu, \tilde{j})(a \mid-,-): A \times A \rightarrow \mathbb{k}^{\times}$ defines a 2-cocycle.

(ii) The cohomology class $O_{1}(\lambda, \nu, \tilde{j})(a \mid-,-)$ does not depend on the choice of the family $\nu$ or $\tilde{j}$, and we will be denoted by $O_{1}(\lambda)(a \mid-,-)$.

(iii) There is a choice of isomorphisms $\nu$ that satisfies the equation in Figure 7 if and only if the cohomology class of $O_{1}(\lambda)(a \mid-,-) \in H^{2}\left(A, \mathbb{k}^{\times}\right)$vanishes for each $a \in A$. 


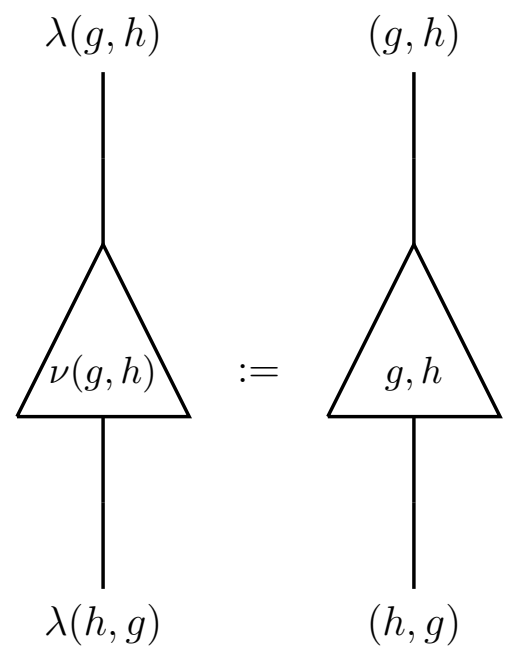

Figure 12. Diagram for the isomorphisms $\nu(g, h)$.

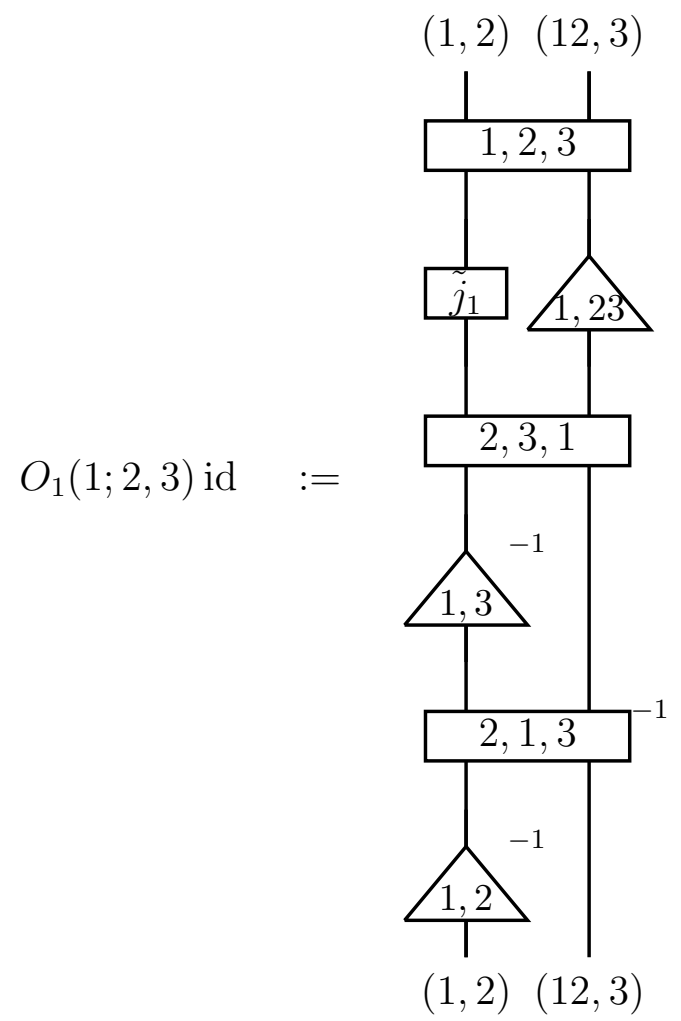

Figure 13. Obstruction to Figure 7

Proof. To simplify the arguments and notation we will assume without serious loss of generality that

$$
\begin{array}{r}
\lambda\left(a_{1}, a_{2}\right) \otimes \lambda\left(a_{1} a_{2}, a_{3}\right)=\lambda\left(a_{2}, a_{3}\right) \otimes \lambda\left(a_{2} a_{3}, a_{1}\right) \\
\lambda\left(a_{1}, a_{2}\right)=\lambda\left(a_{2}, a_{1}\right), \quad \lambda\left(a_{1}, a_{2}\right) \otimes \lambda\left(a_{3}, a_{4}\right)=\lambda\left(a_{3}, a_{4}\right) \otimes \lambda\left(a_{1}, a_{2}\right)
\end{array}
$$


for all $a_{1}, a_{2}, a_{3}, a_{4} \in A$. Hence the isomorphisms $\lambda\left(a_{1}, a_{2}, a_{3}\right)$ and $\nu\left(a_{1}, a_{2}\right)$ are defined by cochains $\lambda \in C^{3}\left(A, \mathbb{k}^{\times}\right), \nu \in C^{2}\left(A, \mathbb{k}^{\times}\right)$and the associative zesting constraint can be written as

$$
\delta(\lambda)=\left(\lambda \cup_{c^{\prime}} \lambda\right)
$$

where $\left(\lambda \cup_{c^{\prime}} \lambda\right)\left(a_{1}, a_{2}, a_{3}, a_{4}\right) \in \mathbb{k}^{\times}$is defined by

$$
\left(\lambda \cup_{c^{\prime}} \lambda\right)\left(a_{1}, a_{2}, a_{3}, a_{4}\right) \operatorname{id}_{\lambda\left(a_{1}, a_{2}\right) \otimes \lambda\left(a_{3}, a_{4}\right)}=c_{\lambda\left(a_{3}, a_{4}\right), \lambda\left(a_{1}, a_{2}\right)}^{-1} .
$$

(i) We have that

$$
\begin{aligned}
O_{1}\left(a_{1} ; a_{2}, a_{3}\right) & =\tilde{j}_{a_{1}}\left(\lambda\left(a_{2}, a_{3}\right)\right) \times \frac{\nu\left(a_{1}, a_{2}+a_{3}\right)}{\nu\left(a_{1}, a_{2}\right) \nu\left(a_{1}, a_{3}\right)} \times \frac{\lambda\left(a_{1}, a_{2}, a_{3}\right) \lambda\left(a_{2}, a_{3}, a_{1}\right)}{\lambda\left(a_{2}, a_{1}, a_{3}\right)} \\
& =\tilde{j}_{a_{1}}\left(\lambda\left(a_{2}, a_{3}\right)\right) \times \delta_{v}\left(\nu^{-1}\right)\left(a_{1} \mid a_{2}, a_{3}\right) \lambda_{1}\left(a_{1} \mid a_{2}, a_{3}\right)
\end{aligned}
$$

for all $a_{1}, a_{2}, a_{3} \in A$, where we have been using the notation introduced in Section 4.2.1. Note that the 2-cochains $\left(a_{2}, a_{2}\right) \mapsto \tilde{j}_{a_{1}}\left(\lambda\left(a_{2}, a_{3}\right)\right)$ and $\left(a_{2}, a_{3}\right) \mapsto \delta_{h}(\nu)\left(a_{1} \mid a_{2}, a_{3}\right)$ are 2-cocycles. Hence, to prove that $O_{1}\left(a_{1} ;-,-\right)$ is a 2-cocycle we only need to check that

$$
\delta_{v}\left(\lambda_{1}\right)\left(a_{1} \mid a_{2}, a_{3}, a_{4}\right)=1 \quad \forall a_{1}, a_{2}, a_{3}, a_{4} \in A .
$$

It follows from Proposition 4.11 that

$$
\begin{aligned}
\delta_{v}\left(\lambda_{1}\right)\left(a_{1} \mid a_{2}, a_{3}, a_{4}\right)^{-1}= & \prod_{\pi \in \operatorname{Shuff}(1,3)} \delta(\lambda)\left(a_{\pi(1)}, \ldots, a_{\pi(4)}\right)^{\epsilon(\pi)} \\
= & \frac{\left(\lambda \cup_{c^{\prime}} \lambda\right)\left(a_{1}, a_{2}, a_{3}, a_{4}\right)\left(\lambda \cup_{c^{\prime}} \lambda\right)\left(a_{2}, a_{3}, a_{4}, a_{1}\right)}{\left(\lambda \cup_{c^{\prime}} \lambda\right)\left(a_{2}, a_{1}, a_{3}, a_{4}\right)\left(\lambda \cup_{c^{\prime}} \lambda\right)\left(a_{2}, a_{3}, a_{1}, a_{4}\right)} \\
& =1,
\end{aligned}
$$

where the last equality follows because $\lambda(a, b)=\lambda(b, a)$ for all $a, b \in A$.

(ii) Recall that every symmetric 2-cocycle in $Z^{2}\left(A, \mathbb{k}^{\times}\right)$is a coboundary (since $\mathbb{k}^{\times}$ is divisible). Then $\delta_{v}\left(\nu^{-1}\right)\left(a_{1} \mid-,-\right)$ and $\tilde{j}_{a_{1}}(\lambda(-,-))$ contributes with a 2-coboundary to $O_{1}(\lambda, \nu, t)\left(a_{1} \mid-,-\right)$. Hence the cohomology class $O_{1}\left(a_{1} \mid-,-\right)$ only depends on $\lambda$.

(iii) If there is $h \in C^{2}\left(A, \mathbb{k}^{\times}\right)$such that $\delta_{v}(h)=O_{1}(\lambda, \nu, \tilde{j})$, then taking $\nu^{\prime}=\nu h^{-1}$ we have that $O_{1}\left(\lambda, \nu^{\prime}, \tilde{j}\right)=1$.

Remark 4.13. In practice we can take $\lambda(a, b)=\lambda(b, a)$ for all $a, b \in A$. Since $\lambda(a, b)$ is invertible we have that every isomorphism $\nu: \lambda(a, b) \rightarrow \lambda(b, a)$ is a multiple of the identity. Hence in order to compute $O_{1}(\lambda)$, we can start with $\nu(a, b)=\operatorname{id}_{\lambda(a, b)}$ for all $a, b \in A$.

Assuming that the cohomology classes of $O_{1}(\lambda)$ vanish, we can find isomorphisms $\nu: \lambda(a, b) \rightarrow \lambda(b, a)$ such that $O_{1}(\lambda, \nu, j)\left(a_{1} \mid a_{2}, a_{3}\right)=1$ for all $a_{1}, a_{2}, a_{3} \in A$. We define the map $O_{2}(\lambda, \mu, j)(a \mid b, c) \in \mathbb{k}^{\times}$by Figure 14 . 


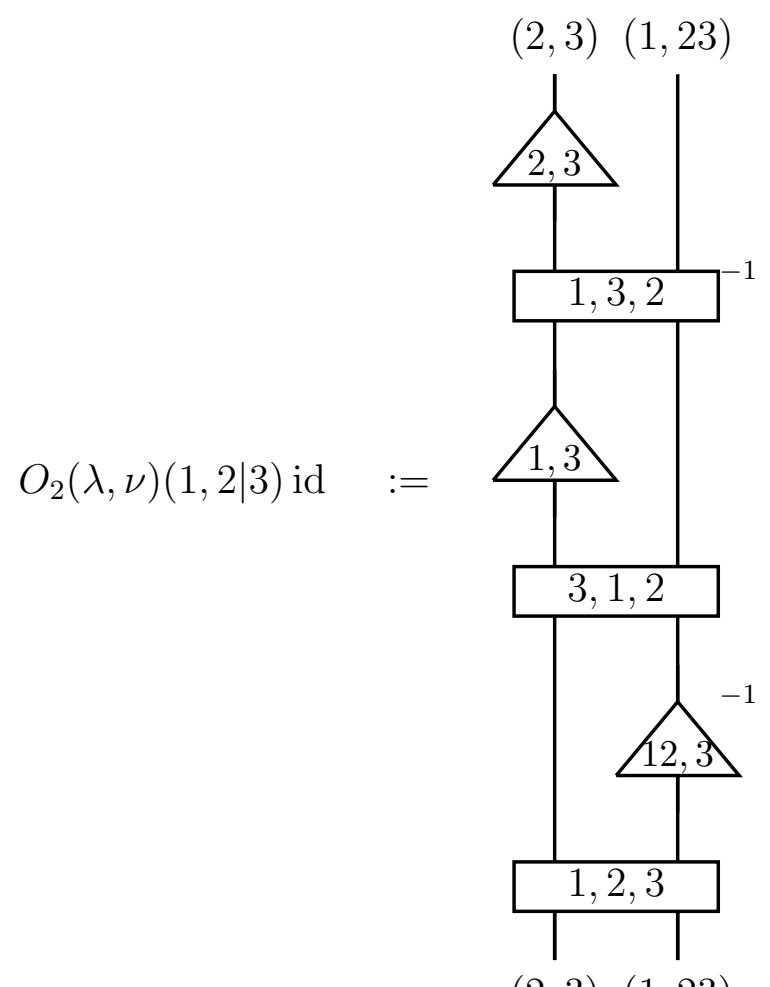

Figure 14. Diagrammatic definition of the second obstruction to braided zesting.

Lemma 4.14. Let $\tilde{j}: A \rightarrow \operatorname{Aut}_{\otimes}^{\operatorname{ker}\left(\pi_{1}\right)}\left(\operatorname{Id}_{\mathcal{B}}\right)$ be map such that $\delta(\tilde{j})(a, b)=\left.\chi_{\lambda(a, b)}\right|_{\mathcal{B}_{e}}$ for all $a, b \in A$ and let $\nu: \lambda(a, b) \rightarrow \lambda(b, a)$ be a family of isomorphisms such that

$$
O_{1}(\lambda, \nu, \tilde{j})\left(a_{1} \mid a_{2}, a_{3}\right)=1, \quad \forall a_{1}, a_{2}, a_{3} \in A .
$$

Then

(i) $O_{2}(\lambda, \nu)(-\mid a, b) \in \widehat{A}$ for all $a, b \in A$.

(ii) The 2-cochain

$$
\begin{aligned}
O_{2}(\lambda, \nu): A \times A & \rightarrow \widehat{A} \\
(a, b) & \mapsto\left[c \mapsto O_{2}(\lambda)(c \mid a, b)\right]
\end{aligned}
$$

defines a 2-cocycle $O_{2}(\lambda, \nu) \in Z^{2}(A, \widehat{A})$. The cohomology class of $O_{2}(\lambda, \nu)$ does not depend on the choice of the $\nu$ (under the hypothesis that the $O_{1}(\lambda, \nu)=1$ ), and will be denoted by $\mathrm{O}_{2}(\lambda)$.

Proof. As in the proof of Lemma 4.12, we will assume that $\lambda(a, b, c)$ and $\nu(a, b)$ are defined by cochains $\lambda \in C^{3}\left(A, \mathbb{k}^{\times}\right), \nu \in C^{2}\left(A, \mathbb{k}^{\times}\right)$.

The condition $O_{1}(\lambda, \nu, \tilde{j})=1$ can be written as

$$
\delta_{v}(\nu)\left(a_{1} \mid a_{2}, a_{3}\right)=\lambda_{1}\left(a_{1} \mid a_{2}, a_{3}\right) \tilde{j}_{a_{1}}\left(\lambda\left(a_{2}, a_{3}\right)\right), \quad \forall a_{1}, a_{2}, a_{3} \in A .
$$


(i) We need to check that $\delta_{v}\left(O_{2}(\lambda, \nu, \tilde{j})\right)\left(a_{1}, a_{2} \mid a_{3}, a_{4}\right)=1$, where

$$
O_{2}(\lambda, \nu, \tilde{j})\left(a_{1}, a_{2} \mid a_{3}\right)=\delta_{h}(\nu)\left(a_{1}, a_{2} \mid a_{3}\right) \lambda_{2}\left(a_{1}, a_{2} \mid a_{3}\right) .
$$

First we have that

$$
\begin{aligned}
\delta_{v}\left(\delta_{h}(v)\right)\left(a_{1}, a_{2} \mid a_{3}, a_{4}\right) & =\delta_{h}\left(\delta_{v}(v)\right)\left(a_{1}, a_{2} \mid a_{3}, a_{4}\right) \\
& =\delta_{h}\left(\lambda_{1}\right)\left(a_{1}, a_{2} \mid a_{3}, a_{4}\right) \tilde{j}_{a_{1}} \tilde{j}_{a_{1}+a_{2}}^{-1} \tilde{j}_{a_{2}}\left(\lambda\left(a_{3}, a_{4}\right)\right) \\
& =\delta_{h}\left(\lambda_{1}\right)\left(a_{1}, a_{2} \mid a_{3}, a_{4}\right) \chi_{\lambda\left(a_{1}, a_{2}\right)}\left(\lambda\left(a_{3}, a_{4}\right)\right) .
\end{aligned}
$$

and

$$
\begin{aligned}
\delta_{v}\left(O_{2}(\lambda, \nu, \tilde{j})\right)\left(a_{1}, a_{2} \mid a_{3}, a_{4}\right) & =\delta_{v}\left(\delta_{h}(v)\right) \delta_{v}\left(\lambda_{2}\right)\left(a_{1}, a_{2} \mid a_{3}, a_{4}\right) \\
& =\left(\delta_{h}\left(\lambda_{1}\right) \delta_{v}\left(\lambda_{2}\right)\right)\left(a_{1}, a_{2} \mid a_{3}, a_{4}\right) \chi_{\lambda\left(a_{1}, a_{2}\right)}\left(\lambda\left(a_{3}, a_{4}\right)\right) .
\end{aligned}
$$

Using Proposition 4.11 we have

$$
\begin{aligned}
\delta_{h}\left(\lambda_{1}\right)\left(a_{1}, a_{2} \mid a_{3}, a_{4}\right) \delta_{v}\left(\lambda_{2}\right)\left(a_{1}, a_{2} \mid a_{3}, a_{4}\right)= & \prod_{\pi \in \operatorname{Shuff}(2,2)} \delta(\lambda)\left(a_{\pi(1)}, \ldots, a_{\pi(4)}\right)^{\epsilon(\pi)} \\
= & \lambda \cup_{c^{\prime}} \lambda\left(a_{1}, a_{2}, a_{3}, a_{4}\right) \lambda \cup_{c^{\prime}} \lambda\left(a_{3}, a_{4}, a_{1}, a_{2}\right) \\
& \lambda \cup_{c^{\prime}} \lambda\left(a_{3}, a_{1}, a_{2}, a_{4}\right) \lambda \cup_{c^{\prime}} \lambda\left(a_{1}, a_{3}, a_{2}, a_{4}\right) \\
& \lambda \cup_{c^{\prime}} \lambda\left(a_{3}, a_{1}, a_{4}, a_{2}\right)^{-1} \lambda \cup_{c^{\prime}} \lambda\left(a_{1}, a_{3}, a_{4}, a_{2}\right)^{-1} \\
= & \lambda \cup_{c^{\prime}} \lambda\left(a_{1}, a_{2}, a_{3}, a_{4}\right) \lambda \cup_{c^{\prime}} \lambda\left(a_{3}, a_{4}, a_{1}, a_{2}\right) \\
= & \chi_{\lambda\left(a_{1}, a_{2}\right)}^{-1}\left(\lambda\left(a_{3}, a_{4}\right)\right) .
\end{aligned}
$$

Then

$$
\delta_{v}\left(O_{2}(\lambda, \nu, \tilde{j})\right)\left(a_{1}, a_{2} \mid a_{3}, a_{4}\right)=1
$$

as we wanted to check.

(ii) The 2-cocycle condition in this case is

$$
\delta_{h}\left(O_{2}(\lambda, \nu, \tilde{j})\right)=\delta_{h}\left(\lambda_{2}\right)=1 .
$$

Using again Proposition 4.11 we have

$$
\begin{aligned}
\delta_{h}\left(\lambda_{2}\right)= & \prod_{\pi \in \operatorname{Shuff}(3,1)} \delta(\gamma)\left(a_{\pi(1)}, \ldots, a_{\pi(4)}\right)^{\epsilon(\pi)} \\
= & \lambda \cup_{c^{\prime}} \lambda\left(a_{1}, a_{2}, a_{3}, a_{4}\right) \lambda \cup_{c^{\prime}} \lambda\left(a_{4}, a_{1}, a_{2}, a_{3}\right) \\
& \lambda \cup_{c^{\prime}} \lambda\left(a_{1}, a_{4}, a_{2}, a_{3}\right)^{-1} \lambda \cup_{c^{\prime}} \lambda\left(a_{1}, a_{2}, a_{4}, a_{3}\right)^{-1} \\
= & 1 .
\end{aligned}
$$

Finally, if $\nu^{\prime}$ is another 2-cochain such that $O_{1}\left(\lambda, \nu^{\prime}, \tilde{j}\right)=1$, then $\delta_{v}\left(\nu / \nu^{\prime}\right)=1$, that is $\nu / \nu^{\prime} \in C^{1}(A, \widehat{A})$, and then $O_{2}\left(\lambda, \nu^{\prime}, \tilde{j},\right)=\delta_{h}\left(\nu / \nu^{\prime}\right) O_{2}\left(\lambda, \nu^{\prime}, \tilde{j},\right)$, that is $O_{2}\left(\lambda, \nu^{\prime}, \tilde{j},\right)$ and $O_{2}(\lambda, \nu, \tilde{j}$,$) are cohomologous in H^{2}(A, \widehat{A})$. 
The short exact sequence

$$
0 \rightarrow \widehat{A} \rightarrow \widehat{U(\mathcal{B})} \rightarrow \widehat{\operatorname{ker}\left(\pi_{1}\right)} \rightarrow 0
$$

induces a long exact sequence in cohomology

$$
\cdots \rightarrow \operatorname{Hom}\left(A, \widehat{\operatorname{ker}\left(\pi_{1}\right)}\right) \stackrel{d_{1}}{\rightarrow} H^{2}(A, \widehat{A}) \rightarrow H^{2}(A, \widehat{U(\mathcal{B})}) \rightarrow \cdots
$$

The set

$$
X_{\chi}:=\left\{\tilde{j}: A \rightarrow \operatorname{Aut}_{\otimes}^{\operatorname{ker}\left(\pi_{1}\right)}\left(\operatorname{Id}_{\mathcal{B}_{e}}\right)\left|\delta(\tilde{j})=\chi_{\lambda}\right|_{\mathcal{B}}\right\}
$$

is a torsor over the abelian group $\operatorname{Hom}\left(A, \widehat{\operatorname{ker}\left(\pi_{1}\right)}\right)$. It follows from Proposition 4.9 that for each $\tilde{j} \in X_{\chi}$ there is $j: A \rightarrow$ Aut $_{\otimes}\left(\operatorname{Id}_{\mathcal{B}}\right)$ such that $\tilde{j}_{a}=\left.\left(j_{a}\right)\right|_{\mathcal{B}_{e}}$ for each $a \in A$. Hence the natural isomorphisms

$$
\omega_{j}(a, b):=\chi_{\lambda(a, b)} \circ j_{a}^{-1} \circ j_{b}^{-1} \circ j_{a b} \in \operatorname{Aut}_{\otimes}^{A}\left(\operatorname{Id}_{\mathcal{B}}\right)
$$

define a 2-cocycle $\omega_{j} \in Z^{2}(A, \widehat{A})$, and again by Proposition 4.9 the cohomology class of $\omega_{j}$ only depend on $\tilde{j}$, and we will denote by

$$
\omega_{\tilde{j}} \in H^{2}(A, \widehat{A}) \text {. }
$$

Note that if $j^{\prime}, j^{\prime \prime} \in X_{\chi}$, then $\omega_{\tilde{j} / \tilde{j}^{\prime \prime}}=d_{1}\left(\tilde{j} / \tilde{j}^{\prime \prime}\right)$, where $d_{1}$ is defined in (4.19).

As a result of the Lemmas 4.12, 4.14 and the previous discussion, we obtain the following result.

Theorem 4.15. Let $\lambda$ be an associative zesting. Then there is a braided zesting associated if and only if the cohomology classes of $O_{1}(\lambda)(a,-,-) \in H^{2}\left(A, \mathbb{k}^{\times}\right)$vanish for all $a \in A$, and there exist $\tilde{j} \in X_{\lambda}$ such that $O_{2}(\lambda)=\omega_{\tilde{j}} \in H^{2}(A, \widehat{A})$.

Corollary 4.16. Let $\lambda$ be an associative zesting such that $\left.\chi_{\lambda(a, b)}\right|_{\mathcal{B}_{e}}=\operatorname{Id}_{\mathcal{B}_{e}}$ for all $a, b \in A$. Then there is a braided zesting of the form (Id, $t$ ) if and only if the cohomology classes of $O_{1}(\lambda)(a,-,-) \in H^{2}\left(A, \mathbb{k}^{\times}\right)$vanish for all $a \in A$, and $O_{2}(\lambda)=\left[\chi_{\chi}\right] \in H^{2}(A, \widehat{A})$.

\section{Twist Zesting And its Modular Data}

Given a premodular tensor category $\mathcal{B}$ and a braided zesting $\left(\mathcal{B}^{\lambda}, t\right)$, we would like to provide this zested category with a ribbon structure. In a customary abuse of notation we denote by $\theta_{X}$ both the automorphism in $\operatorname{Aut}_{\mathcal{B}}(X)$ and the scalar by which it acts when $X$ is a simple object. Similarly, we will denote the scalar by which $\chi_{a}$ acts on a simple object $X \in \mathcal{B}$ by $\chi_{a}(X)$ as well.

Proposition 5.1. Let $A$ be a finite abelian group, $\mathcal{B}$ be a faithfully $A$-graded braided tensor category with twist $\theta$ and $(\lambda, j, t)$ a braided zesting. We will denote by

$$
t^{(2)}: A \times A \rightarrow \mathbb{k}^{\times}
$$


the symmetric function defined by $t(b, a) \circ t(a, b)=: t^{(2)}(a, b) \operatorname{id}_{\lambda(a, b)}$ for all $a, b \in A$. Let $f: A \rightarrow \mathbb{K}^{\times}$be a function and consider the natural isomorphism

$$
\theta_{X_{a}}^{f}:=f(a) \theta_{X_{a}}, \quad a \in A, X_{a} \in \mathcal{B}_{a},
$$

then

(i) $\theta^{f}$ is a twist if and only if

$$
f(a+b) \chi_{\lambda(a, b)}\left(X_{a}\right) \chi_{\lambda(a, b)}\left(Y_{b}\right) \theta_{\lambda(a, b)}=f(a) f(b) j_{a}\left(Y_{b}\right) j_{b}\left(X_{a}\right) t^{(2)}(a, b), \quad f(0)=1,
$$

for all $a, b \in A, X_{a} \in \mathcal{B}_{a}, Y_{b} \in \mathcal{B}_{b}$.

(ii) If $\theta$ is a ribbon twist for $\mathcal{B}$ then $\theta^{f}$ is a ribbon twist if additionally

$$
f(a)=f(-a) \chi_{\lambda(a,-a)}\left(X_{a}\right) \theta_{\lambda(a,-a)}
$$

for all $a \in A, X_{a} \in \mathcal{B}_{a}$.

(iii) If $f^{\prime}, f: A \rightarrow \mathbb{k}^{\times}$is a pair of functions satisfying (5.1), then $f / f^{\prime}: A \rightarrow \mathbb{k}^{\times}$ is a character. Moreover, the set of all functions satisfying (5.1) is a torsor over $\widehat{A}$ and the set of all functions satisfying (5.1) and (5.2) is a torsor over $\widehat{A / 2 A}$.

Proof. Let $X_{a} \in \mathcal{B}_{a}$ and $Y_{b} \in \mathcal{B}_{b}$ simple objects. Then we have that

$$
\left(\theta_{X_{a}}^{f} \stackrel{\lambda}{\otimes} \theta_{Y_{b}}^{f}\right) \circ c_{Y_{a}, X_{a}}^{\lambda} \circ c_{X_{a}, Y_{b}}^{\lambda}=f(a) f(b) j_{a}\left(Y_{b}\right) j_{b}\left(X_{a}\right) t^{(2)}(a, b) \theta_{X_{a} \otimes Y_{b}} \otimes \operatorname{id}_{\lambda(a, b)},
$$

and

$$
\theta_{X_{a} \otimes Y_{b}}^{f}=f(a+b) \chi_{\lambda(a, b)}\left(X_{a}\right) \chi_{\lambda(a, b)}\left(Y_{b}\right) \theta_{\lambda(a, b)} \theta_{X_{a} \otimes Y_{b}} \operatorname{id}_{\lambda(a, b)} .
$$

Hence, (5.1) holds if and only if

$$
\left(\theta_{X_{a}}^{f} \stackrel{\lambda}{\otimes} \theta_{Y_{b}}^{f}\right) \circ c_{Y_{a}, X_{a}}^{\lambda} \circ c_{X_{a}, Y_{b}}^{\lambda}=\theta_{X_{a} \otimes Y_{b}}^{f},
$$

that is, if $\theta^{f}$ is a twist.

For the ribbon condition, we have that

$$
\begin{aligned}
\theta_{\bar{X}_{a}}^{f} & =f(-a) \chi_{\lambda(a,-a)}\left(X_{a}\right) \theta_{X_{a}^{*}} \theta_{\lambda(a,-a)^{*}} \operatorname{id}_{X^{*} a \otimes \lambda(a,-a)^{*}} \\
& =f(-a) \chi_{\lambda(a,-a)}\left(X_{a}\right) \theta_{X_{a}} \theta_{\lambda(a,-a)} \operatorname{id}_{\bar{X}_{a}}
\end{aligned}
$$

and

$$
\theta_{X_{a}}^{f}=f(a) \theta_{X_{a}} \operatorname{id}_{X_{a}}
$$

for all simple objects $X_{a} \in \mathcal{B}_{a}$. Hence $\left(\theta_{X_{a}}^{f}\right)^{*}=\theta_{\bar{X}_{a}}^{f}$ if and only if (5.2) holds.

For (iii), let $f$ and $f^{\prime}$ both satisfy the conditions in (5.1) and set $\eta(a)=f(a) / f^{\prime}(a)$. Since $f(a) f(b) / f(a+b)=f^{\prime}(a) f^{\prime}(b) / f^{\prime}(a+b)$ we find that $\eta(a+b)=\eta(a) \eta(b)$. A similar argument implies that if $f, f^{\prime}$ satisfy the condition (5.2) then $\eta(a)=\eta(-a)$, so $\eta(2 a)=1$, that is $\eta \in \widehat{A / 2 A}$. 
Remark 5.2. In practice, the ribbon zestings are easy to find. The symmetric function

$$
\begin{aligned}
s: A \times A & \rightarrow \mathbb{k}^{\times} \\
(a, b) & \mapsto \frac{\chi_{\lambda(a, b)}\left(X_{a}\right) \chi_{\lambda(a, b)}\left(Y_{b}\right) \theta_{\lambda(a, b)}}{j_{a}\left(Y_{b}\right) j_{b}\left(X_{a}\right) t^{(2)}(a, b)},
\end{aligned}
$$

should be independent of the choice of $X_{a} \in \mathcal{B}_{a}, Y_{b} \in \mathcal{B}_{b}$. If the function $s$ is a 2-cocycle, (an easy condition to check) since $s$ symmetric we can find a function $f$ satisfying condition (5.1).

When zesting with respect to the universal grading, the scalars $\chi_{\lambda(i, j)}\left(X_{k}\right)$ only depend on the graded component of the simple object $X_{k} \in \mathcal{B}_{k}$, so we may denote it $\chi_{\lambda(i, j)}(k)$, and take $j_{a}=$ id. In this case the conditions (5.1) and (5.2) for twist zesting reduce to a simpler form:

Corollary 5.3. Suppose $\mathcal{B}$ is a braided fusion category with a twist and $\left(\mathcal{B}^{\lambda}, t\right)$ is a braided A-zesting where $A=U(\mathcal{B})$ is the universal grading group. Then for $\theta^{f} \in$ Aut $\left(\operatorname{Id}_{\mathcal{B}}\right)$ defined in Proposition 5 we have:

(a) if $f(a+b) \chi_{\lambda(a, b)}(a+b) \theta_{\lambda(a, b)}=f(a) f(b) t^{(2)}(a, b)$,

then $\theta^{f}$ defines a twist on $\left(\mathcal{B}^{\lambda}, t\right)$ and

(b) if $\theta$ is a ribbon twist on $\mathcal{B}$ and $f(a)=\chi_{\lambda(a,-a)}(a) \theta_{\lambda(a,-a)} f(-a)$ then $\theta^{f}$ defines a ribbon twist on $\left(\mathcal{B}^{\lambda}, t\right)$.

\subsection{Modular data of a ribbon zesting.}

Definition 5.4. A quadruple $(\lambda, j, t, f)$ where $(\lambda, j, t)$ is a braided zesting and $f$ : $A \rightarrow \mathbb{k}^{\times}$is a function satisfying equations (5.1) and (5.2) is called a ribbon zesting.

Proposition 5.5. Let $(\lambda, j, t, f)$ be a a ribbon zesting, then quantum trace of an endomorphism of $s: X_{a} \rightarrow X_{a}$ in $\mathcal{B}^{(\lambda, f)}$, for $a \in A, X_{a} \in \mathcal{B}_{a}$ is

$$
\operatorname{Tr}^{f}(s)=\frac{f(a)}{\operatorname{dim}(\lambda(-a, a)) t(a, a)} \operatorname{Tr}\left(j_{a}^{-1}\left(X_{a}\right) \circ s\right) .
$$

Proof. In this proof, without loss of generality, we will assume that $\mathcal{B}$ is a strict pivotal category, which means that the natural isomorphism between an object and its double dual is the identity morphism and also the pivotal structure is trivial.

The trace in $\mathcal{B}^{(\lambda, f)}$ is given by the formula

$$
\operatorname{Tr}^{f}(s)=\frac{1}{\operatorname{dim}(\lambda(-a, a))} \rho_{X_{a}^{*}} \circ(\tilde{\psi} \tilde{\otimes} \mathrm{id}) \circ(s \tilde{\otimes} \mathrm{id}) \circ \phi_{X_{a}},
$$

where $\tilde{\psi}$ is the pivotal structure in $\mathcal{B}^{(\lambda, f)}$ and $\rho$ and $\phi$ denote the evaluation and coevaluation maps in $\mathcal{B}^{(\lambda, f)}$ described in Subsection 3.3. So, in order to compute $\operatorname{Tr}^{f}(s)$, we need to compute the pivotal structure $\tilde{\psi}$ first. To do this, we can consider the Drinfeld isomporhism $\tilde{u}$ in $\mathcal{B}^{(\lambda, f)}$ which is related to the twist and pivotal structure by $\tilde{\psi}=\tilde{u} \circ \tilde{\theta}^{f}$.

The general formula of the Drinfeld isomorphism $\tilde{u}$ is given by

$$
\tilde{u}=\frac{1}{\operatorname{dim}(\lambda(-a, a))}\left(\rho_{X_{a}} \tilde{\otimes} \operatorname{id}_{X_{a}}\right) \circ\left(\operatorname{id}_{X_{a}^{*}} \tilde{\otimes} \tilde{c}_{X_{a}, X_{a}}^{-1}\right) \circ\left(\phi_{X_{a}^{*}} \tilde{\otimes} \operatorname{id}_{X_{a}}\right) .
$$


Applying this formula in our case, and assuming strict pivotality of $\mathcal{B}$, we get that $\tilde{u}$ can be written in terms of the data of the original category $\mathcal{B}$ and the zesting structure as

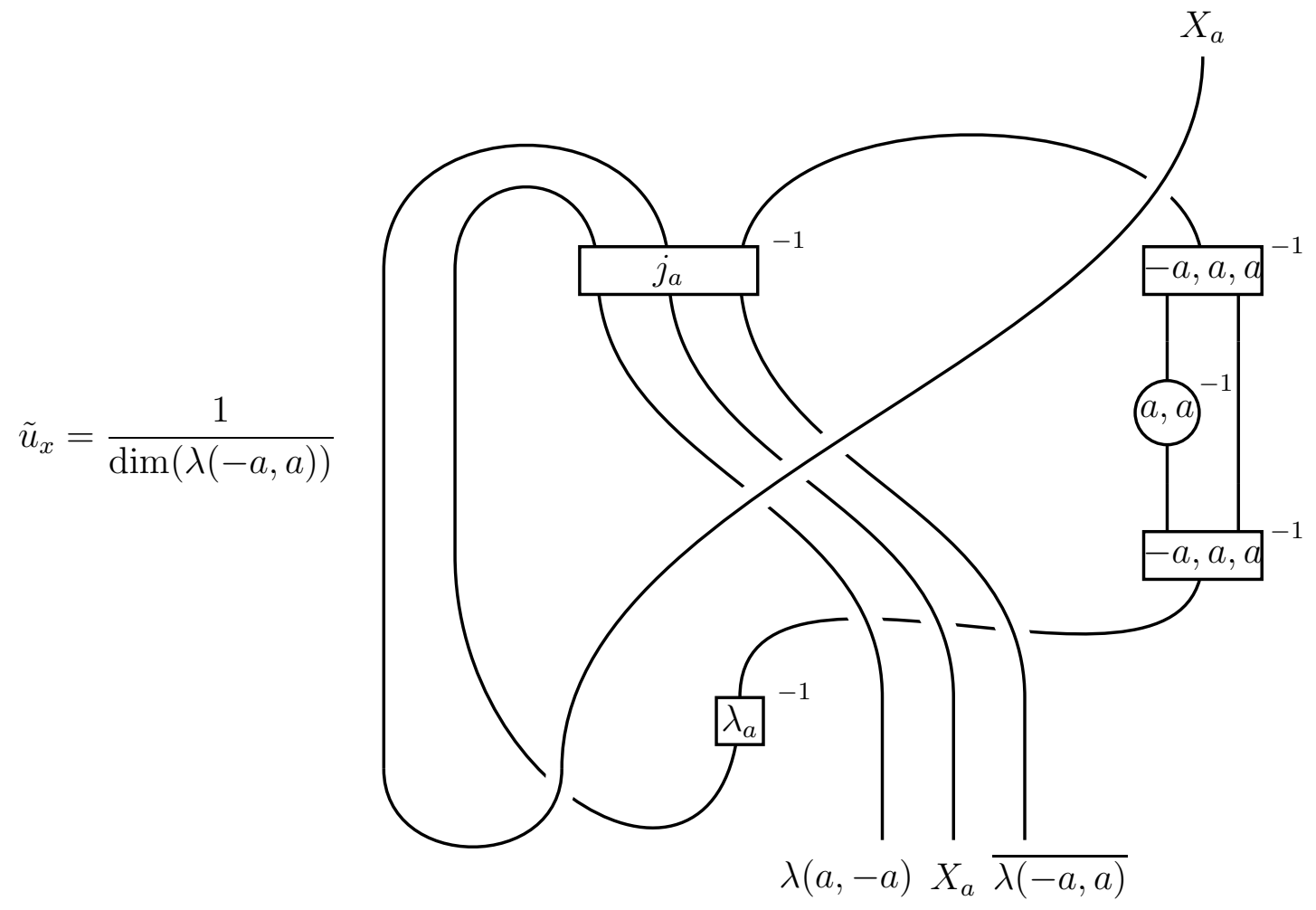

Applying the standard yoga of graphical calculus and using that $j_{g}$ is a tensor autoequivalence and $\mathcal{B}$ being strict pivotal, i.e. $u=\theta^{-1}$, we get the following expression 
for the Drinfeld isomorphism of $\mathcal{B}^{(\lambda, f)}$.

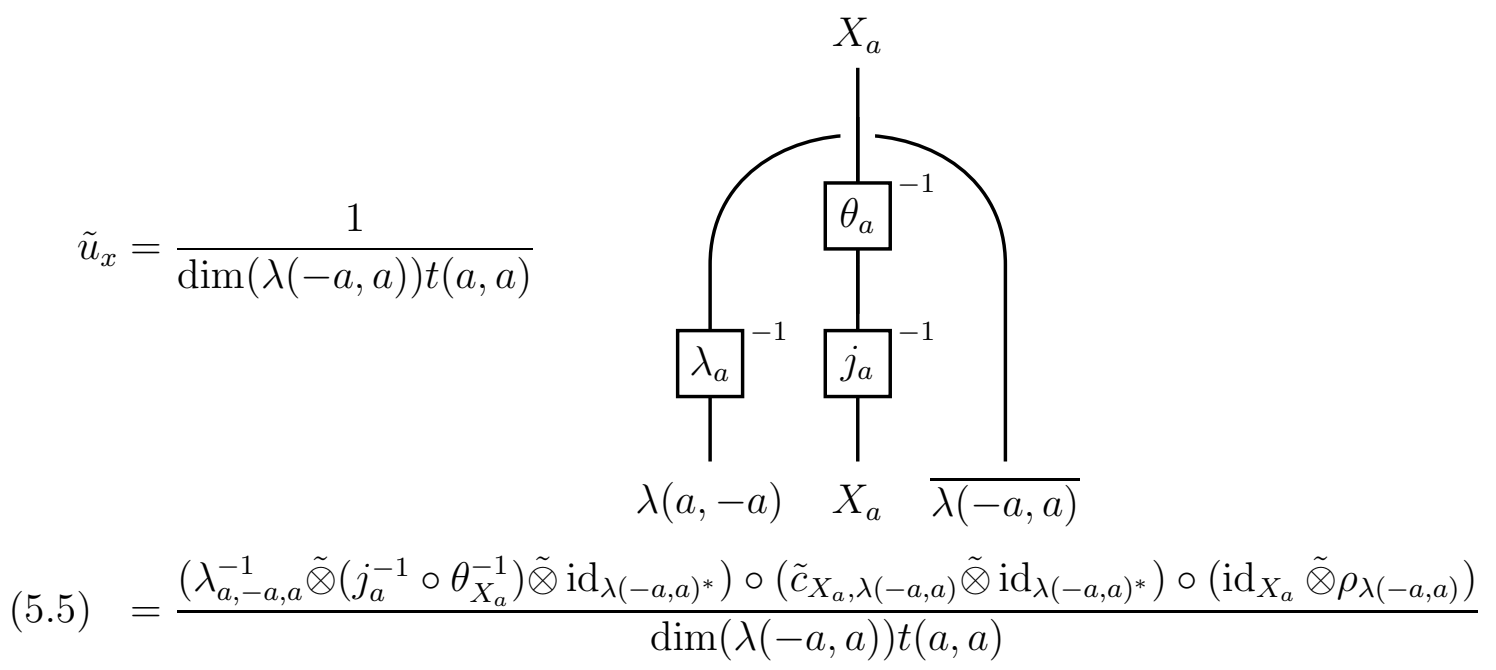

Notice that here, if we weren't assuming $\mathcal{B}$ is strict pivotal, we would get the Drinfeld isomorphism $u$ of $\mathcal{B}$ instead of $\theta^{-1}$ in Equality (5.4).

From this, assuming strict pivotality of $\mathcal{B}$ we get that the pivotal structure $\tilde{\psi}$ in $\mathcal{B}^{(\lambda, f)}$ is

$$
\begin{aligned}
\tilde{\psi} & =\frac{f(a)}{\operatorname{dim}(\lambda(-a, a)) t(a, a)}{ }=\frac{f(a)}{\operatorname{dim}(\lambda(-a, a)) t(a, a)}\left(\lambda_{a,-a, a}^{-1} \tilde{\otimes} j_{a}^{-1} \tilde{\otimes} \operatorname{id}_{\lambda(-a, a)^{*}}\right) \circ\left(\tilde{c}_{X_{a}, \lambda(-a, a)} \tilde{\otimes} \operatorname{id}_{\lambda(-a, a)^{*}}\right) \circ\left(\operatorname{id}_{X_{a}} \tilde{\otimes} \rho_{\lambda(-a, a)}\right)
\end{aligned}
$$

In this way, we get that the trace $\operatorname{Tr}^{f}$ in $\mathcal{B}^{(\lambda, f)}$ of a morphism $s \in \operatorname{Hom}\left(X_{a}, X_{a}\right)$ can be expressed in the graphical calculus by 


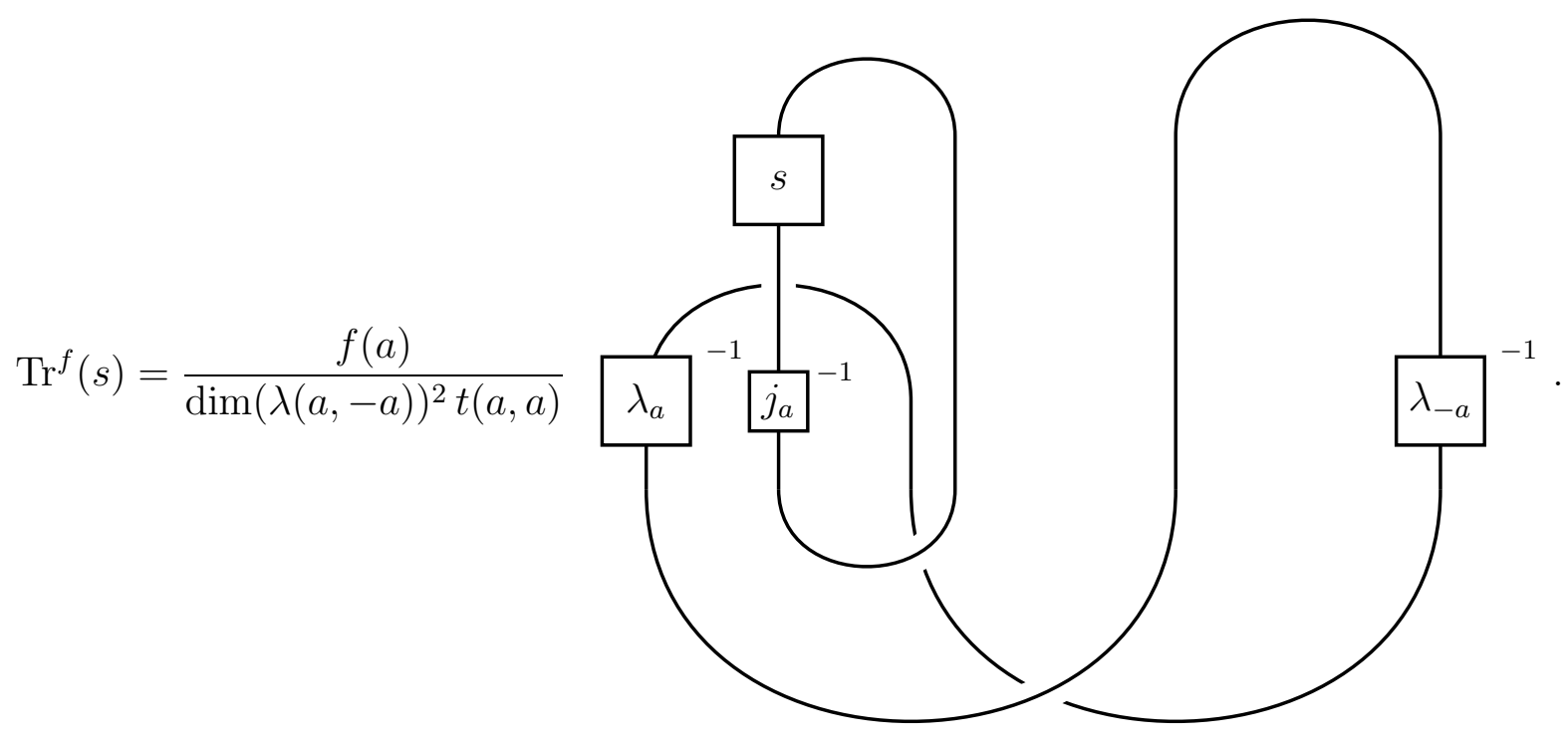

After expressing the closed loop involving morphisms on $X_{a}$ in terms of the trace in $\mathcal{B}$, we get

$$
\operatorname{Tr}^{f}(s)=\frac{f(a)}{\operatorname{dim}(\lambda(a,-a))^{2} t(a, a)} \operatorname{Tr}\left(j_{a}^{-1}\left(X_{a}\right) \circ s\right)
$$

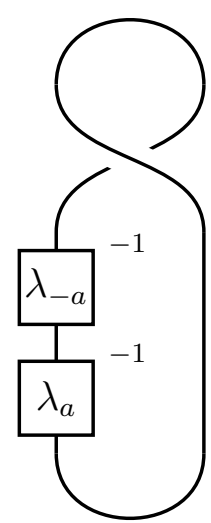


Finally, we apply the associative zesting condition of Figure 2 with $1=3=a$ and $2=4=-a$ and standard graphical calculus to get the equation

$$
\begin{aligned}
\operatorname{Tr}^{f}(s) & =\frac{f(a)}{\operatorname{dim}(\lambda(a,-a))^{2} t(a, a)} \operatorname{Tr}\left(j_{a}^{-1}\left(X_{a}\right) \circ s\right) \\
& =\frac{f(a)}{\operatorname{dim}(\lambda(a,-a)) t(a, a)} \operatorname{Tr}\left(j_{a}^{-1}\left(X_{a}\right) \circ s\right)
\end{aligned}
$$

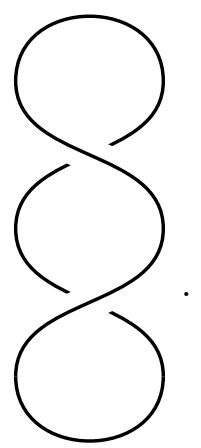

Lemma 5.6. Let $\lambda=(\lambda, j, t, f)$ be a a ribbon zesting. Then

$$
\begin{aligned}
\operatorname{Tr}^{f}\left(c_{Y_{b}, X_{a}}^{\lambda} \circ c_{X_{a}, Y_{b}}^{\lambda}\right)= & \frac{\operatorname{dim}(\lambda(a+b,-a-b)) \operatorname{dim}(\lambda(a, b)) t^{(2)}(a, b) f(a+b)}{t(a+b, a+b)} \\
& \times m\left(j, X_{A}, Y_{b}\right) \operatorname{Tr}\left(c_{Y^{*}, X} \circ c_{X, Y^{*}}\right)
\end{aligned}
$$

where

$$
m\left(j, X_{a}, Y_{b}\right)=\frac{j_{a}\left(Y_{b}\right) j_{b}\left(X_{a}\right)}{j_{a b}\left(X_{a}\right) j_{a b}\left(Y_{b}\right) j_{a b}(\lambda(a, b))},
$$

for all $a, b \in A, X_{a} \in \operatorname{Irr}\left(\mathcal{B}_{a}\right), Y_{b} \in \operatorname{Irr}\left(\mathcal{B}_{b}\right)$.

Proof. Let $X_{a} \in \operatorname{Irr}\left(\mathcal{B}_{a}\right), Y_{b} \in \operatorname{Irr}\left(\mathcal{B}_{b}\right)$, where $a, b \in A$. We have that

$$
j_{a b}^{-1} \circ c_{Y_{b}, X_{a}}^{\lambda} \circ c_{X_{a}, Y_{b}}^{\lambda}=m\left(j, X_{a}, Y_{b}\right) t^{(2)}(a, b)\left(c_{Y_{b}, X_{a}} \circ c_{X_{a}, Y_{b}}\right) \otimes \operatorname{id}_{\lambda(a, b)}
$$

for all pairs of simple objects $X_{a}, Y_{b}$.

Then using Proposition 5.5 we have

$$
\begin{aligned}
\operatorname{Tr}^{f}\left(c_{Y_{b}, X_{a}}^{\lambda} \circ c_{X_{a}, Y_{b}}^{\lambda}\right)= & \frac{\operatorname{dim}(\lambda(a+b,-a-b)) f(a+b)}{t(a+b, a+b)} m\left(j, X_{A}, Y_{b}\right) \\
& \times t^{(2)}(a, b) \operatorname{Tr}\left(\left(c_{Y_{a}, X_{a}} \circ c_{X_{a}, Y_{a}}\right) \otimes \operatorname{id}_{\lambda(a, b)}\right) \\
= & \frac{\lambda(a+a,-a-a) \operatorname{dim}(\lambda(a, b)) f(a+b)}{t(a+b, a+b)} m\left(j, X_{A}, Y_{b}\right) \\
& \times t^{(2)}(a, b) \operatorname{Tr}^{f}\left(c_{Y_{a}, X_{a}}^{\lambda} \circ c_{X_{a}, Y_{a}}\right) .
\end{aligned}
$$

Recall that the modular data of a premodular category $\mathcal{B}$ is the following pair of matrices indexed over $\operatorname{Irr}(\mathcal{B})$ :

(i) S-matrix. $S_{X, Y}=\operatorname{Tr}\left(c_{Y^{*}, X} \circ c_{X, Y^{*}}\right)$, 
(ii) T-matrix. $T_{X, Y}=\theta_{X} \delta_{X, Y}$.

Theorem 5.7. Let $\lambda=(\lambda, j, t, f)$ be a a ribbon zesting. The T-matrix and $S$-matrix of $\mathcal{B}^{(\lambda, f)}$ are given by the following formulas

$$
\begin{aligned}
T_{X_{a}, X_{a}}^{\lambda} & =f(a) T_{X_{a}, X_{a}} \\
S_{X_{a}, Y_{b}}^{\lambda} & =\frac{\operatorname{dim}(\lambda(a-b,-a+b)) \operatorname{dim}(\lambda(a,-b)) t^{(2)}(a,-b) f(a-b)}{t(a-b, a-b)} \\
& \times m\left(j, X_{a}, Y_{b}^{*} \otimes \lambda(b,-b)^{*}\right) \operatorname{dim}\left(X_{a}\right)^{-1} S_{X_{a}, Y_{b}} S_{X_{a}, \lambda(b,-b)}
\end{aligned}
$$

for all $a, b \in A, X_{a} \in \mathcal{B}_{a}, Y_{b} \in \mathcal{B}_{b}$.

Proof. The formula for $T^{\lambda}$ is a direct consequence of the definition of $\theta^{f}$.

For the $S$-matrix of $\mathcal{B}^{(\lambda, f)}$ we use Lemma 5.6 and the fact that $\bar{X}_{a}=X_{a}^{*} \otimes \lambda(a,-a)^{*}$

$$
\begin{aligned}
S_{X_{a}, Y_{b}}^{\lambda} & =\frac{\operatorname{dim}(\lambda(a-b,-a+b)) \operatorname{dim}(\lambda(a,-b)) t^{(2)}(a,-b) f(a-b)}{t(a-b, a-b)} \\
& \times m\left(j, X_{a}, Y_{b}^{*} \otimes \lambda(b,-b)^{*}\right) S_{X_{a}, \lambda(b,-b) \otimes Y_{b}} \\
= & \frac{\operatorname{dim}(\lambda(a-b,-a+b)) \operatorname{dim}(\lambda(a,-b)) t^{(2)}(a,-b) f(a-b)}{t(a-b, a-b)} m\left(j, X_{a}, Y_{b}^{*} \otimes \lambda(b,-b)^{*}\right) \\
& \times \operatorname{dim}\left(X_{a}\right)^{-1} S_{X_{a}, Y_{b}} S_{X_{a}, \lambda(b,-b)},
\end{aligned}
$$

where we used in the last equality that $\operatorname{dim}(X) S_{X, Y \otimes a}=S_{X, Y} S_{X, a}$ for every invertible object, see [14, Proposition 8.13.10].

5.2. Müger center. We want to describe the Müger center $\mathcal{Z}_{2}\left(\mathcal{B}^{(\lambda, t)}\right)=\left\{X_{a} \in\right.$ $\left.\mathcal{B}_{a} \mid \tilde{c}_{X_{a}, Y_{b}}^{2}=\operatorname{id}_{X_{a} \tilde{\otimes} Y_{b}} \forall Y_{b} \in \mathcal{B}_{b}, b \in A\right\}$ of the zesting $\mathcal{B}^{(\lambda, t)}$ of $\mathcal{B}$. We have that

$$
\mathcal{Z}_{2}\left(\mathcal{B}^{(\lambda, t)}\right)=\left\{X_{a} \in \mathcal{B}_{a} \mid c_{X_{a}, Y_{b}}^{2}=j_{a}\left(Y_{b}\right)^{-1} j_{b}\left(X_{a}\right)^{-1} t^{-2}(a, b) \operatorname{id}_{X_{a} \otimes Y_{b}}, \forall Y_{b} \in \mathcal{B}_{b}, b \in A\right\} .
$$

This means that $X_{a} \in \mathcal{C}_{a}$ is in the Müger center of $\mathcal{B}^{(\lambda, t)}$ if $X_{a}$ projectively centralizes $Y_{b}$ (and the corresponding scalar is $j_{a}\left(Y_{b}\right)^{-1} j_{b}\left(X_{a}\right)^{-1} t^{-2}(a, b)$ ), for all $Y_{b} \in \mathcal{B}_{b}, b \in A$. Recall from [14] that for $\mathcal{D} \subset \mathcal{C}$ the centralizer of $\mathcal{D}$ in $\mathcal{C}$ is denoted $C_{\mathcal{C}}(\mathcal{D})$.

Lemma 5.8. Assume that $j$ is trivial. Consider a premodular A-graded fusion category $\mathcal{B}$ and a braided zesting $\mathcal{B}^{(\lambda, t)}$. If $C_{\mathcal{B}}\left(\mathcal{B}_{a d}\right) \subseteq \mathcal{B}_{p t}$ then the Müger center of the zested category

$$
\mathcal{Z}_{2}\left(\mathcal{B}^{(\lambda, t)}\right)=\left\{X_{a} \in \mathcal{B}_{a} \cap \mathcal{B}_{p t} \mid t^{2}(a, b)=\frac{\theta_{X_{a}} \theta_{Y_{b}}}{\theta_{X_{a} \otimes Y_{b}}}, \forall Y_{b} \in \mathcal{B}_{b}, b \in A\right\} .
$$

Proof. Recall that $t$ is trivial when one of the inputs lives in the trivial component of the $A$-grading. Now, since $j$ is trivial, if $X_{a} \in \mathcal{Z}_{2}\left(\mathcal{B}^{(\lambda, t)}\right)$ then $c_{X_{a}, Y_{0}}^{2}=\mathrm{id}_{X_{a} \otimes Y_{0}}$ for all $Y_{0} \in \mathcal{B}_{0}$. This means that $X_{a} \in C_{\mathcal{B}}\left(\mathcal{B}_{0}\right) \subseteq C_{\mathcal{B}}\left(\mathcal{B}_{a d}\right) \subseteq \mathcal{B}_{p t}$. Therefore $\mathcal{Z}_{2}\left(\mathcal{B}^{(\lambda, t)}\right) \subseteq \mathcal{B}_{p t}$. 
Moreover, since $X_{a} \in \mathcal{Z}_{2}\left(\mathcal{B}^{(\lambda, t)}\right) \subseteq \mathcal{B}_{p t}$, we have that $\chi_{X_{a}}\left(Y_{b}\right)=t^{-2}(a, b)$. From the fact that $\chi_{X_{a}}\left(Y_{b}\right)=\frac{\theta_{X_{a} \otimes Y_{b}}}{\theta_{X_{a}} \theta_{Y_{b}}}$, we get the desired characterization of the Müger center $\mathcal{Z}_{2}\left(\mathcal{B}^{(\lambda, t)}\right)$ of the zested category.

Remark 5.9. Notice that if $\mathcal{B}$ is modular or super-modular (i.e. $\mathcal{Z}_{2}(\mathcal{B})=$ Vec or sVec), we have that $\mathcal{Z}_{2}\left(\mathcal{B}^{(\lambda, t)}\right) \subseteq C_{\mathcal{B}}\left(\mathcal{B}_{a d}\right) \subseteq \mathcal{B}_{p t}$. If $\mathcal{B}$ is pointed the condition is trivially satisfied too.

The following example shows that zesting a non-degenerate category may yield a degenerate one, and conversely, so that Müger centers are not zesting invariant.

Example 5.10. The pointed modular category $\mathcal{C}(\mathbb{Z} / 3, Q)$ with quadratic form $Q(a)=$ $e^{2 a^{2} \pi i / 3}=\theta_{a}$ is naturally $\mathbb{Z} / 3$-graded. If we take the trivial associative $\mathbb{Z} / 3$-zesting (i.e. trivial 2- and 3-cocycles $\lambda(a, b)=\mathbf{1}$ and $\lambda(a, b, c)=1)$ then the braided zestings correspond to a choice of a bicharacter $t: \mathbb{Z} / 3 \times \mathbb{Z} / 3 \rightarrow \mathbb{k}^{\times}$. We can take $t(a, b)=e^{2 \pi i a b / 3}$ so that $t^{2}(a, b)=Q(a) Q(b) / Q(a+b)=e^{-2 \pi i a b / 3}$, which implies $\mathcal{C}(\mathbb{Z} / 3, Q)^{\lambda, t}$ is symmetric.

The converse is also possible: for the symmetric pointed category $\mathcal{C}(\mathbb{Z} / 3, P)$ with $P(a)=1$ again take the trivial associative zesting. Now a non-trivial braided zesting corresponds to a non-trivial bicharacter $t$, which yields a non-degenerate braiding.

On the other hand, if we zest with respect to a group $A$ that generates a symmetric pointed subcategory and $A$ does not contain any transparent objects then the Müger center does not change:

Proposition 5.11. Let $\mathcal{B}$ a braided fusion category and $A \subset \operatorname{Inv}(\mathcal{B})$ a subgroup such that $\chi_{a} \neq \mathrm{id}$ for all $a \in A-\{0\}$. Consider the $\widehat{A}$-grading

$$
\mathcal{B}_{\gamma}=\left\{X \in \mathcal{B}: \chi_{a}(X)=\gamma(a) \operatorname{id}_{X}, \quad \forall a \in A\right\}, \quad \gamma \in \widehat{A} .
$$

For any $\widehat{A}$-braided zesting $(\lambda, j, t)$, such that

(i) the category generated by $A$ is symmetric,

(ii) $j_{\gamma}=$ id for all $\gamma \in \widehat{A}$,

the Müger center of $\mathcal{B}$ and $\mathcal{B}^{(\lambda, t)}$ coincide. In particular, $\mathcal{B}$ is non-degenerate if and only if $\mathcal{B}^{(\lambda, t)}$ is non-degenerate.

Proof. Since $\mathcal{B}_{1}=\left\{X: c_{X, a} \circ c_{a, X}=\mathrm{id}_{a \otimes X}, \forall a \in A\right\}$, we have that $\mathcal{Z}_{2}(\mathcal{B}) \subset \mathcal{B}_{1}$. Hence, if $X \in \mathcal{Z}_{2}(\mathcal{B})$ and $Y_{\gamma} \in \mathcal{B}_{\gamma}$, then $X \stackrel{\lambda}{\otimes} Y_{\gamma}=X \otimes Y_{\gamma}$ and $c_{X, Y_{\gamma}}^{\lambda}=c_{X, Y_{\gamma}}$. Hence $\mathcal{Z}_{2}(\mathcal{B}) \subset \mathcal{Z}_{2}\left(\mathcal{B}^{(\lambda, t)}\right)$

Conversely, since the category generated by $A$ is symmetric, we have that $a \in \mathcal{B}_{1}$ for all $a \in A$. If

$$
X_{\gamma} \in \mathcal{Z}_{2}\left(\mathcal{B}^{(\lambda, t)}\right) \cap \mathcal{B}_{\gamma}, \quad \gamma \in \widehat{A}
$$

then

$$
\operatorname{id}_{X_{\gamma} \otimes a}=c_{a, X_{\gamma}}^{\lambda} \circ c_{X_{\gamma}, a}^{\lambda}=c_{a, X_{\gamma}} \circ c_{X_{\gamma}, a}, \quad \forall a \in A
$$


hence $\gamma=1$. Then,

$$
c_{X, Y}^{\gamma}=c_{X, Y}, \quad c_{Y, X}^{\lambda}=c_{Y, X}
$$

for all $X \in \mathcal{Z}_{2}\left(\mathcal{B}^{(\lambda, t)}\right)$ and $Y \in \mathcal{B}$. This implies that $\mathcal{Z}_{2}\left(\mathcal{B}^{(\lambda, t)}\right) \subset \mathcal{Z}_{2}(\mathcal{B})$, and then $\mathcal{Z}_{2}\left(\mathcal{B}^{(\lambda, t)}\right)=\mathcal{Z}_{2}(\mathcal{B})$

Corollary 5.12. Let $\mathcal{B}$ be a non-degenerate braided fusion category such that $\mathcal{B}_{p t}$ is symmetric. Then any braided $U(\mathcal{B})$-zesting of $\mathcal{B}$ is non-degenerate.

Remark 5.13. The pointed subcategory $\mathcal{B}_{p t}$ is symmetric for any non-degenerate braided fusion category with $\mathcal{B}_{p t} \subseteq \mathcal{B}_{a d}$.

5.3. Braid group image. Let $\mathbb{B}_{n}$ denote the braid group on $n$ strands and $\sigma_{i}$ its generators. Given an object $X$ in a braided fusion category $\mathcal{B}$ we will denote the associated braid group representation by

$$
\begin{aligned}
\rho_{n}^{X}: \mathbb{B}_{n} & \rightarrow \operatorname{Aut}_{\mathcal{B}}\left(X^{\otimes n}\right) \\
\sigma_{i} & \mapsto \boldsymbol{a}^{-1} \circ\left(\operatorname{id}_{X}^{\otimes i-1} \otimes c_{X, X} \otimes \operatorname{id}_{X}^{\otimes n-i-1}\right) \circ \boldsymbol{a},
\end{aligned}
$$

where $\boldsymbol{a}$ denotes the appropriate composition of associativity constraints in $\mathcal{B}$. Note that the fact that this morphism is group homomorphism follows from the hexagon axioms of $\mathcal{B}$ and Mac Lane's coherence theorem. The category $\mathcal{B}$ is said to have property $F$ if $\rho_{n}^{X}$ has finite image for all $n$ and all objects $X[23]$.

Consider a braided fusion category $\mathcal{B}$ with an $A$-grading and $\lambda=(\lambda, j, t)$ a braided $A$-zesting. Here we study how the image of the braid group is modified under the zesting operation.

For any $X_{a} \in \mathcal{B}_{a}, Y_{b} \in \mathcal{B}_{b}, Z_{c} \in \mathcal{B}_{c}$ and $a, b, c \in A$, we have an algebra isomorphism

$$
\begin{aligned}
\psi_{X_{a}, Y_{b}, Z_{c}}: \operatorname{End}_{\mathcal{B}}\left(X_{a} \otimes Y_{b} \otimes Z_{c}\right) & \rightarrow \operatorname{End}_{\mathcal{B}^{\lambda}}\left(\left(X_{a} \stackrel{\lambda}{\otimes} Y_{b}\right) \stackrel{\lambda}{\otimes} Z_{c}\right) \\
f & \mapsto w^{-1}\left(f \otimes \operatorname{id}_{\lambda(a, b) \otimes \lambda(a+b, c)}\right) w
\end{aligned}
$$

where $w=\operatorname{id}_{X_{a} \otimes Y_{b}} \otimes c_{\lambda(a, b), Z_{c}} \otimes \operatorname{id}_{\lambda(a+b, c)}$. For $X_{a} \in \mathcal{B}_{a}^{\lambda}$ and $n \in \mathbb{Z}_{>0}$, we define inductively the algebra isomorphism

$$
\psi_{n}^{X_{a}}: \operatorname{End}_{\mathcal{B}}\left(X_{a}^{\otimes n}\right) \rightarrow \operatorname{End}_{\mathcal{B}^{\lambda}}\left(X_{a}^{\otimes n}\right)
$$


by $\psi_{n}^{X_{a}}=\left(\psi_{n-1}^{X_{a}} \stackrel{\lambda}{\otimes} \operatorname{id}_{X_{a}}\right) \circ \psi_{X_{a}^{\lambda}{ }^{\lambda}-2, X_{a}, X_{a}}$, where $X^{\stackrel{\lambda}{\otimes}}=X^{\stackrel{\lambda}{\otimes} n-1} \stackrel{\lambda}{\otimes} X$. Graphically, given for $f \in \operatorname{End}_{\mathcal{B}}\left(X_{a}^{\otimes n}\right)$, we have that $\psi_{n}^{X_{a}}(f)$ is given by

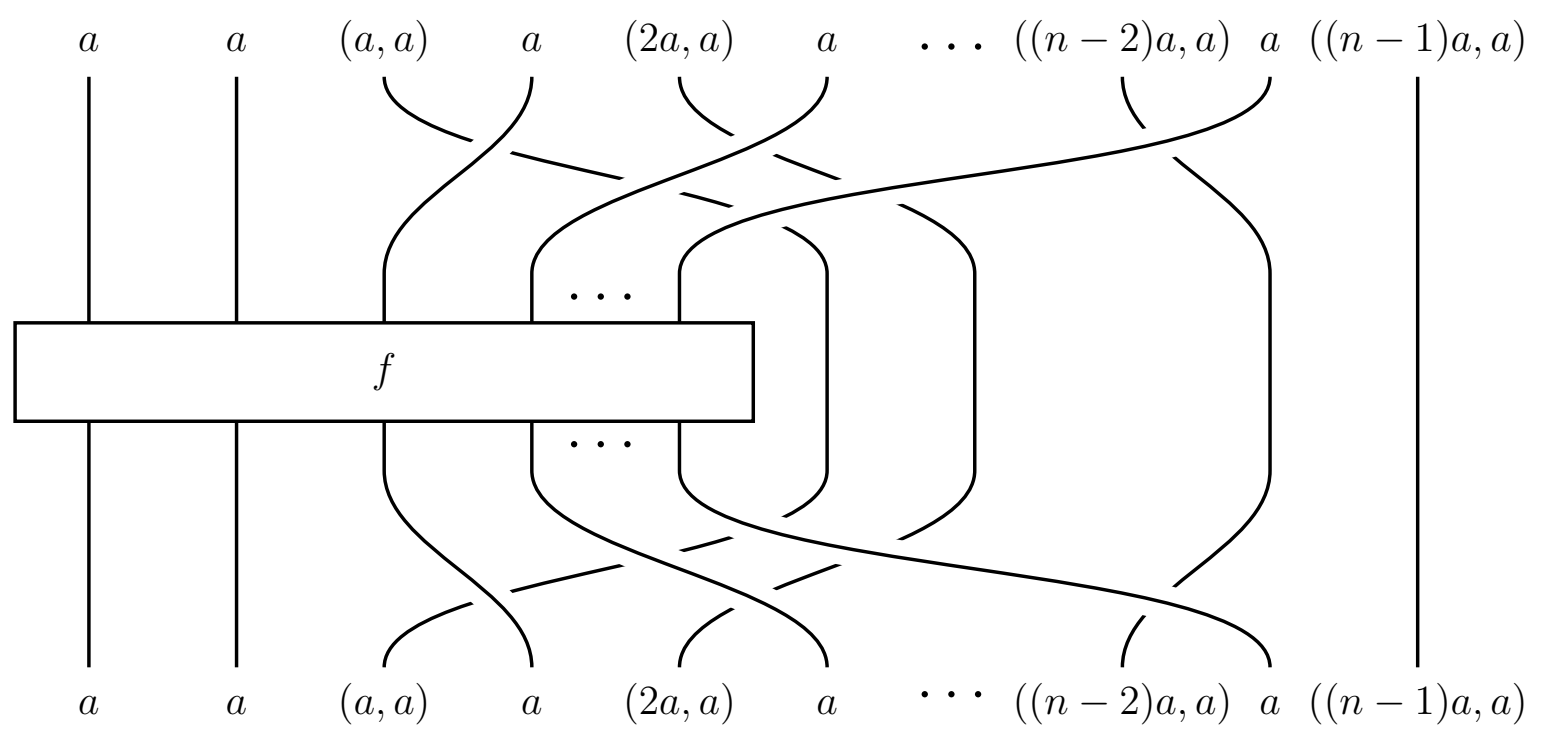

Theorem 5.14. Let $\mathcal{B}$ be a braided fusion category and $\lambda=(\lambda, j, t)$ a braided $A$ zesting. Then for all $n>0$ and $X_{a} \in \mathcal{B}_{a}$ simple homogeneous object, we have

$$
\rho_{n}^{\lambda}\left(\sigma_{i}\right)=j_{a}\left(X_{a}\right) t(a, a) \psi_{n}\left(\rho_{n}\left(\sigma_{i}\right)\right), \quad 1 \leq i<n,
$$

where $\rho^{\lambda}$ is the braid group representation associated to $\mathcal{B}^{\lambda}, \psi_{n}$ was defined in 5.14 , and $j_{a}\left(X_{a}\right), t(a, a) \in \mathbb{k}^{\times}$are the scalars associated with the braid $c_{X_{a}, X_{a}}^{\lambda}$.

Proof. If $X_{a} \in \mathcal{B}_{a}$ is a simple object, we have that $c_{X_{a}, X_{a}}^{\lambda}=j_{a}\left(X_{a}\right) t(a, a) c_{X_{a}, X_{a}} \otimes \mathrm{id}_{\lambda(a, a)}$. A simple graphical computation shows that

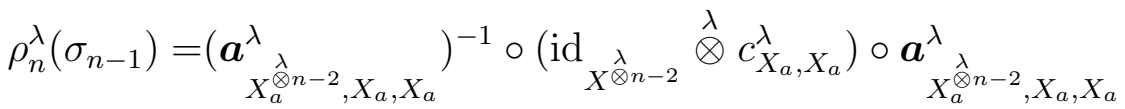

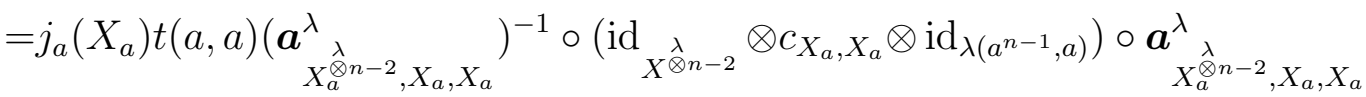

$$
\begin{aligned}
& =j_{a}\left(X_{a}\right) t(a, a) \psi_{n}\left(\rho_{n}\left(\sigma_{n-1}\right)\right) .
\end{aligned}
$$

For $i<n-1$, we have

$$
\begin{aligned}
& \rho_{n}^{\lambda}\left(\sigma_{i}\right)=\rho_{i+1}^{\lambda}\left(\sigma_{i}\right) \stackrel{\lambda}{\otimes} \mathrm{id} \underset{X^{\lambda(n-(i+1))}}{ } \\
& =j\left(X_{a}\right) t(a, a) \psi_{i+1}\left(\rho_{i+1}\left(\sigma_{i}\right)\right) \stackrel{\lambda}{\otimes} \mathrm{id}_{X^{\lambda}(n-1-i)} \\
& =j_{a}\left(X_{a}\right) t(a, a) \psi_{n}\left(\rho_{n}\left(\sigma_{i}\right)\right) \text {. }
\end{aligned}
$$


Corollary 5.15. A premodular category $\mathcal{B}$ has property $F$ if and only if any of its ribbon zestings $\mathcal{B}^{(\lambda, j, t)}$ has property $F$.

Proof. Any premodular category can be included in a modular category, e.g., in its Drinfeld center. Then, it follows from Vafa's theorem [1, Theorem 3.1.19], [25] that the double braiding in any premodular has finite order. Then $j_{a}\left(X_{a}\right) t(a, a)$ is a root of unity, and $\rho_{n}^{\lambda}$ has finite image if and only if $\rho_{n}$ has finite image.

\section{Applications}

We now apply the theory of zesting to a number of familiar examples.

6.1. Braided zesting of modular tensor categories. We will apply the obstruction developed in the last section to our main case of interest. Let $\mathcal{B}$ be a modular category and $A=U(\mathcal{B})$ the universal grading. The maximal pointed fusion category in $\mathcal{B}_{a d}=\mathcal{B}_{e}$ is centralized by the subcategory $\mathcal{B}_{p t}$ generated by invertible objects [14, Corollary 8.22.7]. In particular $\mathcal{B}_{a d} \cap \mathcal{B}_{p t}$ is a pointed symmetric fusion category.

Recall that every symmetric pointed fusion category has the form $\operatorname{Vec}_{S}^{\nu}$, where $S$ is an abelian group, $\nu: S \rightarrow \mathbb{Z} / 2$ is an additive group homomorphism and the braiding is given by

$$
c(a, b)=(-1)^{\nu(a) \nu(b)} \mathrm{id}_{a \otimes b} .
$$

In particular, $\operatorname{Vec}_{S}^{\nu}$ is super-Tannakian in general, and Tannakian if $\nu$ is trivial.

Proposition 6.1. Let $\mathcal{B}$ be a braided fusion category graded by $A$. If $\operatorname{Vec}_{S}^{\nu} \subset\left(\mathcal{B}_{e}\right)_{p t}$, then for every $\lambda \in Z^{2}(A, S)$ the obstruction to the existence of an associative zesting is given by

$$
O_{4}\left(a_{1}, a_{2}, a_{3}, a_{4}\right)=\left(\lambda \cup_{\nu} \lambda\right)\left(a_{1}, a_{2}, a_{3}, a_{4}\right)=(-1)^{\nu\left(\lambda\left(a_{1}, a_{2}\right) \nu\left(\lambda\left(a_{3}, a_{4}\right)\right)\right.} .
$$

In particular, if $S$ has odd order the obstruction automatically vanishes.

Remark 6.2. In practice, we overcome this obstruction by finding a $\lambda \in C^{3}\left(A, \mathbb{k}^{\times}\right)$ so that $\delta(\lambda)\left(a_{1}, a_{2}, a_{3}, a_{4}\right)=O_{4}\left(a_{1}, a_{2}, a_{3}, a_{4}\right)$. If $|S|$ is odd $O_{4}\left(a_{1}, a_{2}, a_{3}, a_{4}\right) \equiv 1$ and we may choose any 3 -cocycle $\lambda$ (e.g. $\lambda \equiv 1$ ), whereas if this obstruction does not vanish identically we must solve the linear system coming from $\delta(\lambda)\left(a_{1}, a_{2}, a_{3}, a_{4}\right)=$ $O_{4}\left(a_{1}, a_{2}, a_{3}, a_{4}\right)$. Here the 3-cochain $\lambda$ corresponds to the scalar associated with the map $\lambda_{a_{1}, a_{2}, a_{3}}: \lambda\left(a_{1}, a_{2}\right) \lambda\left(a_{1} a_{2}, a_{3}\right) \rightarrow \lambda\left(a_{1}, a_{2} a_{3}\right) \lambda\left(a_{2}, a_{3}\right)$.

\subsection{Cyclic zesting.}

6.2.1. Cohomology of cyclic groups. Let $C=\langle g\rangle$ be a cyclic group of order $N$ and $M$ an abelian group. We will identify $C$ with $\mathbb{Z} / N$, via the isomorphism $\mathbb{Z} / N \rightarrow C, a \mapsto$ 
$g^{a}$. We define some cochains associated with any $\nu \in M$ that will be useful later.

$$
\begin{aligned}
\beta_{\nu}(i) & =i \nu, \\
\gamma_{\nu}(i, j) & =\left\{\begin{array}{ll}
0 & \text { if } i+j<N \\
\nu & \text { if } i+j \geq N
\end{array},\right. \\
\lambda_{\nu}(i, j, k) & =\left\{\begin{array}{ll}
0 & \text { if } i+j<N \\
k \nu & \text { if } i+j \geq N
\end{array},\right.
\end{aligned}
$$

where $0 \leq i, j, k \leq N$. By a straightforward computation by cases, we have that

$$
\begin{aligned}
\delta\left(\beta_{\nu}\right) & =\gamma_{N \nu} \\
\delta\left(\gamma_{\nu}\right) & =0 \\
\delta\left(\lambda_{\nu}\right)(i, j, k, l) & = \begin{cases}N \nu & \text { if } i+j, k+l \geq N, \\
0 & \text { otherwise. }\end{cases}
\end{aligned}
$$

Hence, $\gamma_{\nu} \in Z^{2}(\mathbb{Z} / N, M)$, and $\gamma_{N \nu} \in B^{2}(\mathbb{Z} / N, M)$. Moreover for all $\nu \in M_{N}:=$ $\{m \in M: N m=0\}$ we have $\lambda_{\nu} \in Z^{3}(\mathbb{Z} / N, M)$. It is well known that the induced group homomorphisms

$$
\gamma: M / N M \rightarrow H^{2}(\mathbb{Z} / N, M), \quad \lambda: M_{N} \rightarrow H^{3}(\mathbb{Z} / N, M)
$$

are in fact group isomorphisms, see for more details [26].

6.2.2. Braided pointed fusion categories from cyclic groups. Let $\mathcal{C}(C, \Theta)$ be a braided pointed fusion category with $\operatorname{Inv}(\mathcal{B})=\langle g\rangle=C$ a cyclic group of order $N$ and ribbon structure $\Theta(g)$ such that $\operatorname{dim}(a)=1$ for all $a \in C$. We have that $\operatorname{Inv}\left(\mathcal{Z}_{2}(\mathcal{C}(C, \Theta))\right)=$ $\left\langle g^{m}\right\rangle$, where $m=\operatorname{Ord}\left(\Theta_{g}^{2}\right)$, and

(i) $\mathcal{C}(C, \Theta)$ is modular if and only if $\Theta_{g}^{2} \in \mathbb{k}^{\times}$has order $N$.

(ii) $\mathcal{C}(C, \Theta)$ is symmetric if and only if $\Theta_{g}^{2}=1$, and this case

(a) $\mathcal{C}(C, \Theta)$ is Tannakian if and only if $\Theta_{g}=1$

(b) $\mathcal{C}(C, \Theta)$ is super-Tannakian if and only if $\Theta_{g}=-1$.

In the symmetric case the ribbon is a character (trivial in the Tannakian case), and the braiding can be described as

$$
c_{g_{1}, g_{2}}= \begin{cases}-\mathrm{id}_{g_{1} \otimes g_{2}}, & \text { if } \Theta_{g_{1}}=\Theta_{g_{2}}=-1, \\ \operatorname{id}_{g_{1} \otimes g_{2}}, & \text { otherwise. }\end{cases}
$$

6.2.3. Cyclic braided zestings. In this section we fix $\mathcal{B}$ a braided fusion category and $C \subset \operatorname{Inv}(\mathcal{B})$ a cyclic group of order $N$ such that $\chi: C \rightarrow \operatorname{Aut}_{\otimes}\left(\operatorname{Id}_{\mathcal{B}}\right)$ is injective (equivalently, $C$ contains no non-trivial transparent objects). By Corollary 2.9. $\mathcal{B}$ has a faithful $\widehat{C}$-grading

$$
\mathcal{B}_{\gamma}=\left\{X: \chi_{a}(X)=\gamma(a) \operatorname{id}_{X}, \quad \forall a \in C\right\}, \quad \gamma \in \widehat{C} .
$$


Then $\mathcal{B}_{1} \cap C=\operatorname{ker}\left(\chi_{C, C}\right)=\mathcal{Z}_{2}(C)$, where $1 \in \widehat{C}$ is the trivial character and $\operatorname{ker}\left(\chi_{C, C}\right)=\left\{a \in C: \chi_{a}(b)=1, \forall b \in C\right\}$. We are interested in describing the braided $\widehat{C}$-zesting induced by elements in $H^{2}\left(\widehat{C}, \mathcal{Z}_{2}(C)\right)$.

Let $g \in C$ be a generator. Thus $\widehat{C}$ can canonically be identified with $\left\{\mu \in \mathbb{k}^{\times}\right.$: $\left.\mu^{N}=1\right\}$, via $\gamma \mapsto \gamma(g)$. Hence, in order fix a generator of $\widehat{C}$, from now we fix a primitive root of unity $q \in \mathbb{k}^{\times}$. Under the isomorphism $\mathbb{Z} / N \rightarrow \widehat{C}, a \mapsto\left[g^{b} \mapsto q^{a b}\right]$, we have that $\mathcal{B}$ is $\mathbb{Z} / N$-graded with

$$
\mathcal{B}_{a}=\left\{X: \chi_{g}(X)=q^{a} \operatorname{id}_{X}, \quad \forall a \in C\right\}, \quad a \in \mathbb{Z} / N
$$

Let

$$
h:=g^{\left[C: \mathcal{Z}_{2}(C)\right]}
$$

be a generator of $\mathcal{Z}_{2}(C)$ and $\Theta: \mathcal{Z}_{2}(C) \rightarrow \mathbb{k}^{\times}$be the canonical ribbon twist.

6.2.4. Associative zestings. With the aim of unifying our results and formulas we define $\epsilon \in\{1,0\}$ depending on an integer $a \in \mathbb{Z}$ and $\Theta_{h} \in\{ \pm 1\}$ as

$$
\epsilon= \begin{cases}1 & \text { if } \Theta_{h^{a}}=-1, \\ 0 & \text { if } \Theta_{h^{a}}=1 .\end{cases}
$$

Additionally, we fix $\zeta \in \mathbb{k}^{\times}$a primitive root of unity of order $2 N$ such that $\zeta^{2}=q$.

Proposition 6.3. The equivalence classes of associative zestings related with $H^{2}\left(\widehat{C}, \mathcal{Z}_{2}(C)\right)$ are parametrized by $\mathbb{Z} / m \times \mathbb{Z} / N$. The associative zesting corresponding to a pair $(a, b) \in \mathbb{Z} / m \times \mathbb{Z} / N$ is given by

$$
\begin{array}{r}
\lambda_{a}(i, j)= \begin{cases}1 & \text { if } i+j<N \\
h^{a} & \text { if } i+j \geq N\end{cases} \\
\lambda_{b}(i, j, k)= \begin{cases}1 & \text { if } i+j<N \\
\zeta^{k(\epsilon+2 b)} & \text { if } i+j \geq N\end{cases}
\end{array}
$$

where $0 \leq i, j, k<N, m=\left|\mathcal{Z}_{2}(C)\right|, \zeta^{2}=q$, and $\epsilon$ is defined in (6.11).

Proof. Since $\mathcal{Z}_{2}(C)$ has order $m$, the 2-cocycles $\lambda_{a}$ with $a \in \mathbb{Z} / m$ form a set of representatives of $H^{2}\left(\mathbb{Z} / N, \mathcal{Z}_{2}(C)\right)$.

If $\Theta_{h}=1$, we have that $\mathcal{Z}_{2}(C)$ is Tannakian and then the 4-cocycle obstruction $\mathrm{O}_{4}\left(\lambda_{a}\right)$ automatically vanishes. Hence associative zestings are parametrized by pair $(a, b) \in \mathbb{Z} / m \times \mathbb{Z} / N$, with corresponding zesting

$$
\begin{gathered}
\lambda_{a}(i, j)= \begin{cases}\mathbf{1} & \text { if } i+j<N \\
h^{a} & \text { if } i+j \geq N\end{cases} \\
\lambda_{b}(i, j, k)= \begin{cases}1 & \text { if } i+j<N \\
q^{k b} & \text { if } i+j \geq N\end{cases}
\end{gathered}
$$


where $0 \leq i, j, k<N$.

If $\Theta_{h}=-1$, we have that $\mathcal{Z}_{2}(C)$ is super-Tannakian. In particular $\mathcal{Z}_{2}(C)$ has even order, and let $v: \mathcal{Z}_{2}(C) \rightarrow \mathbb{Z} / 2$ the non-trivial group homomorphism. The 4-cocycle obstruction $\mathrm{O}_{4}\left(\lambda_{a}\right)$ is given by

$$
\begin{aligned}
O_{4}(i, j, k, l) & =(-1)^{v\left(\lambda_{a}(i, j)\right) v\left(\lambda_{a}(k, l)\right)} \\
& = \begin{cases}(-1)^{a} & \text { if } i+j, k+l \geq N \\
1 & \text { otherwise. }\end{cases}
\end{aligned}
$$

Note that the set of all solutions to the equation $Q^{N}=-1$ can be parametrized as $\zeta^{1+2 b}$, with $b \in \mathbb{Z} / N$. Hence, for $a$ odd using $(\underline{6.6})$ we have that the associative zestings is parametrized by pairs $(a, b) \in \mathbb{Z} / m \times \mathbb{Z} / N$, with

$$
\begin{array}{r}
\lambda_{a}(i, j)= \begin{cases}\mathbf{1} & \text { if } i+j<N \\
h^{a} & \text { if } i+j \geq N\end{cases} \\
\lambda_{b}(i, j, k)= \begin{cases}1 & \text { if } i+j<N \\
\zeta^{k(1+2 b)} & \text { if } i+j \geq N\end{cases}
\end{array}
$$

where $0 \leq i, j, k<N$.

\subsubsection{Braided zestings.}

Proposition 6.4. Let $(a, b) \in \mathbb{Z} / m \times \mathbb{Z} / N$ and $\left(\lambda_{a}, \lambda_{b}\right)$ the associative zesting constructed in Proposition 6.3. Then

(i) $\left(\lambda_{a}, \lambda_{b}\right)$ admits a braided zesting with $j=$ id if and only if $a \frac{N}{m}=\epsilon+2 b \bmod N$.

(ii) There are $N$ different braided zesting parametrized by $\left\{s \in \mathbb{k}^{\times}: s^{N}=\zeta^{-(\epsilon+2 b)}\right\}$.

(iii) The braided zesting associated with $s$, is given by $j_{y}=\operatorname{Id}_{\mathcal{B}}$ for all $y \in \mathbb{Z} / N$ and

$$
t_{s}(i, j)=s^{-i j} \operatorname{id}_{\lambda_{a}(i, j)}, \quad 0 \leq i, j<N .
$$

Proof. In order to compute the 2-cocycles $O_{1}\left(\lambda_{b}\right)$, we can take initially $t(i, j)=$ $\operatorname{id}_{\lambda_{a}(i, j)}$. Then

$$
O_{1}\left(\lambda_{b}\right)(i \mid j, k)=\frac{\lambda_{b}(i, j, k) \lambda_{b}(j, k, i)}{\lambda_{b}(j, i, k)}=\lambda_{b}(j, k, i)
$$

Since $H^{2}\left(\mathbb{Z} / N, \mathbb{k}^{\times}\right)=0$, we can redefine the isomorphisms

$$
t(i, j)=l(i, j) \operatorname{id}_{\lambda(i, j)}, \quad l(i, j) \in \mathbb{k}^{\times} . \quad i, j \in \mathbb{Z} / N,
$$

to that satisfy the equation in Figure 7. We need to choose $s$ so that $s^{N}=\zeta^{-(\epsilon+2 b)}$. Then define

$$
l(i, j)=s^{-i j}
$$

where $0 \leq i, j, k<N$. In fact, by (6.4)

$$
\frac{l(i, k) l(j, k)}{l(i+j, k)}=\delta\left(\beta_{s}\right)^{k}(i, j)=\lambda_{b}(i, j, k)^{-1}
$$


and since $l(i, j)=l(j, i)$, we have that

$$
\frac{l(i, k) l(i, j)}{l(i, j+k)}=\lambda_{b}(j, k, i)=O_{1}\left(\lambda_{b}\right)(i \mid j, k)
$$

Now,

$$
\begin{aligned}
O_{2}(b, s)(i, j \mid k) & =\frac{l(i, k) l(j, k)}{l(i+j, k)} \lambda_{b}(i, j, k) \\
& =\lambda_{b}(i, j, k)^{2}
\end{aligned}
$$

Finally, since $\chi_{h^{a}}\left(X_{k}\right)=q^{a \frac{N}{m} k}$, we get that

$$
\chi_{\lambda_{a}(i, j)\left(X_{1}\right)} O_{2}\left(\lambda_{a}, \lambda_{b}\right)(i, j \mid 1)= \begin{cases}1 & \text { if } i+j<N \\ q^{a \frac{N}{m}-(\epsilon+2 b)} & \text { if } i+j \geq N\end{cases}
$$

that is $\left[\chi_{\lambda_{a}} / O_{2}\right]=a \frac{N}{m}-(\epsilon+2 b) \in \mathbb{Z} / N \cong H^{2}(\mathbb{Z} / N, \widehat{\mathbb{Z} / N})$. Then if $a \frac{N}{m} \neq \epsilon+2 b$ mod $N$ the associative zesting $\left(\lambda_{a}, \lambda_{b}\right)$ does not admits a braided zesting with $j=\mathrm{id}$ and if $a \frac{N}{m}=\epsilon+2 b \bmod N,\left[O_{2}(\lambda)\right]=\left[\chi_{\lambda}\right]$, so $j_{t}=\mathrm{id}$ and $t_{s}(i, j)=s^{-i j} \operatorname{id}_{\lambda_{a}(i, j)}$ define a braided zesting.

6.2.6. Ribbon zesting and its modular data.

Proposition 6.5. Let $\left(\lambda_{a}, \lambda_{b}, \mathrm{id}, t_{s}\right)$ be a braided zesting constructed in Proposition 6.4. If $\mathcal{B}$ has a ribbon twist $\theta$ such that $\theta_{h^{a}}=\Theta_{h^{a}}$ then a ribbon zesting $f: \mathbb{Z} / N \rightarrow \mathbb{K}^{\times}$ is defined by

$$
f(i)=s^{-i^{2}}, \quad 0 \leq i<N
$$

and its modular data is given by

$$
\begin{array}{cc}
T_{X_{i}, X_{i}}^{\lambda}=s^{-i^{2}} T_{X_{i}, X_{i}}, & 0 \leq i<N, X_{i} \in \mathcal{B}_{i}, \\
S_{X_{i}, Y_{j}}^{\lambda}=s^{2 i j} S_{X_{i}, Y_{j}}, & 0 \leq i, j<N, X_{i} \in \mathcal{B}_{i}, Y_{j} \in \mathcal{B}_{j} .
\end{array}
$$

Proof. Equation (5.1) is

$$
f(i) f(i+j)^{-1} f(j)=s^{2 i j} \chi_{\lambda(i, j)}(i+j) \theta_{\lambda(i, j)}= \begin{cases}s^{2 i j} & \text { if } i+j<N \\ s^{2 i j} q^{a \frac{N}{m}(i+j)} \theta_{h^{a}} & \text { if } i+j \geq N .\end{cases}
$$

Let $0 \leq i, j<N-1$. If $i+j<N$, then

$$
f(i) f(i+j)^{-1} f(j)=s^{-i^{2}} s^{(i+j)^{2}} s^{-j^{2}}=s^{2 i j} .
$$

If $i+j \geq N$, then $i+j=[i+j]+N$ where $0 \leq[i+j]<N$, and $(i+j)^{2}=$ $i^{2}+j^{2}+2 i j-2 N(i+j)+N^{2}$, thus 


$$
\begin{aligned}
f(i) f(i+j)^{-1} f(j) & =s^{2 i j-2 N(i+j)} s^{N^{2}} \\
& =s^{2 i j} q^{(\epsilon+2 b)(i+j)}(-1)^{\epsilon+2 b} \\
& =(-1)^{\epsilon} q^{a \frac{N}{m}(i+j)} s^{2 i j} \\
& =\theta_{h^{a}} q^{a \frac{N}{m}(i+j)} s^{2 i j} .
\end{aligned}
$$

Equation (5.2) for $0<i<N-1<$ is

$$
f(i) f(N-i)^{-1}=\chi_{h^{a}}(i) \theta_{h^{i}}=q^{a \frac{N}{m} i} \theta_{h^{a}}
$$

and

$$
\begin{aligned}
f(i) f(N-i) & =s^{-i^{2}} s^{(N-i)^{2}} \\
& =s^{-2 N i} s^{N^{2}}=(-1)^{\epsilon} q^{(\epsilon+2 b) i} \\
& =\theta_{h_{a}} q^{a \frac{N}{m} i} .
\end{aligned}
$$

The formulas for modular data follow from Theorem 5.7. In fact, the T-matrix follows directly from the definition of $\theta^{f}$. First note that since $\theta_{h}=\Theta_{h}$, then $\operatorname{dim}\left(h^{i}\right)=1$, in particular $\operatorname{dim}\left(\lambda_{a}(i, j)\right)=1$ for all $i, j \in \mathbb{Z} / N$, second

$$
\frac{S_{X_{i}, \lambda(j,-j)}}{\operatorname{dim}\left(X_{i}\right)}=\chi_{h^{a}}\left(X_{i}^{*}\right)=q^{-a \frac{N}{m} i}, \quad 0<i<N
$$

and third

$$
s^{-i(N-j)}=s^{2 i j} q^{(\epsilon+2 b) i}
$$

hence

$$
\begin{aligned}
S_{X_{i}, Y_{j}}^{\lambda} & =\frac{f(i-j) t^{(2)}(i,-j)}{t(i-j, i-j)} \frac{S_{X_{i}, \lambda(j,-j)}}{\operatorname{dim}\left(X_{a}\right)} S_{X_{i}, Y_{j}} \\
& =s^{-i(N-j)} \frac{S_{X_{i}, \lambda(j,-j)}}{\operatorname{dim}\left(X_{a}\right)} S_{X_{i}, Y_{j}} \\
& =s^{2 i j} S_{X_{i}, Y_{j}} .
\end{aligned}
$$

Remark 6.6. If $N$ is odd $f(i)=s^{-i^{2}}$ is the unique ribbon zesting and if $N$ is even $f(i)$ and $(-1)^{i} f(i)$ are all the ribbon zestings. From the proof of the above we see that this changes the $S$-matrix by a factor of $(-1)^{i-j}$ on the $(i, j)$-graded block.

As a particular case of Proposition 6.4 we obtain the following result on fermion zesting (cf. [4]):

Corollary 6.7. Let $\mathcal{B}$ be a braided fusion category and $f \in \mathcal{B}$ a simple object such that

(i) $f^{\otimes} f \cong 1$,

(ii) $\Theta_{f}=-1$, 
(ii) $\chi_{f}$ is not the identity.

Then $\mathcal{B}$ has eight different braided $\mathbb{Z} / 2$-settings parameterized with modular data

$$
T_{X_{i}, X_{i}}^{\lambda}=s^{-i^{2}} T_{X_{i}, X_{i}}, \quad S_{X_{i}, Y_{j}}^{\lambda}=s^{2 i j} S_{X_{i}, Y_{j}}
$$

where $s$ is a root of unit of order eight.

6.3. Quantum Group categories of type $A$. A large class of examples of modular categories satisfying the hypotheses of section 6.2.2 can be obtained from quantum groups (see [1, Section 3.3]). Of particular interest are the modular categories $S U(N)_{k}$ obtained from quantum groups $U_{Q} \mathfrak{s l}_{N}$ for $Q=e^{\pi i /(N+k)}$ (eschewing $q$ to avoid notation clashes). Two references for this construction are [3, 21, where much of what follows can be found. For any $N, k$, the category $S U(N)_{k}$ has a (maximally) pointed subcategory $\mathcal{P}(N, k)$ with fusion rules like $\mathbb{Z} / N$. In particular $S U(N)_{k}$ is (universally) $\mathbb{Z} / N$-graded with trivial component $P S U(N)_{k}:=\left[S U(N)_{k}\right]_{e}$. Labelling the fundamental weights of the root system of type $A_{N-1}$ by $\varpi_{i}$ for $i=1, \ldots, N-1$ (we follow [2, Planches, Chapters IV,V,VI] for notation), we find that the simple objects in $\mathcal{P}(N, k)$ correspond to weights 0 and $k \varpi_{i}$ for $i=1, \ldots, N-1$. For notational convenience we define $g=X_{k \varpi_{1}}$ so that $g^{0}=1$ and $g^{t}:=X_{k \varpi_{t}}$. In this notation we have $g^{i} \otimes g^{j}=g^{i+j}$. To determine the nature of this subcategory we must compute the twists $\theta_{i}:=\theta_{g^{i}}$, for which we employ standard techniques (see [1, 24], for example). The key computation is that the twist of the simple object labeled by highest weight $\mu$ is $\theta_{\mu}=Q^{\mathfrak{c}_{\mu}}$ where $\mathfrak{c}:=\langle\mu+2 \rho, \mu\rangle$. We find:

$$
\theta_{j}=\zeta_{2 N}^{k j(N-j)}, \quad \text { where } \zeta_{2 N}=e^{\pi i / N} .
$$

Thus we may identify $\mathcal{P}(N, k)$ with the pointed ribbon fusion category $\mathcal{C}(\mathbb{Z} / N, \eta)$ where $\eta$ is the quadratic form given by $\eta(j)=\theta_{j}$.

By the twist equation we obtain the formula for the double braiding in $\mathcal{P}(N, k)$ as:

$$
c_{g^{t}, g^{s}} \circ c_{g^{s}, g^{t}}=\frac{\theta_{s+t}}{\theta_{s} \theta_{t}}=\zeta_{2 N}^{-2 s t k} \mathrm{id}_{g^{s+t}}
$$

so that the Müger center of $\mathcal{P}(N, k)$ is generated by $g^{\frac{N}{(N, k)}}$. In particular, $\mathcal{P}(N, k)$ is modular if and only if $(k, N)=1$ in which case we have the factorization $S U(N)_{k} \cong$ $P S U(N)_{k} \otimes \mathcal{P}(N, k)$ as modular categories, and $\operatorname{Inv}\left(P S U(N)_{k}\right)$ is trivial.

On the other hand if $N \mid s k$ then $g^{s}$ centralizes $\mathcal{P}(N, k)$ and hence lies in the trivial component $\mathcal{B}_{0}=P S U(N)_{k}$ (under the universal $\mathbb{Z} / N$-grading). Thus $\mathcal{P}(N, k) \cap$ $P S U(N)_{k}$ is a non-trivial symmetric pointed subcategory whenever $(N, k) \neq 1$. Indeed, $\mathcal{P}(N, k)$ is symmetric if and only if $N \mid k$. Furthermore, by the form of the twists calculated above we can determine when $\mathcal{P}(N, k)$ for $k=\alpha N$ is Tannakian or super-Tannakian. If $N$ is odd, we only have Tannakian categories, but if $N$ is even, we have that $\mathcal{P}(N, \alpha N)$ is Tannakian if and only if $\alpha$ is even, and super-Tannakian otherwise. 
The object $X_{1}$ labelled by the highest weight $\varpi_{1}$ is a tensor generator for $S U(N)_{k}$. We will assume that $X_{1}$ is in the 1 -graded component of the universal $\mathbb{Z} / N$-grading $\mathcal{B}_{1}$. Applying Proposition 2.8 (iv) we first compute $\chi: U(\mathcal{B})=\mathbb{Z} / N \rightarrow \widehat{\mathbb{Z} / N}$. Now $\chi_{g^{a}}$ is determined by $\chi_{g^{a}}(1)$ since $\chi_{g^{a}}(m)=\chi_{g^{a}}(1)^{m}$, and $\chi_{g^{a}}(1)=\left(\chi_{g}(1)\right)^{a}$ since the operation on $\widehat{\mathbb{Z} / N}$ is pointwise. Thus we reduce to computing the scalar associated with the double braiding $c_{g, X_{1}} \circ c_{X_{1}, g}$ where $X_{1}=X_{\varpi_{1}}$. As $g \otimes X_{1}=X_{(k-1) \varpi_{1}+\varpi_{2}}$ is simple we need only compute

$$
c_{g, X_{1}} \circ c_{X_{1}, g}=\frac{\theta_{(k-1) \varpi_{1}+\varpi_{2}}}{\theta_{1} \theta_{\varpi_{1}}}=\zeta_{N}^{-1}
$$

where $\zeta_{N}:=e^{2 \pi i / N}$. Thus we see that $\chi_{g^{a}}(m)=q^{a m}$ where $q:=\zeta_{N}^{-1}$. In this way the $\mathbb{Z} / N$-grading is given by $\mathcal{B}_{i}=\left\{X: \chi_{g}(X)=q^{i} \operatorname{id}_{X}\right\}$ as in (6.9). Notice that $q$ is determined once we declare that $X_{1} \in \mathcal{B}_{1}$ and pick our generator $g$ of $U(\mathcal{B})$-fixing any two choices among the grading, $q$ and generator of $U(\mathcal{B})$ determine the third.

Now we may apply the results of subsection 6.1 to $S U(N)_{k}$. We will consider several cases to illustrate the subtleties:

(1) For $S U(N)_{\alpha N}$ for $N$ odd the pointed subcategory $\mathcal{P}(N, \alpha N)$ is Tannakian. We will zest with respect to the universal $\mathbb{Z} / N$-grading so that $m=N$ and $\epsilon=0$ in the notation of Proposition 6.3 . Thus there are $N^{2}$ associative zestings $\left(\lambda_{a}, \lambda_{b}\right)$ where $(a, b) \in \mathbb{Z} / N \times \mathbb{Z} / N$. The $N$ associative zestings for pairs $(2 b, b)$ each admit $N$ braided zestings, which in turn admit $N$ twist braided zestings. Thus there are at most a total of $N^{2}$ distinct ribbon twist braided zestings, all of which are modular by Proposition 5.11.

(2) For $S U(N)_{\alpha N}$ with $N$ even and $\alpha$ odd the pointed subcategory $\mathcal{P}(N, \alpha N)$ is super-Tannakian. We will zest with respect to the universal $\mathbb{Z} / N$-grading so that $m=N$ in the notation of Proposition 6.3. Thus there are $N^{2}$ distinct associative zestings $\left(\lambda_{a}, \lambda_{b}\right)$ where $(a, b) \in \mathbb{Z} / N \times \mathbb{Z} / N$. For $a$ even we have $\epsilon=$ 0 and the situation is similar as above: the $N$ associative zestings $\left(\lambda_{a}, \lambda_{a / 2}\right)$ and $\left(\lambda_{a}, \lambda_{(a+N) / 2}\right)$ each admit $N$ braided zestings and $N^{2}$ twist braided zestings. Among these $2 N$ of them are ribbon twist zestings, all of which are modular by Proposition 5.11. Now for $a$ odd we have $\epsilon=1$ so that the $N$ pairs $\left(a, \frac{a-1}{2}\right)$ and $\left(a, \frac{a-1+N}{2}\right)$ admit $N$ braided zestings each. All told there are at most $4 N^{2}$ ribbon braided zestings, each of which is modular.

We hasten to point out that, in practice, there can be equivalences among braided zestings. We will see some examples of this below.

6.3.1. $S U(3)_{3}$. Consider the non-group-theoretical [23] integral modular category $S U(3)_{3}$ of rank 10 and dimension 36 (this example inspired the notion of zesting in [5]). We define $q=e^{-2 \pi i / 3}$ and $\zeta=e^{2 \pi i / 18}$, and order the simple objects as follows $\left[\mathbf{1}, g, g^{2}, Y, X_{1}, X_{2}, X_{3}, Z_{1}, Z_{2}, Z_{3}\right]$. In the correspondence with the $S U(3)$ highest weights we have $X_{1}$ labeled by $\varpi_{1}$ and $g$ by $3 \varpi_{1}$ as above. Then $X_{2}=g \otimes X_{1}$ and $X_{3}=g^{2} \otimes X_{1}$, with $Z_{i}=X_{i}^{*}$ in $S U(3)_{3}$. We have $\operatorname{dim}(Y)=3, \operatorname{dim}\left(X_{i}\right)=\operatorname{dim}\left(Z_{i}\right)=2$ for all $i$, and the $\mathbb{Z} / 3$-grading is given by $X_{i} \in \mathcal{B}_{1}$ and $Z_{i} \in \mathcal{B}_{2}$. The twists of the 
simple objects ordered as above are $\left[1,1,1,-1, \zeta^{4}, \zeta^{16}, \zeta^{10}, \zeta^{4}, \zeta^{16}, \zeta^{10}\right]$. We note that there are two inequivalent sets of modular data $(S, T)$ (and hence, presumably, modular categories) with these fusion rules: the above and its complex conjugate. Notice that the twists of the $X_{i}$ are primitive 9th roots of unity, so that there are $6 \mathrm{Ga}$ lois conjugates. However, the relabeling symmetry among the pairs $\left(X_{i}, Z_{i}\right)$ allows us to recognize these 6 conjugates as belonging to just 2 inequivalent classes. The (unnormalized) modular $S$-matrix has the block $3 \times 3$ form: $S=\left(\begin{array}{ccc}A & B & \bar{B} \\ B^{T} & C & D \\ \bar{B}^{T} & D^{T} & C\end{array}\right)$

where

$$
\begin{gathered}
A=\left(\begin{array}{cccc}
1 & 1 & 1 & 3 \\
1 & 1 & 1 & 3 \\
1 & 1 & 1 & 3 \\
3 & 3 & 3 & -3
\end{array}\right), \quad B=2\left(\begin{array}{ccc}
1 & 1 & 1 \\
\zeta^{6} & \zeta^{6} & \zeta^{6} \\
-\zeta^{6} & -\zeta^{6} & -\zeta^{6} \\
0 & 0 & 0
\end{array}\right), \\
C=2\left(\begin{array}{ccc}
\zeta & \zeta^{7} & -\zeta^{4} \\
\zeta^{7} & -\zeta^{4} & \zeta \\
-\zeta^{4} & \zeta & \zeta^{7}
\end{array}\right), \quad \text { and } D=2\left(\begin{array}{ccc}
\zeta^{2}-\zeta^{5} & \zeta^{8}-\zeta^{5} & \zeta^{8}+\zeta^{2} \\
\zeta^{8}-\zeta^{5} & \zeta^{8}+\zeta^{2} & \zeta^{2}-\zeta^{5} \\
\zeta^{8}+\zeta^{2} & \zeta^{2}-\zeta^{5} & \zeta^{8}-\zeta^{5}
\end{array}\right) .
\end{gathered}
$$

The 9 associative zestings of $S U(3)_{3}$ are parameterized by $(a, b) \in \mathbb{Z} / 3 \times \mathbb{Z} / 3$ where

$$
\lambda_{a}(i, j)=\left\{\begin{array}{ll}
1, & i+j<3 \\
g^{a} & i+j \geq 3
\end{array} \text { and } \quad \lambda_{b}(i, j, k)=\left\{\begin{array}{ll}
1, & i+j<3 \\
q^{b k} & i+j \geq 3
\end{array} .\right.\right.
$$

The fusion rules for $a=2$ and $a=0$ are the isomorphic: reordering the simple objects as $\left[\mathbf{1}, g^{2}, g, X_{1}, X_{3}, X_{2}, Z_{3}, Z_{2}, Z_{1}\right]$ gives us the fusion rule isomorphism. By results of 21] the 6 fusion categories corresponding to $a=0$ and $a=2$ are obtained from $S U(3)_{3}$ by changing the quantum parameter $Q$ and/or changing the associativity by a 3-cocycle. On the other hand, for $a=1$ we find that $\mathbf{1} \not \subset X_{1}^{\otimes_{1} 3}$ yet $\mathbf{1} \subset X^{\otimes 3}$ for all simple objects $X \in S U(3)_{3}$ so that these fusion rules are not isomorphic to those of $S U(3)_{3} \frac{1}{1}$

Now for each pair $(a, b) \in\{(0,0),(1,2),(2,1)\}$ we obtain 3 braided zestings by choosing an $s$ so that $s^{3}=q^{-b}$, by Proposition 6.4. Moreover, by Proposition 6.5 these each have a unique ribbon zesting, given by multiplying the $S U(3)_{3}$ twists in the component $\mathcal{B}_{i}$ by $s^{-i^{2}}$, and all are modular with these choices. Thus there are at most 9 modular categories obtained from $\mathbb{Z} / 3$-zesting of $S U(3)_{3}$. In fact, we will see that there are only 3 inequivalent sets of modular data, and presumably only 3 inequivalent modular categories (this is not immediate as modular data is not a complete invariant, see [22].

For $b=0$ we have $s^{3}=1$ and for $b=1$ we have $s^{3}=q^{-1}=e^{2 \pi i / 3}$. The twist on $\mathcal{B}_{1}$ and $\mathcal{B}_{2}$ are primitive 9 th roots of unity $\left\{\zeta^{4}, \zeta^{10}, \zeta^{16}\right\}$, so that rescaling by $s^{-1}=s^{-4}$ with $s=e^{2 \pi i n / 3}$ simply permutes them. Similarly for the case $b=2$ we have $s=\zeta^{6 x+2}$ for $0 \leq x \leq 2$ so that rescaling these twists by $s^{-1}$ and $s^{-4}$ conjugates the set of values,

\footnotetext{
${ }^{1}$ It seems to be the case that the trivial representation appears in $X_{\mu}^{\otimes N}$ for any object $X_{\mu} \in$ $\operatorname{Rep}\left(\mathfrak{s l}_{N}\right)$, but we could not find a proof in the literature.
} 
and permutes them in a way consistent with the fusion rule isomorphism above. Now since the fusion rules and dimensions are the same, the $S$-matrices are determined by the twist (via the balancing equation). Thus we obtain two sets of modular data from the pairs $(0,0)$ and $(1,2)$ : those of $S U(3)_{3}$ and the complex conjugate. Indeed, it is easily checked that adjusting the $S U(3)_{3} S$-matrix above by a factor of $s^{2 i j}$ on the $(i+1, j+1)$ block has the effect of permuting the rows/columns and possibly complex conjugating the entries.

Now for $b=2$ we have $s^{3}=q^{-2}=e^{-2 \pi i / 3}$, with solutions $s=e^{-(6 x+2) \pi i / 9}=\zeta_{9}^{-3 x-1}$ for $0 \leq x \leq 2$, where $\zeta_{9}:=e^{2 \pi i / 9}$. Rescaling $\left\{\zeta^{4}=\zeta_{9}^{2}, \zeta^{10}=\zeta_{9}^{5}, \zeta^{16}=\zeta_{9}^{8}\right\}$ by $s^{-1}=\zeta_{9}^{3 x+1}$ and $s^{-4}=\zeta_{9}^{12 x+4}$ both yield $\left\{1, q, q^{-1}\right\}$ for any $x$ which is invariant under complex conjugation. Again, the $S$-matrix is determined by the twists and the fusion rules by the balancing equation so that we find that there is exactly one set of modular data $(S, T)$ corresponding to the modular zesting of $S U(3)_{3}$ when $a=1$. For completeness we provide explicit modular data $(\tilde{S}, \tilde{T})$ (cf. [5, Section $4.2])$ : taking $x=0$, the twists are given by $\tilde{T}:=\left[1,1,1,-1, q^{-1}, 1, q, q, q^{-1}, 1\right]$ and $\tilde{S}=\left(\begin{array}{ccc}A & B & \bar{B} \\ B^{T} & \tilde{C} & \tilde{D} \\ \bar{B}^{T} & \tilde{D}^{T} & \tilde{C}\end{array}\right)$ where $A$ and $B$ are the same as for $S U(3)_{3}$ above and

$$
\tilde{C}=2\left(\begin{array}{ccc}
\zeta^{-3} & \zeta^{3} & -1 \\
\zeta^{3} & -1 & \zeta^{-3} \\
-1 & \zeta^{-3} & \zeta^{3}
\end{array}\right), \quad \text { and } \quad \tilde{D}=2\left(\begin{array}{ccc}
-1 & \zeta^{3} & \zeta^{-3} \\
\zeta^{3} & \zeta^{-3} & -1 \\
\zeta^{-3} & -1 & \zeta^{3}
\end{array}\right)
$$

Note that $\zeta^{ \pm 3}=-q^{ \pm 1}$ so that the entries of $\tilde{S}$ lie in the field $\mathbb{Q}(q)$.

Let us compare the zesting of $S U(3)_{3}$ to gauging constructions. Clearly $S U(3)_{3}$, its complex conjugate and its Grothendieck inequivalent zesting each contain $\operatorname{Rep}(\mathbb{Z} / 3)$ as a Tannakian subcategory. If we take the corresponding $\mathbb{Z} / 3$-condensation [10] we obtain a modular category $\mathcal{L}$ of dimension $4=36 / 3^{2}$ that has a gaugable symmetry $\phi: \mathbb{Z} / 3 \rightarrow \operatorname{Aut}_{\otimes}^{b r}(\mathcal{L})[8$. It is not difficult to see that $\mathcal{L}$ must be the so-called 3 fermion modular category $3 F$, with fusion rules like $\mathbb{Z} / 2 \times \mathbb{Z} / 2$, and the $\mathbb{Z} / 3$ action cyclically permutes the fermions. Thus we should be able to recover the three zestings of $S U(3)_{3}$ by gauging this symmetry. The obstructions to gauging vanish as they lie in $H^{3}(\mathbb{Z} / 3, \mathbb{Z} / 2 \times \mathbb{Z} / 2)=0$ and $H^{4}(\mathbb{Z} / 3, U(1))=0$. The gaugings of $3 F$ are parameterized by $H^{2}(\mathbb{Z} / 3, \mathbb{Z} / 2 \times \mathbb{Z} / 2)=0$ and $H^{3}(\mathbb{Z} / 3, U(1)) \cong \mathbb{Z}_{3}$. Thus we obtain 3 such gaugings, consistent with the zesting calculation above.

6.3.2. $S U(4)_{4}$. The rank 35 modular category $\mathcal{B}:=S U(4)_{4}$ has pointed subcategory $\mathcal{P}(4,4)$ with fusion rules like $\mathbb{Z} / 4$, but is non-Tannakian: the generator $g$ of the group $\operatorname{Inv}(\mathcal{B})$ has twist $\theta_{g}=-1$, and $c_{g, g}=-\mathrm{id}_{g^{2}}$. We write

$$
S U(4)_{4}=\mathcal{C}_{0} \oplus \mathcal{C}_{1} \oplus \mathcal{C}_{2} \oplus \mathcal{C}_{3}
$$

to decompose the category into its $\mathbb{Z} / 4$ universally-graded components, which have the following ranks 


\begin{tabular}{c|cccc} 
Component & $\mathcal{C}_{0}$ & $\mathcal{C}_{1}$ & $\mathcal{C}_{2}$ & $\mathcal{C}_{3}$ \\
\hline Rank & 10 & 8 & 9 & 8
\end{tabular}

We set $q=e^{-2 \pi i / 4}$ so that $\chi_{g}\left(X_{i}\right)=q^{i}$ for $X_{i} \in \mathcal{C}_{i}$. Table 2 summarizes the last few pages of analysis and records the parametrization of simple objects in $S U(4)_{4}$ along with their universal grading, quantum dimensions, and twists.

We first consider the $\mathbb{Z} / 4$-zestings. To conform with the notation of Propositions 6.3 and 6.4, we set $\zeta=e^{-2 \pi i / 8}$. The associative $\mathbb{Z} / 4$-zestings are parameterized by $(a, b) \in \mathbb{Z} / 4 \times \mathbb{Z} / 4$ as above. When $a$ is odd we have $\epsilon=1$ and otherwise $\epsilon=0$. Braided zestings exist for the 8 pairs

$$
(a, b) \in\{(0,0),(0,2),(1,0),(1,2),(2,1),(2,3),(3,1),(3,3)\},
$$

and are parameterized by solutions to $s^{4}=\zeta^{-\epsilon(a)-2 b}$. Each of these, in turn have a unique ribbon structure that gives positive dimensions, and each of these are modular by Lemma 5.12. Thus there are at most 32 distinct modular categories obtained as $\mathbb{Z} / 4$-zestings of $S U(4)_{4}$. For any triple $(a, b, s)$ the central charge of the corresponding modular categories are the same for any of the 4 choices of $s$, giving us (at least) 8 distinct modular categories see Table 1. As can be seen from the data in table 2, $S U(4)_{4}$ has a high degree of symmetry there are many objects of the same dimension giving rise to labeling ambiguities. Moreover, [11] shows that the group of (not necessarily braided) monoidal autoequivalences is isomorphic to $\mathbb{Z} / 2 \times \mathbb{Z} / 4$. In particular, distinguishing or identifying the the modular categories with the same underlying fusion category (i.e. the same $(a, b)$ but different $s$ ) is a subtle problem.

\begin{tabular}{|c|c|c|c|}
\hline$(a, b)$ & $X_{1}^{\otimes 4} \supset \mathbf{1 ?}$ & $s$ & central charge \\
\hline \hline$(0,0)$ & yes & $\gamma$ & $\zeta_{16}^{-1}$ \\
\hline$(0,2)$ & yes & $\gamma \cdot\left(\zeta_{32}\right)^{4}$ & $-\zeta_{16}^{-1}$ \\
\hline$(1,0)$ & no & $\gamma \cdot\left(\zeta_{32}\right)$ & $-i \cdot \zeta_{16}$ \\
\hline$(1,2)$ & no & $\gamma \cdot\left(\zeta_{32}\right)^{5}$ & $i \cdot \zeta_{16}$ \\
\hline$(2,1)$ & no & $\gamma \cdot\left(\zeta_{32}\right)^{2}$ & $-i \cdot \zeta_{16}^{-1}$ \\
\hline$(2,3)$ & no & $\gamma \cdot\left(\zeta_{32}\right)^{6}$ & $i \cdot \zeta_{16}^{-1}$ \\
\hline$(3,1)$ & yes & $\gamma \cdot\left(\zeta_{32}\right)^{3}$ & $-\zeta_{16}$ \\
\hline$(3,3)$ & yes & $\gamma \cdot\left(\zeta_{32}\right)^{7}$ & $\zeta_{16}$ \\
\hline
\end{tabular}

TABLE 1. $S U(4)_{4}$ ribbon zesting data: $\zeta_{N}:=e^{2 \pi i / N}$ and $\gamma^{4}=1$

We may also consider the $\mathbb{Z} / 2$-zestings. We can define a $\mathbb{Z} / 2$-grading on $\mathcal{B}:=$ $S U(4)_{4}$ by $\mathcal{B}_{0}=\mathcal{C}_{0} \oplus \mathcal{C}_{2}$ and $\mathcal{B}_{1}=\mathcal{C}_{1} \oplus \mathcal{C}_{3}$ where $\mathcal{C}_{i}$ are the components of the universal grading above. This corresponds to the grading by the subgroup $\mathbb{Z} / 2 \cong\left\langle g^{2}\right\rangle<\operatorname{Inv}(\mathcal{B})$. If we choose a 2 -cocycle $\lambda_{a} \in H^{2}(\mathbb{Z} / 2, \operatorname{Inv}(\mathcal{B}))$ with values in $\left\langle g^{2}\right\rangle$ then $\mathcal{Z}_{2}\left(\left\langle g^{2}\right\rangle\right)=\left\langle g^{2}\right\rangle$ so we may apply the results of Propositions 6.3 and 6.4 to obtain braided zestings and ribbon twists as above. 
On the other hand, we may define a 2-cocycle $H^{2}(\mathbb{Z} / 2, \operatorname{Inv}(\mathcal{B}))$ by $\lambda(1,1)=g$ and $\lambda(0,1)=\lambda(1,0)=1$. The normalized 3-cochains $\lambda_{ \pm}(1,1,1)= \pm i$ provide associative zestings. We claim that these fusion categories do not admit braided zestings.

We have that $\chi_{g} \mid \mathcal{B}_{0}=\operatorname{Id}_{\mathcal{C}_{0}}-\operatorname{Id}_{\mathcal{C}_{2}}$. In particular $\chi_{g}$ is not in $\operatorname{Aut}_{\otimes}^{\mathbb{Z} / 2}\left(\operatorname{Id}_{\mathcal{B}}\right)$, so that $j_{a}=\mathrm{id}$ does not satisfy condition (BZ1). What is required is a function $j: \mathbb{Z} / 2 \rightarrow$ $\mathrm{Aut}_{\otimes}\left(\operatorname{Id}_{\mathcal{B}}\right)$ such that

$$
\chi_{\lambda(a, b)} \circ j_{a b} \circ j_{a}^{-1} \circ j_{b}^{-1} \in \mathrm{Aut}_{\otimes}^{\mathbb{Z} / 2}\left(\operatorname{Id}_{\mathcal{B}}\right) .
$$

In particular taking $a=b=1 \in \mathbb{Z} / 2$ we seek a $j_{1}$ such that $\chi_{g} \circ j_{1}^{-2} \in \operatorname{Aut}_{\otimes}^{\mathbb{Z} / 2}\left(\operatorname{Id}_{\mathcal{B}}\right)$. This means that $\chi_{g} \circ j_{1}^{-2}$ must be the identity on $\mathcal{B}_{0}$, so that $\left.j_{1}^{-2}\right|_{\mathcal{C}_{2}}=-\operatorname{Id}_{\mathcal{C}_{2}}$ and $\left.j_{1}^{-2}\right|_{\mathcal{C}_{0}}=\operatorname{Id}_{\mathcal{C}_{0}}$. But since $j_{1} \in \operatorname{Aut}_{\otimes}\left(\operatorname{Id}_{\mathcal{B}}\right)$ we see that $j_{1}(X) \otimes j_{1}(Y)=j_{1}(X \otimes Y)=$ $\operatorname{id}_{X \otimes Y}$ for $X, Y \in \operatorname{Irr}\left(\mathcal{C}_{0}\right)$, and $j_{1}(X)=k \operatorname{id}_{X}$ and $j_{1}(Y)=k \operatorname{id}_{Y}$ since $j_{1}$ must act by a constant scalar on the simple objects in the universally-graded components. Thus $k= \pm 1$. But now $j_{1}^{-2}(X)=k^{2} \mathrm{id}_{X}=\mathrm{id}_{X}$, contradicting $\left.j_{1}^{-2}\right|_{\mathcal{C}_{2}}=-\mathrm{Id}_{\mathcal{C}_{2}}$. Alternatively we see that the second obstruction (4.14) implies that $(a, b) \mapsto \chi_{\lambda(a, b)}$ should define a coboundary $\mathbb{Z} / 2 \times \mathbb{Z} / 2 \rightarrow \widehat{\mathbb{Z} / 2}$. But in this case $(1,1) \mapsto \psi_{\mathbb{Z} / 2}$ where $\psi_{\mathbb{Z} / 2}(1)=-1$ is a non-trivial character, and hence the corresponding cocycle is nontrivial. We conclude that this associative zesting does not admit a braided zesting.

6.3.3. $S U(4)_{2}$. The metaplectic [6] $\mathbb{Z} / 4$-graded modular category $\mathcal{B}=S U(4)_{2} \cong$ $S O(6)_{2}$ has rank 10 and dimension 24 , with pointed subcategory $\mathcal{P}(4,2) \cong \mathcal{C}(\mathbb{Z} / 4, \eta)$ where $\eta(j)=e^{2 \pi i j^{2} / 4}$. As above we will denote the generator of $\operatorname{Inv}(\mathcal{B})$ by $g$ and in this case $h=g^{2}$ generates $\operatorname{Inv}\left(\mathcal{B}_{a d}\right)$. We set $q=e^{-2 \pi i / 4}$ so that the grading is of the form $\mathcal{B}_{0}=\left\{\mathbf{1}, g^{2}, Y_{1}\right\}, \mathcal{B}_{2}=\left\{g, g^{3}, Y_{2}\right\}, \mathcal{B}_{1}=\left\{X_{1}, X_{2}\right\}$ and $\mathcal{B}_{3}=\left\{Z_{1}, Z_{2}\right\}$ where $X_{1}$ is labeled by $\varpi_{1}$ and $Z_{i} \cong X_{i}^{*}$. The dimensions are $\operatorname{dim}\left(Y_{i}\right)=2$ and $\operatorname{dim}\left(X_{i}\right)=\operatorname{dim}\left(Z_{i}\right)=$ $\sqrt{3}$. We have two choices of zesting 2-cocycle $\lambda_{a} \in H^{2}(\mathbb{Z} / 4, \mathbb{Z} / 2) \cong \mathbb{Z}_{2}$ given by $\lambda_{a}(i, j)=h^{a}=g^{2 a}$ for $i+j \geq 4$ and 1 otherwise, where $a=0,1$. In the notation of Propositions 6.3 and 6.4 we have $m=2$ and $\epsilon=0$, and $N=4$ so that there are 8 associative zestings, taking $\lambda_{b} \in H^{3}\left(\mathbb{Z} / 4, \mathbb{k}^{\times}\right)$as in eqn. 6.13). By Proposition 6.4(i) the 4 pairs $\left(\lambda_{a}, \lambda_{b}\right)$ that admit braidings correspond to $\{(0,0),(0,2),(1,1),(1,3)\}$ so that we have at most 16 braided zestings, depending on $a, b$ and $s$ with $s^{4}=q^{-b}$. In fact, we have one such braided zesting for each $s=e^{2 \pi i x / 16}$, since each 16 th root of unity appears. Each of these braided zestings admits 2 ribbon twists, one of which is unitary. We will spare the reader the full details, but there are a few interesting things to note:

(1) When $a=0$ and $b=0,2$ the 8 unitary ribbon braided zestings are modular and in fact remain metaplectic [6]. Since zesting leaves the trivial component unchanged, and the 2 dimension object in the trivial component has twist $e^{2 \pi i / 3}$, we cannot obtain the complex conjugate category by zesting. Indeed, we can check directly that we get 2 distinct sets of twists among the 4 choices of $s^{4}=1$ (for $b=0$ ) and similarly for the 4 choices of $s^{4}=-1$ (for $b=2$ ). The central charges for $b=0$ are all the same, as are the central charges for 
$b=2$, and they are complex conjugates of each other (the cases $b=0$ and $b=2)$.

(2) For $a=1$ and $b=1,3$ we see that $g \otimes_{1} g=g \otimes g \otimes g^{2}=\mathbf{1}$, so that $g$ is self-dual in the zested theory $\mathcal{B}(1, b)$. Moreover, $s^{-4}=q^{b}= \pm i$ so that the twist of $g$ in $\mathcal{B}(1, b)$ is \pm 1 and is thus a boson or fermion. Lemma 5.8 show that, in fact $g$ is in the Müger center of $\mathcal{B}(1, b)$, so that $\mathcal{B}(1, b) \cong \operatorname{Rep}(\mathbb{Z} / 2, z) \otimes \mathcal{A}$ where either $z=0$ or $z=1$ and $\mathcal{A}$ is a Galois conjugate of $S U(2)_{4}$. In particular, $\mathcal{B}(1, b)$ is not modular.

\section{Conclusions and Future Directions}

We have developed the general theory of associative zesting for fusion categories and a further theory of braided, twist and ribbon zestings for categories with these additional structures and properties. We have illustrated their utility with a few examples, notably establishing the existence of a modular category of rank 10 and dimension 36 obtained by zesting $S U(3)_{3}$ that was conjectured in [5]. Moreover we have shown that braided zesting preserves property $F$, and given explicit computations of the modular data for braided zestings of modular categories. While zesting shares some similarities with symmetry gauging, the explicit nature of zesting is a distinct advantage.

This work suggests several interesting directions for future applications. Note that we have mostly applied our theory to zesting modular categories with respect to the universal grading. While these are perhaps the most interesting and most transparent examples, it would be interesting to apply associative zesting to fusion categories that do not admit a braiding and braided zesting with respect to non-universal grading groups and non-cyclic grading groups. Finally we point out that symmetry gauging has a physical interpretation as phase transitions of topological phases of matter. We do not know if zesting has a meaningful physical interpretation.

\section{REFERENCES}

[1] Bojko Bakalov and Alexander Kirillov, Jr. Lectures on tensor categories and modular functors, volume 21 of University Lecture Series. American Mathematical Society, Providence, RI, 2001.

[2] Nicolas Bourbaki. Lie groups and Lie algebras. Chapters 4-6. Elements of Mathematics (Berlin). Springer-Verlag, Berlin, 2002. Translated from the 1968 French original by Andrew Pressley.

[3] Alain Bruguières. Catégories prémodulaires, modularisations et invariants des variétés de dimension 3. Math. Ann., 316(2):215-236, 2000.

[4] Paul Bruillard, César Galindo, Tobias Hagge, Siu-Hung Ng, Julia Yael Plavnik, Eric C. Rowell, and Zhenghan Wang. Fermionic modular categories and the 16-fold way. J. Math. Phys., 58(4):041704, 31, 2017.

[5] Paul Bruillard, César Galindo, Seung-Moon Hong, Yevgenia Kashina, Deepak Naidu, Sonia Natale, Julia Yael Plavnik, and Eric C. Rowell. Classification of integral modular categories of Frobenius-Perron dimension $p q^{4}$ and $p^{2} q^{2}$. Canad. Math. Bull., 57(4):721-734, 2014.

[6] Paul Bruillard, Paul Gustafson, Julia Yael Plavnik, and Eric C Rowell. Dimension as a quantum statistic and the classification of metaplectic categories. Topological Phases of Matter and Quantum Computation, 747:89, 2020.

[7] Paul Bruillard, Siu-Hung Ng, Eric C. Rowell, and Zhenghan Wang. Rank-finiteness for modular categories. J. Amer. Math. Soc., 29(3):857-881, 2016. 
[8] Shawn X. Cui, César Galindo, Julia Yael Plavnik, and Zhenghan Wang. On Gauging Symmetry of Modular Categories. Comm. Math. Phys., 348(3):1043-1064, 2016.

[9] P. Deligne. Catégories tannakiennes. In The Grothendieck Festschrift, Vol. II, volume 87 of Progr. Math., pages 111-195. Birkhäuser Boston, Boston, MA, 1990.

[10] Vladimir Drinfeld, Shlomo Gelaki, Dmitri Nikshych, and Victor Ostrik. On braided fusion categories. I. Selecta Math. (N.S.), 16(1):1-119, 2010.

[11] Cain Edie-Michell. Auto-equivalences of the modular tensor categories of type $A, B, C$ and $G$. arXiv preprint arXiv:2002.03220, 2020.

[12] Samuel Eilenberg and Saunders Mac Lane. On the groups of $H(\Pi, n)$. I. Ann. of Math. (2), 58:55-106, 1953.

[13] Samuel Eilenberg and Saunders Mac Lane. On the groups $H(\Pi, n)$. II. Methods of computation. Ann. of Math. (2), 60:49-139, 1954.

[14] Pavel Etingof, Shlomo Gelaki, Dmitri Nikshych, and Victor Ostrik. Tensor categories, volume 205 of Mathematical Surveys and Monographs. American Mathematical Society, Providence, RI, 2015.

[15] Pavel Etingof, Dmitri Nikshych, and Victor Ostrik. Fusion categories and homotopy theory. Quantum Topol., 1(3):209-273, 2010. With an appendix by Ehud Meir.

[16] Pavel Etingof, Dmitri Nikshych, and Victor Ostrik. Weakly group-theoretical and solvable fusion categories. Adv. Math., 226(1):176-205, 2011.

[17] Shlomo Gelaki, Deepak Naidu, and Dmitri Nikshych. Centers of graded fusion categories. Algebra Number Theory, 3(8):959-990, 2009.

[18] Shlomo Gelaki and Dmitri Nikshych. Nilpotent fusion categories. Advances in Mathematics, 217(3):1053 - 1071, 2008.

[19] A Henriques, D Penneys, and J Tener. Categorified trace for module tensor categories over braided tensor categories. Documenta Mathematica, 21(2016):1089-1149, 2016.

[20] Gerhard Hochschild and Jean-Pierre Serre. Cohomology of group extensions. Transactions of the American Mathematical Society, pages 110-134, 1953.

[21] David Kazhdan and Hans Wenzl. Reconstructing monoidal categories. In I. M. Gelfand Seminar, volume 16 of Adv. Soviet Math., pages 111-136. Amer. Math. Soc., Providence, RI, 1993.

[22] Michaël Mignard and Peter Schauenburg. Modular categories are not determined by their modular data. arXiv preprint arXiv:1708.02796, 2017.

[23] Deepak Naidu and Eric C. Rowell. A finiteness property for braided fusion categories. Algebr. Represent. Theory, 14(5):837-855, 2011.

[24] Eric C. Rowell. From quantum groups to unitary modular tensor categories. In Representations of algebraic groups, quantum groups, and Lie algebras, volume 413 of Contemp. Math., pages 215-230. Amer. Math. Soc., Providence, RI, 2006.

[25] Cumrun Vafa. Toward classification of conformal theories. Physics Letters B, 206(3):421 - 426, 1988.

[26] Charles A. Weibel. An Introduction to Homological Algebra. Cambridge Studies in Advanced Mathematics. Cambridge University Press, 1994. 
Department of Mathematics, Indiana University

E-mail address: crdelane@iu.edu

Departamento de Matemáticas, Universidad de los Andes, Bogotá, Colombia

E-mail address: cn.galindo1116@uniandes.edu.co

Department of Mathematics, Indiana University

E-mail address: jplavnik@iu.edu

Department of Mathematics, Texas A\&M University

E-mail address: rowell@math.tamu.edu

Department of Mathematics, Texas A\&M University

E-mail address: zhangqing@math.tamu.edu 


\begin{tabular}{|c|c|c|c|}
\hline Label & Grading & Dimension & Twist \\
\hline 1 & 0 & 1 & 1 \\
\hline$g$ & 0 & 1 & -1 \\
\hline$g^{2}$ & 0 & 1 & 1 \\
\hline$g^{3}$ & 0 & 1 & -1 \\
\hline$Y$ & 0 & $2 d-1$ & -1 \\
\hline$g Y$ & 0 & $2 d-1$ & 1 \\
\hline$g^{2}$ & 0 & $2 d-1$ & -1 \\
\hline$g^{3} Y$ & 0 & $2 d-1$ & 1 \\
\hline$Z$ & 0 & $2 d-3$ & $-i$ \\
\hline$g Z$ & 0 & $2 d-3$ & $i$ \\
\hline$X$ & 1 & $\sqrt{2 d}$ & $\zeta_{64}^{15}$ \\
\hline$g X$ & 1 & $\sqrt{2 d}$ & $\zeta_{64}^{31}$ \\
\hline$g^{2} X$ & 1 & $\sqrt{2 d}$ & $-\zeta_{64}^{15}$ \\
\hline$g^{3} X$ & 1 & $\sqrt{2 d}$ & $-\zeta_{64}^{31}$ \\
\hline$\tilde{X}$ & 1 & $\sqrt{14 d-8}$ & $-\zeta_{64}^{7}$ \\
\hline$g \tilde{X}$ & 1 & $\sqrt{14 d-8}$ & $-\zeta_{64}^{23}$ \\
\hline$g^{2} \tilde{X}$ & 1 & $\sqrt{14 d-8}$ & $\zeta_{64}^{7}$ \\
\hline$g^{3} \tilde{X}$ & 1 & $\sqrt{14 d-8}$ & $\zeta_{64}^{23}$ \\
\hline$X^{\prime}$ & 2 & $d$ & $\zeta_{16}^{5}$ \\
\hline$g X^{\prime}$ & 2 & $d$ & $\zeta_{16}^{5}$ \\
\hline$g^{2} X^{\prime}$ & 2 & $d$ & $\zeta_{16}^{5}$ \\
\hline$g^{3} X^{\prime}$ & 2 & $d$ & $\zeta_{16}^{5}$ \\
\hline$X^{\prime \prime}$ & 2 & $d$ & $-\zeta_{16}$ \\
\hline$g X^{\prime \prime}$ & 2 & $d$ & $-\zeta_{16}$ \\
\hline$g^{2} X^{\prime \prime}$ & 2 & $d$ & $-\zeta_{16}$ \\
\hline$g^{3} X^{\prime \prime}$ & 2 & $d$ & $-\zeta_{16}$ \\
\hline$W$ & 2 & $4 d-4$ & $-\zeta_{16}^{7}$ \\
\hline$X^{*}$ & 3 & $\sqrt{2 d}$ & $\zeta_{64}^{15}$ \\
\hline$g X^{*}$ & 3 & $\sqrt{2 d}$ & $-\zeta_{64}^{31}$ \\
\hline$g^{2} X^{*}$ & 3 & $\sqrt{2 d}$ & $-\zeta_{64}^{15}$ \\
\hline$g^{3} X^{*}$ & 3 & $\sqrt{2 d}$ & $\zeta_{64}^{31}$ \\
\hline$\tilde{X}^{*}$ & 3 & $\sqrt{14 d-8}$ & $-\zeta_{64}^{7}$ \\
\hline$g \tilde{X}^{*}$ & 3 & $\sqrt{14 d-8}$ & $\zeta_{64}^{23}$ \\
\hline$g^{2} \tilde{X}^{*}$ & 3 & $\sqrt{14 d-8}$ & $\zeta_{64}^{7}$ \\
\hline$g^{3} \tilde{X}^{*}$ & 3 & $\sqrt{14 d-8}$ & $-\zeta_{64}^{23}$ \\
\hline
\end{tabular}

TABlE 2. Basic data for isomorphism classes of simple objects in $S U(4)_{4}$. Here $d=\sqrt{2}+2$ and $\zeta_{16}=e^{2 \pi i / 16}, \zeta_{64}=e^{2 \pi i / 64}$ are primitive 16 th and 64 th roots of unity, respectively. 